\title{
Indole Synthesis via Rhodium Catalyzed Oxidative Coupling of Acetanilides and Internal Alkynes
}

\author{
David R. Stuart, Mégan Bertrand-Laperle, Kevin M. N. Burgess, Keith Fagnou* \\ Center for Catalysis Research and Innovation, Department of Chemistry, University of \\ Ottawa, 10 Marie Curie, Ottawa, (CANADA) K1N 6N5 \\ keith.fagnou@science.uottawa.ca
}

\section{Supporting Information}

\section{General Methods:}

All rhodium catalyzed oxidative couplings of acetanilides and alkynes were carried out with no precautions to exclude oxygen or moisture. ${ }^{1} \mathrm{H}$ and ${ }^{13} \mathrm{C}$ NMR spectra were recorded in $\mathrm{CDCl}_{3}$ or DMSO- $d_{6}$ solutions on a Bruker AVANCE 300 or $400 \mathrm{MHz}$ spectrometer. High-resolution mass spectra were obtained on a Kratos Concept IIH. Infra-Red analysis was performed with a Bruker EQUINOX 55. HPLC Grade THF and $\mathrm{CH}_{2} \mathrm{Cl}_{2}$ are dried and purified via MBraun SP Series solvent purification system. Triethylamine was freshly distilled from $\mathrm{NaOH}$ before every use. $\left[\mathrm{Cp}^{*} \mathrm{RhCl}_{2}\right]_{2}$ was purchased from Strem and was stored in a dessicator and weighed out to air. Silver hexafluoroantimonate was purchased from Aldrich and stored and weighed out in a glove-box. All other reagents and solvents were used as is from commercial sources. Unless noted below, all other compounds have been reported in the literature or are commercially available.

\section{Table of Contents:}

Representative procedure for the preparation of acetanilides $\quad$ S2

$\begin{array}{ll}\text { Characterization data for acetanilides } & \text { S2 }\end{array}$

Procedures for the preparation of alkynes $\quad$ S5

Characterization data for alkynes $\quad$ S5

$\begin{array}{ll}\text { Representative procedure for preparation of indoles } & \text { S7 }\end{array}$

$\begin{array}{ll}\text { Characterization data for indoles } & \text { S7 }\end{array}$

Representative procedure for the deacetylation of $N$-acetylindoles $\quad$ S17

$\begin{array}{ll}\text { Characterization data for } N \text {-H-indoles } & \text { S17 }\end{array}$

$\begin{array}{ll}\text { Mechanistic Studies } & \text { S18 }\end{array}$

${ }^{1} \mathrm{H}$ and ${ }^{13} \mathrm{C}$ NMR spectra of all new compounds $\quad$ S23 


\section{Represenative procedure for the preparation of acetanilides:}

Aniline (10.1 mL, $109.7 \mathrm{mmol}, 1 \mathrm{eq})$ was added to a round-bottom flask via syringe and fitted with a rubber septum. The flask was purged with argon and dry DCM (300 mL, $0.4 \mathrm{M}$ ) was added. Acetic anhydride (12.5 mL, $132.2 \mathrm{mmol}, 1.2 \mathrm{eq}$ ) was added and the reaction was stirred at room temperature and monitored by TLC. Upon completion (generally a couple of hours, but as short as 20 minutes) the reaction mixture was washed with a saturated solution of sodium carbonate, the organic layers dried with $\mathrm{MgSO}_{4}$ and the solvent removed under reduced pressure. The product was obtained in quantitative yield (14.8 g). In most cases analytically pure acetanilides can be obtained after extraction however if necessary purification by flash chromatography with ethyl acetate/pet. ether was used (see below for specific conditions).

\section{Acetanilide (1a):}<smiles>CC(=O)Nc1ccccc1</smiles>

The above procedure was followed to afford the product in quantitative yield. This compound can also be purchased from commercial sources (CAS: 103-84-4).

${ }^{1}$ H NMR (400 MHz, $\mathbf{C D C l}_{3}, 293$ K): $\delta 7.50$ (d, $\left.J=7.8 \mathrm{~Hz}, 2 \mathrm{H}\right), 7.32$ (t, $J=7.9 \mathrm{~Hz}$, $2 \mathrm{H}), 7.10(\mathrm{t}, J=7.4 \mathrm{~Hz}, 1 \mathrm{H}), 2.17(\mathrm{~s}, 3 \mathrm{H})$. The signal for the exchangeable NH does not appear in the spectrum.

\section{4-Methoxyacetanilide (1b):}<smiles>COc1ccc(NC(C)=O)cc1</smiles>

The above procedure was followed to afford the product in $84 \%$ yield. This compound can also be purchased from commercial sources (CAS: 51-66-1).

${ }^{1}$ H NMR (400 MHz, $\mathbf{C D C l}_{3}, 293$ K): $\delta 7.44$ (brs, $1 \mathrm{H}$ ), 7.38 (d, $\left.J=8.8 \mathrm{~Hz}, 2 \mathrm{H}\right), 6.84$ (d, $J=9.2 \mathrm{~Hz}, 2 \mathrm{H}), 3.78$ (s, 3H), 2.14 (s, 3H).

\section{4-Fluoroacetanilide (1c):}<smiles>CC(=O)Nc1ccc(F)cc1</smiles>

The above procedure was followed to afford the product in $83 \%$ yield. This compound can also be purchased from commercial sources (CAS: 351-83-7). 
${ }^{1}$ H NMR (400 MHz, $\mathbf{C D C l}_{3}, 293$ K): $\delta 7.45$ (dd, $J=9.2 \mathrm{~Hz}, J=5.2 \mathrm{~Hz}, 2 \mathrm{H}$ ), 7.30 (brs, $1 \mathrm{H}), 7.01$ (brt, $J=8.8 \mathrm{~Hz}, 2 \mathrm{H}), 2.17$ (s, 3H).

Methyl 4-(acetylamino)benzoate (1d):<smiles>CC(=O)Nc1ccc(C(C)=O)cc1</smiles>

The above procedure was followed to afford the product in $83 \%$ yield. Spectral data was consistent with that previously reported. ${ }^{1}$

${ }^{1}$ H NMR (400 MHz, $\mathbf{C D C l}_{3}, 293$ K): $\delta 8.00$ (d, $\left.J=8.8 \mathrm{~Hz}, 2 \mathrm{H}\right), 7.59$ (d, $J=8.6 \mathrm{~Hz}$, 2H), 7.40 (brs, 1H), 3.90 (s, 3H), 2.11 (s, 3H).

\section{4-Chloroacetanilide (1e):}<smiles>CC(=O)Nc1ccc(Cl)cc1</smiles>

The above procedure was followed to afford the product in 51\% yield. This compound can also be purchased from commercial sources (CAS: 539-03-7).

${ }^{1}$ H NMR (400 MHz, $\mathbf{C D C l}_{3}, 293$ K): $\delta 7.46$ (d, $\left.J=8.8 \mathrm{~Hz}, 2 \mathrm{H}\right), 7.28$ (d, $J=8.4 \mathrm{~Hz}$, $2 \mathrm{H}), 2.17$ ( $\mathrm{s}, 3 \mathrm{H})$. The signal for the exchangeable $\mathrm{NH}$ does not appear in the spectrum.

\section{2-Methylacetanilide (1f):}<smiles>CC(=O)Nc1ccccc1C</smiles>

The above procedure was followed and column chromatography was performed on silica gel with ethyl acetate/pet. ether (70:30) as the solvent to afford the product in 73\% yield. This compound can also be purchase for commercial source (CAS: 120-66-1).

${ }^{1}$ H NMR (400 MHz, $\mathbf{C D C l}_{3}, 293$ K): $\delta 7.75$ (d, $\left.J=8.0 \mathrm{~Hz}, 1 \mathrm{H}\right), 7.22-7.17$ (m, 2H), 7.08 (t, $J=7.6 \mathrm{~Hz}, 1 \mathrm{H}), 7.01$ (brs, 1H), 2.26 (s, 3H), 2.20 (s, 3H).

\section{1-Naphthalenylacetanilide (1g):}<smiles>CC(=O)Nc1cccc2ccccc12</smiles> 
The above procedure was followed to afford the product in $50 \%$ yield. This compound can also be purchase for commercial source (CAS: 575-36-0).

${ }^{1}$ H NMR (300 MHz, DMSO-d $\mathbf{d}_{\mathbf{6}}, 293 \mathrm{~K}$ ): $\delta 9.93$ (br. s, 1H), 8.12 - 8.07 (m, 1H), 7.96 7.89 (m, 1H), 7.74 (d, $J=8.2 \mathrm{~Hz}, 1 \mathrm{H}), 7.70$ (d, $J=7.3 \mathrm{~Hz}, 1 \mathrm{H}), 7.58-7.46$ (m, 3H), 2.19 (s, 3H).

\section{3,5-Dimethoxyacetanilide (1h):}<smiles>COc1cc(NC(C)=O)cc(OC)c1</smiles>

The above procedure was followed to afford the product in $73 \%$ yield. Spectral data was consistent with that previously reported. ${ }^{2}$

${ }^{1}$ H NMR (400 MHz, CDCl 3 , 293 K): $\delta 7.23$ (brs, 1H), 6.75 (d, $J=2.0 \mathrm{~Hz}, 2 \mathrm{H}$ ), 6.23 (t, $J=1.6 \mathrm{~Hz}, 1 \mathrm{H}), 3.77$ (s, 6H), 2.16 (s, 3H).

\section{3-Methoxyacetanilide (1i)}<smiles>COc1cccc(NC(C)=O)c1</smiles>

The above procedure was followed to afford the product in $75 \%$ yield. This compound can also be purchased from commercial sources (CAS: 588-16-9).

${ }^{1}$ H NMR (400 MHz, CDCl M $_{3} 293$ K): $\delta 7.65$ (br. s, 1H), 7.27 (t, $J=2.0$ Hz, 1H), 7.19 (t, $J=8.0 \mathrm{~Hz}, 1 \mathrm{H}$ ), 6.98 (br. d, $J=8.0 \mathrm{~Hz}, 1 \mathrm{H}$ ), 6.65 (br. d, $J=8.0 \mathrm{~Hz}, 1 \mathrm{H}$ ), 3.77 (s, 3H), $2.15(\mathrm{~s}, 3 \mathrm{H})$.

\section{2-Naphthalenylacetanilide (1j):}<smiles>CC(=O)Nc1ccc2ccccc2c1</smiles>

The above procedure was followed to afford the product in $77 \%$ yield. This compound can also be purchase for commercial source (CAS: 581-97-5).

${ }^{1} \mathbf{H}$ NMR (300 MHz, $\left.\mathbf{C D C l}_{3}, 293 \mathbf{~ K )}\right) \delta 8.17$ (s, 1H), 7.92 (br., s, 1H), $7.76-7.67$ (m, 3H), $7.48-7.32$ (m, 3H), 2.19 (s, 3H). 


\section{Procedures for the preparation of alkynes:}

\section{1-Phenylpropyne (2a):}

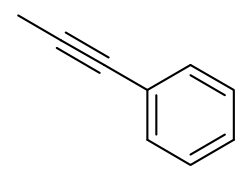

To a flame-dried round-bottom flask under argon was added phenylacetylene $(5 \mathrm{~mL}, 46$ mmol, $1 \mathrm{eq})$ followed by THF ( $300 \mathrm{~mL}, 0.15 \mathrm{M})$. The flask was placed in an icewater/salt bath and allowed to cool. $n$-Butyllithium $(40 \mathrm{~mL}, 2.5 \mathrm{M}$ in hexanes, 100 mmol, 2 eq) was added slowly and the reaction was allowed to stir for $\sim 1$ hour. Iodomethane (6 mL, $96.2 \mathrm{mmol}$, $2.1 \mathrm{eq}$ ) was added at $-20 \mathrm{C}$ and the reaction was allowed to stir at room temperature for $\sim 1$ hour. The reaction was quenched with a saturated solution of ammonium chloride and extracted with dichloromethane. The organics were dried over $\mathrm{MgSO}_{4}$ and the solvent removed under pressure. The residue was purified by Kugelrohr distillation to afford the product in quantitative yield. This compound can also be purchased from commercial sources (CAS: 673-32-5).

${ }^{1}$ H NMR (400 MHz, $\mathbf{C D C l}_{3}, 293$ K): $\delta 7.41-7.36$ (m, 2H), $7.30-7.23$ (m, 3H), 2.05 (s, 3H).

\section{1,2-diphenylacetylene (2b):}

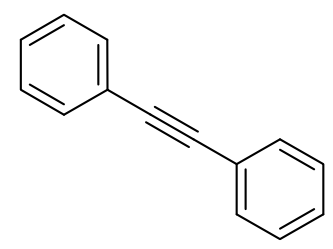

$\mathrm{Pd}\left(\mathrm{PPh}_{3}\right)_{2} \mathrm{Cl}_{2}$ (0.3429g, $\left.0.49 \mathrm{mmol}, 2 \mathrm{~mol} \%\right)$ and $\mathrm{CuI}$ (0.0925g, $\left.0.49 \mathrm{mmol}, 2 \mathrm{~mol} \%\right)$ were weighed out to a round bottom flask equipped with a magnetic stir bar and fitted with a rubber septa. The flask was purged with argon and THF (120 mL, $0.2 \mathrm{M})$ followed by triethylamine $(6 \mathrm{~mL})$ were added via syringe. Iodobenzene $(2.9 \mathrm{~mL}, 26$ mmol, $1.1 \mathrm{eq})$ followed by phenylacetylene $(2.7 \mathrm{~mL}, 25 \mathrm{mmol}, 1 \mathrm{eq})$ were added to the stirring mixture and the reaction was stirred overnight at room temperature. The volatiles were evaporated under reduced pressure and the residue was extracted with ethyl acetate/ether/brine. The organics were dried over $\mathrm{MgSO}_{4}$ and the solvent removed under reduced pressure. The residue was purified by flash column chromatography on silica gel with ether : pet. ether as the solvent to afford the title compound in $71 \%$ yield. This compound can also be purchased from commercial sources (CAS: 501-65-5).

${ }^{1}$ H NMR (400 MHz, CDCl 3 , 293 K): $\delta 7.56-7.51$ (m, 4H), 7.36 - 7.31 (m, 6H). 


\section{2-(Oct-1-ynyl)thiophene (2f):}

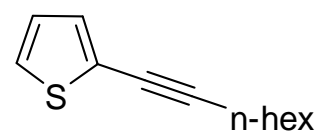

A mixture of 2-bromothiophene (126 uL, $1.3 \mathrm{mmol}, 1 \mathrm{eq}), 1$-octyne (148 uL, $1.6 \mathrm{mmol}$, $1.2 \mathrm{eq}$ ), pyrrolidine (217 uL, $2.6 \mathrm{mmol}, 2 \mathrm{eq}$ ), $\mathrm{PdCl}_{2}$ (4.6 mg, $0.03 \mathrm{mmol}, 2 \mathrm{~mol} \%$ ), $\mathrm{PPh}_{3}$ (13.6 mg, $0.05 \mathrm{mmol}, 4 \mathrm{~mol} \%)$, and degassed water $(2.6 \mathrm{~mL}, 0.5 \mathrm{M})$ under argon in a dried test tube was heated at $120{ }^{\circ} \mathrm{C}$ for 2 hours. It was then cooled and extracted with diethyl ether ( $3 \times 5 \mathrm{~mL}$ ). The organic layers were combined, washed with brine and dried with $\mathrm{MgSO}_{4}$. After evaporation under reduced pressure, the residue was purified by flash chromatography $(15 \mathrm{~cm} \times 3 \mathrm{~cm}$ ) with hexanes to afford a light yellow oil in $26 \%$ yield. Spectral data was consistent with previously reported data. ${ }^{3}$

${ }^{1}$ H NMR (300 MHz, $\mathbf{C D C l}_{3}, 293$ K): $\delta 7.16$ (dd, $J=5.1 \mathrm{~Hz}, 1.2 \mathrm{~Hz}, 1 \mathrm{H}$ ), 7.11 (dd, $J=$ $3.6 \mathrm{~Hz}, 0.9 \mathrm{~Hz}, 1 \mathrm{H}$ ), 6.93 (dd, $J=5.4 \mathrm{~Hz}, 3.6 \mathrm{~Hz}, 1 \mathrm{H}$ ), 2.42 (t, $J=7.2 \mathrm{~Hz}, 2 \mathrm{H}$ ), $1.64-$ $1.26(\mathrm{~m}, 8 \mathrm{H}), 0.90$ (t, $J=6.9 \mathrm{~Hz}, 3 \mathrm{H})$.

\section{$N$-Tosyl-5-bromoindole:}<smiles></smiles>

$\mathrm{NaH}$ (60\% suspension in paraffin) (210 mg, $5.3 \mathrm{mmol}, 1.05 \mathrm{eq})$ was added to a stirred solution of 5-bromoindole (980 mg, $5 \mathrm{mmol}, 1 \mathrm{eq})$ in anhydrous THF (10 mL, $0.5 \mathrm{M})$ at 0 ${ }^{\circ} \mathrm{C}$ over a 5 minute period. After stirring at room temperature for $1 \mathrm{~h}$, tosyl chloride (1.0 g, $5.3 \mathrm{mmol}, 1.05 \mathrm{eq}$ ) was added slowly. The reaction mixture was stirred for an additional 1 hour at room temperature and then poured into $50 \mathrm{~mL}$ of $5 \%$ aq. $\mathrm{NaHCO}_{3}$ and extracted with ether ( $3 \times 30 \mathrm{~mL}$ ). The combined organic layers were washed with brine, dried with $\mathrm{MgSO}_{4}$ and concentrated. Two recrystallizations from ethanol afforded the desired compound in 59\% yield. Spectral data was consistent with previously reported data. ${ }^{4}$

${ }^{1}$ H NMR (400 MHz, $\mathbf{C D C l}_{3}, 293$ K): $\delta 7.86(\mathrm{~d}, \mathrm{~J}=8.8 \mathrm{~Hz}, 1 \mathrm{H}), 7.74(\mathrm{~d}, \mathrm{~J}=8.4 \mathrm{~Hz}$, 2H), 7.66 (d, J = 1.8 Hz, 1H), 7.56 (d, J = 3.7 Hz, 1H), 7.40 (dd, J = 8.8 Hz, $1.9 \mathrm{~Hz}, 1 \mathrm{H}$ ), 7.23 (d, J = 8.1 Hz, 2H), 6.59 (d, J = 3.6 Hz, 1H), 2.35 (s, 3H).

\section{5-(Oct-1-ynyl)-1-tosylindole (2g):}

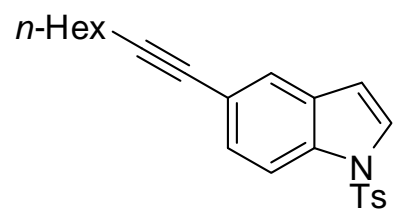


A mixture of 1-octyne (80 uL, $0.8 \mathrm{mmol}, 1.2 \mathrm{eq}), \mathrm{N}$-Tosyl-5-bromoindole (245 mg, 0.7 mmol, $1 \mathrm{eq})$ and $\mathrm{Pd}\left(\mathrm{PPh}_{3}\right)_{4}(40 \mathrm{mg}, 0.04 \mathrm{mmol}, 5 \mathrm{~mol} \%)$ in pyrrolidine $(1.8 \mathrm{~mL}, 0.4 \mathrm{M})$ under argon in a dried test tube were stirred at $50{ }^{\circ} \mathrm{C}$ overnight. The pyrrolidine was evaporated and the residue purified by column chromatography $(10 \mathrm{~cm}$ x $3 \mathrm{~cm}$ ) with $10 \%$ ether/hexanes to give $73 \%$ of the desired product.

${ }^{1}$ H NMR (400 MHz, CDCl 3 , 293 K): $\delta 7.89$ (d, $\left.J=8.6 \mathrm{~Hz}, 1 \mathrm{H}\right), 7.73$ (d, $J=8.4 \mathrm{~Hz}$, 2H), 7.56 (d, $J=1.0 \mathrm{~Hz}, 1 \mathrm{H}), 7.54$ (d, $J=3.7 \mathrm{~Hz}, 1 \mathrm{H}), 7.33$ (dd, $J=8.6 \mathrm{~Hz}, 1.5 \mathrm{~Hz}, 1 \mathrm{H}$ ), 7.21 (d, $J=8.0 \mathrm{~Hz}, 2 \mathrm{H}), 6.59$ (dd, $J=3.7 \mathrm{~Hz}, 0.7 \mathrm{~Hz}, 1 \mathrm{H}), 2.39$ (t, $J=7.1 \mathrm{~Hz}, 2 \mathrm{H}), 2.33$ (s, 3H), $1.63-1.56$ (m, 2H), $1.48-1.41$ (m, 2H), $1.34-1.30$ (m, 4H), 0.90 (t, $J=7.2$ $\mathrm{Hz}, 3 \mathrm{H})$.

${ }^{13}$ C NMR (100 MHz, $\mathbf{C D C l}_{3}, 293$ K): $\delta 145.1$ (C), 135.2 (C), 133.9 (C), 130.7 (C), $129.9(\mathrm{CH}), 128.1(\mathrm{CH}), 127.1(\mathrm{CH}), 127.8(\mathrm{CH}), 124.6(\mathrm{CH}), 119.2(\mathrm{C}), 113.4(\mathrm{CH})$, $108.9(\mathrm{CH}), 89.6(\mathrm{C}), 80.5(\mathrm{C}), 31.4\left(\mathrm{CH}_{2}\right), 28.8\left(\mathrm{CH}_{2}\right), 28.6\left(\mathrm{CH}_{2}\right), 22.6\left(\mathrm{CH}_{3}\right), 21.6$ $\left(\mathrm{CH}_{2}\right), 19.4\left(\mathrm{CH}_{2}\right), 14.1\left(\mathrm{CH}_{3}\right)$.

FTIR: 2930, 1457, 1375, 1175, $670 \mathrm{~cm}^{-1}$.

HRMS (EI): calculated for $\mathrm{C}_{23} \mathrm{H}_{25} \mathrm{NO}_{2} \mathrm{~S}\left(\mathrm{M}^{+}\right)$379.1606; found for $\mathrm{C}_{23} \mathrm{H}_{25} \mathrm{NO}_{2} \mathrm{~S}\left(\mathrm{M}^{+}\right)$ 379.1616.

$\mathbf{R}_{\mathbf{f}}$ (Ether/Hexanes (20:80)): 0.48 .

\section{Representative procedure for the preparation of indoles:}

To a 1 dram screw-cap vial was added $\operatorname{AgSbF}_{6}$ (0.0200 g, 0.06 mmol, 10 mol\%) in the glove-box. The vial was sealed and removed from the glove-box. $\left[\mathrm{Cp} * \mathrm{RhCl}_{2}\right]_{2}$ (0.0092 g, $0.015 \mathrm{mmol}, 2.5 \mathrm{~mol} \%)$, Cu(OAc) $)_{2} \cdot \mathrm{H}_{2} \mathrm{O}(0.252 \mathrm{~g}, 1.26 \mathrm{mmol}, 2.1 \mathrm{eq})$ and acetanilide ( $0.6 \mathrm{mmol}, 1 \mathrm{eq})$ are weighed into the vial open to air (if a solid, the alkyne is added at this point). $t$-AmOH $(3 \mathrm{~mL}, 0.2 \mathrm{M})$ and alkyne (0.66 mmol, $1.1 \mathrm{eq})$ if a liquid are added via syringe and the reaction is sealed and placed in a pre-heated $\left(120^{\circ} \mathrm{C}\right)$ block. The reaction is stirred for 1 hour (unless otherwise stated) and then cooled to room temperature and checked by TLC or GCMS. The reaction is filtered over Celite washing with ether into a $50 \mathrm{~mL}$ round-bottom flask and silica is added. The solvent is removed and the compound purified by flash column chromatography on silica gel $(10 \mathrm{~cm} \mathrm{x} 3 \mathrm{~cm})$ with ether/pet. ether as the solvent (see below for specific eluent composition).

\section{1-Acetyl-3-methyl-2-phenylindole (3a):}

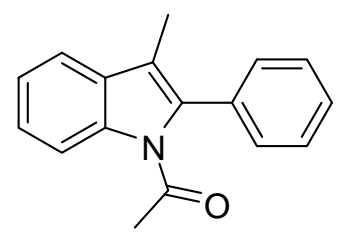

The above procedure was followed and column chromatography was performed on silica gel with ether/pet. ether (5:95) as the solvent to afford the product in $79 \%$ yield. In a separate experiment the above procedure was followed but the reaction time was reduced to 5 minutes and purification by column chromatography on silica gel with ether/pet. ether (5:95) as the solvent afforded the product in 65\% yield. Spectral data was consistent with that previously reported. ${ }^{5}$ 
${ }^{1}$ H NMR (400 MHz, CDCl, 293 K): $\delta 8.42$ (d, $\left.J=8.0 \mathrm{~Hz}, 1 \mathrm{H}\right), 7.55-7.30$ (m, 8H), 2.14 (s, 3H), 1.96 (s, 3H).

\section{1-Acetyl-5-methoxy-3-methyl-2-phenylindole (3b):}<smiles>COc1ccc2c(c1)c(C)c(-c1ccccc1)n2C(C)=O</smiles>

The above procedure was followed and column chromatography was performed on silica gel with ether/pet. ether (10:90) as the solvent to afford the product in $82 \%$ yield.

${ }^{1} \mathbf{H}$ NMR (400 MHz, $\mathbf{C D C l}_{3}$, $293 \mathrm{~K}$ ): $\delta 8.35$ (d, $\left.J=9.6 \mathrm{~Hz}, 1 \mathrm{H}\right), 7.51-7.41(\mathrm{~m}, 3 \mathrm{H})$, 7.38 (dd, $J=8.1 \mathrm{~Hz}, J=1.7 \mathrm{~Hz}, 2 \mathrm{H}$ ), 6.97 (dd, $J=8.6, J=2.6 \mathrm{~Hz}, 1 \mathrm{H}), 6.96$ (s, 1H), 3.89 (s, 3H), 2.10 (s, 3H), 1.93 (s, 3H).

${ }^{13}$ C NMR (100 MHz, $\mathbf{C D C l}_{3}, 293$ K): $\delta 170.0$ (C), 156.5 (C), 135.6 (C), 133.7 (C), 131.6 (C), $131.2(\mathrm{C}), 130.3(\mathrm{CH}), 128.7(\mathrm{CH}), 128.5(\mathrm{CH}), 118.0(\mathrm{C}), 117.4(\mathrm{CH}), 113.3$ $(\mathrm{CH}), 101.6(\mathrm{CH}), 55.8\left(\mathrm{CH}_{3}\right), 27.5\left(\mathrm{CH}_{3}\right), 9.3\left(\mathrm{CH}_{3}\right)$.

FTIR: 2834, 1652, 1371, 1229, $947 \mathrm{~cm}^{-1}$.

HRMS (EI): calculated for $\mathrm{C}_{18} \mathrm{H}_{17} \mathrm{NO}_{2}\left(\mathrm{M}^{+}\right)$279.1259; found for $\mathrm{C}_{18} \mathrm{H}_{17} \mathrm{NO}_{2}\left(\mathrm{M}^{+}\right)$ 279.1251.

Melting point (Ether/Pet. Ether): $92-93^{\circ} \mathrm{C}$.

$\mathbf{R}_{\mathbf{f}}($ Ether/Pet. Ether (10:90)): 0.19.

\section{1-Acetyl-5-fluoro-3-methyl-2-phenylindole (3c):}<smiles>CC(=O)n1c(-c2ccccc2)c(C)c2cc(F)ccc21</smiles>

The above procedure was followed with heating for 3 hours and column chromatography was performed on silica gel with ether/pet. ether (5:95) as the solvent to afford the product in $47 \%$ yield.

${ }^{1} \mathbf{H}$ NMR (400 MHz, $\left.\mathbf{C D C l}_{3}, 293 \mathrm{~K}\right): \delta 8.39$ (dd, $\left.J=9.1 \mathrm{~Hz}, J=4.7 \mathrm{~Hz}, 1 \mathrm{H}\right), 7.53-$ $7.43(\mathrm{~m}, 3 \mathrm{H}), 7.38$ (dd, $J=8.1 \mathrm{~Hz}, J=1.8 \mathrm{~Hz}, 2 \mathrm{H}), 7.16$ (dd, $J=8.6 \mathrm{~Hz}, J=2.6 \mathrm{~Hz}, 1 \mathrm{H}$ ), 7.08 (dd, $J=J=9.1 \mathrm{~Hz}, J=2.6 \mathrm{~Hz}, 1 \mathrm{H}$ ), 2.09 (s, 3H), 1.94 (s, 3H).

${ }^{13}$ C NMR (100 MHz, $\mathbf{C D C l}_{3}, 293$ K): $\delta 170.8$ (C), 159.7 (d, $J=240.3$, C), 136.5 (C), 133.3 (C), 133.1 (C), 131.4 (d, $J=9.3 \mathrm{~Hz}, \mathrm{C}$ ), $130.2(\mathrm{CH}), 128.8(\mathrm{CH}), 128.7$ (CH), 117.8 (d, $J=4.0 \mathrm{~Hz}, \mathrm{C}), 117.6$ (d, $J=8.8 \mathrm{~Hz}, \mathrm{CH}), 112.7$ (d, $J=24.4 \mathrm{~Hz}, \mathrm{CH}), 104.2$ (d, $\mathrm{J}=23.5 \mathrm{~Hz}, \mathrm{CH}), 27.5\left(\mathrm{CH}_{3}\right), 9.3\left(\mathrm{CH}_{3}\right)$.

FTIR: 1645, 1452, 1308, $1210 \mathrm{~cm}^{-1}$.

HRMS (EI): calculated for $\mathrm{C}_{17} \mathrm{H}_{14} \mathrm{FNO}\left(\mathrm{M}^{+}\right)$267.1059; found for $\mathrm{C}_{17} \mathrm{H}_{14} \mathrm{FNO}\left(\mathrm{M}^{+}\right)$ 267.1072. 
Melting point (Ether/Pet. Ether): $126-128^{\circ} \mathrm{C}$.

$\mathbf{R}_{\mathbf{f}}$ (Ether/Pet. Ether (5:95)): 0.21.

1-Acetyl-5-methylcarboxylate-3-methyl-2-phenylindole (3d):<smiles>CC(=O)c1ccc2c(c1)c(C)c(-c1ccccc1)n2C(C)=O</smiles>

The above procedure was followed and column chromatography was performed on silica gel with ether/pet. ether (10:90) as the solvent to afford the product in $69 \%$ yield.

${ }^{1} \mathrm{H}$ NMR (400 MHz, CDCl 3 , $\left.293 \mathrm{~K}\right): \delta 8.42(\mathrm{~d}, J=8.8 \mathrm{~Hz}, 1 \mathrm{H}), 8.26(\mathrm{~d}, J=2.4 \mathrm{~Hz}$, $1 \mathrm{H}$ ), 8.06 (dd, $J=8.8 \mathrm{~Hz}, J=1.7 \mathrm{~Hz}, 1 \mathrm{H}$ ), $7.54-7.39$ (m, 5H), 3.96 (s, 3H), 2.18 (s, $3 \mathrm{H}), 1.97$ (s, 3H).

${ }^{13}$ C NMR (100 MHz, CDCl, 293 K): $\delta 171.2$ (C), 167.5 (C), 139.4 (C), 136.1 (C), 133.0 (C), $130.2(\mathrm{CH}), 130.1(\mathrm{C}), 128.9(\mathrm{CH}), 128.8(\mathrm{CH}), 126.6(\mathrm{CH}), 125.3(\mathrm{C}), 120.9$ $(\mathrm{CH}), 118.4(\mathrm{C}), 115.9(\mathrm{CH}), 52.1\left(\mathrm{CH}_{3}\right), 27.7\left(\mathrm{CH}_{3}\right), 9.2\left(\mathrm{CH}_{3}\right)$.

FTIR: 2850, 1711, 1303, $1250 \mathrm{~cm}^{-1}$.

HRMS (EI): calculated for $\mathrm{C}_{19} \mathrm{H}_{17} \mathrm{NO}_{3}\left(\mathrm{M}^{+}\right)$307.1208; found for $\mathrm{C}_{19} \mathrm{H}_{17} \mathrm{NO}_{3}\left(\mathrm{M}^{+}\right)$ 307.1240.

Melting point (Ether/Pet. Ether): $112-114^{\circ} \mathrm{C}$.

$\mathbf{R}_{\mathbf{f}}($ Ether/Pet. Ether (10:90)): 0.21.

1-Acetyl-5-chloro-3-methyl-2-phenylindole (3e):<smiles>CC(=O)n1c(-c2ccccc2)c(C)c2cc(Cl)ccc21</smiles>

The above procedure was followed and column chromatography was performed on silica gel with ether/pet. ether (5:95) as the solvent to afford the product in $62 \%$ yield.

${ }^{1} \mathbf{H}$ NMR (400 MHz, $\left.\mathbf{C D C l}_{3}, 293 \mathrm{~K}\right): \delta 8.35$ (d, $\left.J=8.8 \mathrm{~Hz}, 1 \mathrm{H}\right), 7.52-7.30(\mathrm{~m}, 7 \mathrm{H})$, 2.10 (s, 3H), 1.94 (s, 3H).

${ }^{13}$ C NMR (100 MHz, CDCl $_{3}, 293$ K): $\delta 170.9$ (C), 136.2 (C), 135.1 (C), 133.1 (C), $131.6(\mathrm{C}), 130.2(\mathrm{CH}), 129.0(\mathrm{C}), 128.8(\mathrm{CH}), 125.3(\mathrm{CH}), 118.3(\mathrm{CH}), 117.5(\mathrm{CH})$, $117.4(\mathrm{C}), 27.6\left(\mathrm{CH}_{3}\right), 9.2\left(\mathrm{CH}_{3}\right)$. There is one overlapping carbon signal as 1 peak is missing even after prolonged scans.

FTIR: 2923, 1700, 1450, 1306, $706 \mathrm{~cm}^{-1}$.

HRMS (EI): calculated for $\mathrm{C}_{17} \mathrm{H}_{14} \mathrm{ClNO}\left(\mathrm{M}^{+}\right)$283.0764; found for $\mathrm{C}_{17} \mathrm{H}_{14} \mathrm{ClNO}\left(\mathrm{M}^{+}\right)$ 283.0748.

Melting point (Ether/Pet, Ether): $65-66^{\circ} \mathrm{C}$.

$\mathbf{R}_{\mathrm{f}}$ (Ether/Pet. Ether (5:95)): 0.28. 


\section{1-Acetyl-7-methyl-3-methyl-2-phenylindole (3f):}<smiles>CC(=O)n1c(-c2ccccc2)c(C)c2cccc(C)c21</smiles>

The above procedure was followed and column chromatography was performed on silica gel with ether/pet. ether (5:95) as the solvent to afford the product in $66 \%$ yield.

${ }^{1}$ H NMR (400 MHz, $\left.\mathbf{C D C l}_{3}, 293 \mathbf{~ K}\right): \delta 7.49-7.38$ (m, 6H), 7.22 (dd, $J=8.2 \mathrm{~Hz}, J=$ $6.8 \mathrm{~Hz}, 1 \mathrm{H}), 7.14$ (d, $J=7.0 \mathrm{~Hz}, 1 \mathrm{H}), 2.41$ (s, 3H), 2.13 (s, 3H), 1.99 (s, 3H).

${ }^{13}$ C NMR (100 MHz, $\mathbf{C D C l}_{3}, 293$ K): $\delta 172.1$ (C), 135.8 (C), 135.1 (C), 133.2 (C), $131.4(\mathrm{C}), 130.3(\mathrm{CH}), 128.6(\mathrm{CH}), 128.5(\mathrm{CH}), 127.6(\mathrm{CH}), 125.2(\mathrm{C}), 123.0(\mathrm{CH})$, $116.5(\mathrm{CH}), 116.2(\mathrm{C}), 28.2\left(\mathrm{CH}_{3}\right), 21.6\left(\mathrm{CH}_{3}\right), 9.2\left(\mathrm{CH}_{3}\right)$.

FTIR: 2926, 1714, 1303, 1198, $703 \mathrm{~cm}^{-1}$.

HRMS (EI): calculated for $\mathrm{C}_{18} \mathrm{H}_{17} \mathrm{NO}\left(\mathrm{M}^{+}\right)$263.1310; found for $\mathrm{C}_{18} \mathrm{H}_{17} \mathrm{NO}\left(\mathrm{M}^{+}\right)$ 263.1312.

$\mathbf{R}_{\mathbf{f}}$ (Ether/Pet. Ether (10:90)): 0.29.

1-Acetyl-3-methyl-2-phenylbenzo[g]indole (3g):<smiles>CC(=O)n1c(-c2ccccc2)c(C)c2ccc3ccccc3c21</smiles>

The above procedure was followed and column chromatography was performed on silica gel with ether/pet. ether (10:90) as the solvent to afford the product in $72 \%$ yield.

${ }^{1} \mathbf{H}$ NMR (400 MHz, $\left.\mathbf{C D C l}_{3}, 293 \mathrm{~K}\right): \delta 8.16$ (d, $\left.J=9.1 \mathrm{~Hz}, 1 \mathrm{H}\right), 7.93$ (d, $J=6.6 \mathrm{~Hz}$, $1 \mathrm{H}), 7.72$ (d, $J=8.5 \mathrm{~Hz}, 1 \mathrm{H}), 7.64$ (d, $J=8.5 \mathrm{~Hz}, 1 \mathrm{H}), 7.52-7.41$ (m, 7H), 2.25 (s, 3H), 2.19 (s, 3H).

${ }^{13}$ C NMR (100 MHz, $\mathbf{C D C l}_{3}, 293$ K): $\delta 175.2$ (C), 135.2 (C), 132.8 (C), 132.3 (C), 130.7 (C), $130.5(\mathrm{CH}), 130.1(\mathrm{C}), 129.2(\mathrm{CH}), 128.6(\mathrm{CH}), 128.4(\mathrm{CH}), 128.0(\mathrm{C}), 125.6$ $(\mathrm{CH}), 124.6(\mathrm{CH}), 124.3(\mathrm{CH}), 123.1(\mathrm{C}), 122.8(\mathrm{CH}), 118.1(\mathrm{CH}), 29.0\left(\mathrm{CH}_{3}\right), 9.3$ $\left(\mathrm{CH}_{3}\right)$.

FTIR: 2919, 1721, 1376, 1298, $808 \mathrm{~cm}^{-1}$.

HRMS (EI): calculated for $\mathrm{C}_{21} \mathrm{H}_{17} \mathrm{NO}\left(\mathrm{M}^{+}\right)$299.1310; found for $\mathrm{C}_{21} \mathrm{H}_{17} \mathrm{NO}\left(\mathrm{M}^{+}\right)$ 299.1322.

Melting point (Ether/Pet. Ether): $86-88^{\circ} \mathrm{C}$.

$\mathbf{R}_{\mathbf{f}}$ (Ether/Pet. Ether (10:90)): 0.35. 
1-Acetyl-4,6-dimethoxy-3-methyl-2-phenylindole (3h):<smiles>COc1cc(OC)c2c(C)c(-c3ccccc3)n(C(C)=O)c2c1</smiles>

The above procedure was followed and column chromatography was performed on silica gel with ether/pet. ether $(15: 85)$ as the solvent to afford the product as the major isomer in the reaction of 3,5-dimethoxyacetanilide with 3-phenyl-2-propyne in 70\% yield.

${ }^{1}$ H NMR (400 MHz, $\left.\mathbf{C D C l}_{3}, 293 \mathbf{~ K}\right): \delta 7.65$ (d, $\left.J=2.0 \mathrm{~Hz}, 1 \mathrm{H}\right), 7.46$ (dd, $J=J=7.4$ $\mathrm{Hz}, 2 \mathrm{H}$ ), 7.39 (tt, $J=7.3 \mathrm{~Hz}, J=1.5 \mathrm{~Hz}, 1 \mathrm{H}$ ), 7.34 (dd, $J=8.3 \mathrm{~Hz}, J=1.6 \mathrm{~Hz}, 2 \mathrm{H}), 6.38$ (d, $J=2.0 \mathrm{~Hz}, 1 \mathrm{H}), 3.89$ (s, 3H), 3.88 (s, 3H), 2.25 (s, 3H), 1.91 (s, 3H).

${ }^{13}$ C NMR (100 MHz, $\mathbf{C D C l}_{3}, 293$ K): $\delta 171.7$ (C), 159.5 (C), 154.7 (C), 138.7 (C), $133.9(\mathrm{C}), 132.0(\mathrm{C}), 130.5(\mathrm{CH}), 128.6(\mathrm{CH}), 128.0(\mathrm{CH}), 118.4(\mathrm{C}), 113.7$ (C), 95.3 $(\mathrm{CH}), 92.3(\mathrm{CH}), 55.8\left(\mathrm{CH}_{3}\right), 55.4\left(\mathrm{CH}_{3}\right), 27.9\left(\mathrm{CH}_{3}\right), 11.7\left(\mathrm{CH}_{3}\right)$.

FTIR: 1647, 1421, 1285, $1043 \mathrm{~cm}^{-1}$.

HRMS (EI): calculated for $\mathrm{C}_{19} \mathrm{H}_{19} \mathrm{NO}_{3}\left(\mathrm{M}^{+}\right)$309.1365; found for $\mathrm{C}_{19} \mathrm{H}_{19} \mathrm{NO}_{3}\left(\mathrm{M}^{+}\right)$ 309.1374.

Melting point (Ether/Pet. Ether): $103-104^{\circ} \mathrm{C}$.

$\mathbf{R}_{\mathbf{f}}$ (Ether/Pet. Ether (15:85)): 0.37.

\section{1-Acetyl-6-methoxy-3-methyl-2-phenylindole (3i-6-OMe):}<smiles>COc1ccc2c(C)c(-c3ccccc3)n(C(C)=O)c2c1</smiles>

The above procedure was followed and column chromatography was performed on silica gel with ether/pet. ether (10:90) as the solvent to afford the product as the major isomer in the reaction of 3-methoxyacetanilide with 3-phenyl-2-propyne in 73\% yield.

${ }^{1}$ H NMR (400 MHz, $\mathbf{C D C l}_{3}, 293$ K): $\delta 8.05$ (d, $\left.J=2.3 \mathrm{~Hz}, 1 \mathrm{H}\right), 7.50-7.35$ (m, 6H), 6.96 (dd, $J=8.5 \mathrm{~Hz}, J=2.4 \mathrm{~Hz}, 1 \mathrm{H}$ ), 3.90 (s, 3H), 2.12 (s, 3H), 1.94 (s, 3H).

${ }^{13}$ C NMR (100 MHz, $\mathbf{C D C l}_{3}, 293$ K): $\delta 171.4$ (C), 158.7 (C), 137.8 (C), 133.8 (C), $133.6(\mathrm{C}), 130.2(\mathrm{CH}), 128.7(\mathrm{CH}), 128.2(\mathrm{CH}), 124.2(\mathrm{C}), 119.0(\mathrm{CH}), 118.2(\mathrm{C}), 112.6$ $(\mathrm{CH}), 100.6(\mathrm{CH}), 55.8\left(\mathrm{CH}_{3}\right), 27.7\left(\mathrm{CH}_{3}\right), 9.3\left(\mathrm{CH}_{3}\right)$.

FTIR: 2936, 1695, 1484, 1320, $776 \mathrm{~cm}^{-1}$. 
HRMS (EI): calculated for $\mathrm{C}_{18} \mathrm{H}_{17} \mathrm{NO}_{2}\left(\mathrm{M}^{+}\right)$279.1259; found for $\mathrm{C}_{18} \mathrm{H}_{17} \mathrm{NO}_{2}\left(\mathrm{M}^{+}\right)$ 279.1254 .

Melting point (Ether/Pet. Ether): $76-78^{\circ} \mathrm{C}$.

$\mathbf{R}_{\mathbf{f}}($ Ether/Pet. Ether (10:90)): 0.21.

1-Acetyl-4-methoxy-3-methyl-2-phenylindole (3i-4-OMe):<smiles>COc1cccc2c1c(C)c(-c1ccccc1)n2C(C)=O</smiles>

The above procedure was followed and column chromatography was performed on silica gel with ether/pet. ether (10:90) as the solvent to afford the product as the minor isomer in the reaction of 3-methoxyacetanilide with 3-phenyl-2-propyne in 9\% yield.

${ }^{1} \mathbf{H}$ NMR (400 MHz, $\mathbf{C D C l}_{3}$, $\left.293 \mathrm{~K}\right): \delta 8.02(\mathrm{dd}, J=8.4 \mathrm{~Hz}, J=0.7 \mathrm{~Hz}, 1 \mathrm{H}), 7.50-$ 7.39 (m, 3H), 7.36 (dd, $J=8.2 \mathrm{~Hz}, J=1.7 \mathrm{~Hz}, 2 \mathrm{H}$ ), 7.26 (t, $J=8.2 \mathrm{~Hz}, 1 \mathrm{H}), 6.73$ (d, $J=$ $8.0 \mathrm{~Hz}, 1 \mathrm{H}), 3.92$ (s, 3H), 2.28 (s, 3H), 1.93 (s, 3H).

${ }^{13}$ C NMR (100 MHz, CDCl $_{3}, 293$ K): $\delta 171.3$ (C), 154.4 (C), 138.2 (C), 133.8 (C), 133.4 (C), 130.5 (CH), 128.7 (CH), 128.3 (CH), 126.0 (CH), 119.5 (C), 118.3 (C), 109.2 (CH), $104.4(\mathrm{CH}), 55.4\left(\mathrm{CH}_{3}\right), 27.8\left(\mathrm{CH}_{3}\right), 11.8\left(\mathrm{CH}_{3}\right)$.

FTIR: 1645, 1432, 1309, $1265 \mathrm{~cm}^{-1}$.

HRMS (EI): calculated for $\mathrm{C}_{18} \mathrm{H}_{17} \mathrm{NO}_{2}\left(\mathrm{M}^{+}\right)$279.1259; found for $\mathrm{C}_{18} \mathrm{H}_{17} \mathrm{NO}_{2}\left(\mathrm{M}^{+}\right)$ 279.1268.

Melting point (Ether/Pet. Ether): $82-84^{\circ} \mathrm{C}$.

$\mathbf{R}_{\mathbf{f}}($ Ether/Pet. Ether (10:90)): 0.31.

\section{1-Acetyl-3-methyl-2-phenylbenzo[f]indole (3j):}<smiles>CC(=O)n1c(-c2ccccc2)c(C)c2cc3ccccc3cc21</smiles>

The above procedure was followed and column chromatography was performed on silica gel with ether/pet. ether (10:90) as the solvent to afford the product in $62 \%$ yield.

${ }^{1} \mathbf{H}$ NMR (400 MHz, CDCl $_{3}$, 293 K): $\delta 8.96$ (s, 1H), 8.03 - 7.99 (m, 1H), $7.97-7.92$ (m, 1H), 7.92 (s, 1H), $7.54-7.41$ (m, 7H), 2.21 (s, 3H), 1.97 (s, 3H).

${ }^{13}$ C NMR (100 MHz, CDCl $_{3}, 293$ K): $\delta 170.7$ (C), 137.6 (C), 136.4 (C), 133.5 (C), 132.4 (C), $131.2(\mathrm{C}), 130.7$ (C), $130.1(\mathrm{CH}), 128.9(\mathrm{CH}), 128.8(\mathrm{CH}), 128.7(\mathrm{CH}), 127.8$ (CH), $124.8(\mathrm{CH}), 124.6(\mathrm{CH}), 118.4(\mathrm{C}), 116.3(\mathrm{CH}), 113.8(\mathrm{CH}), 27.6\left(\mathrm{CH}_{3}\right), 9.5$ $\left(\mathrm{CH}_{3}\right)$.

FTIR: $3056,1689,1318,871,746 \mathrm{~cm}^{-1}$. 
HRMS (EI): calculated for $\mathrm{C}_{21} \mathrm{H}_{17} \mathrm{NO}\left(\mathrm{M}^{+}\right)$299.1310; found for $\mathrm{C}_{21} \mathrm{H}_{17} \mathrm{NO}\left(\mathrm{M}^{+}\right)$ 299.1325.

Melting point (Ether/Pet. Ether): $121-123^{\circ} \mathrm{C}$.

$\mathbf{R}_{\mathbf{f}}($ Ether/Pet. Ether (10:90)): 0.21.

\section{1-Acetyl-2,3-diphenylindole (3k):}<smiles>CC(=O)n1c(-c2ccccc2)c(-c2ccccc2)c2ccccc21</smiles>

The above procedure was followed and column chromatography was performed on silica gel with ether/pet. ether (5:95) as the solvent to afford the product in $81 \%$ yield.

${ }^{1} \mathbf{H}$ NMR (400 MHz, CDCl 3 , $\left.293 \mathrm{~K}\right): \delta 8.46(\mathrm{~d}, J=8.5 \mathrm{~Hz}, 1 \mathrm{H}), 7.56(\mathrm{~d}, J=8.2 \mathrm{~Hz}$, $1 \mathrm{H}), 7.44-7.19(\mathrm{~m}, 12 \mathrm{H}), 2.00$ (s, 3H).

${ }^{13}$ C NMR (100 MHz, CDCl $_{3}, 293$ K): $\delta 171.6$ (C), 136.8 (C), 135.0 (C), 133.1 (C), $133.0(\mathrm{C}), 130.8(\mathrm{CH}), 130.4(\mathrm{CH}), 129.3(\mathrm{C}), 128.6(\mathrm{CH}), 128.6(\mathrm{CH}), 128.2(\mathrm{CH})$, $126.9(\mathrm{CH}), 125.5(\mathrm{CH}), 123.8(\mathrm{CH}), 123.4(\mathrm{C}), 119.6(\mathrm{CH}), 116.2(\mathrm{CH}), 27.9\left(\mathrm{CH}_{3}\right)$.

FTIR: 1650, 1443, 1313, $929 \mathrm{~cm}^{-1}$.

HRMS (EI): calculated for $\mathrm{C}_{22} \mathrm{H}_{17} \mathrm{NO}\left(\mathrm{M}^{+}\right)$311.1310; found for $\mathrm{C}_{22} \mathrm{H}_{17} \mathrm{NO}\left(\mathrm{M}^{+}\right)$ 311.1330.

Melting point (Ether/Pet. Ether): $123-124^{\circ} \mathrm{C}$.

$\mathbf{R}_{\mathbf{f}}($ Ether/Pet. Ether (5:95)): 0.21.

1-Acetyl-2,3-di-n-propylindole (3l):<smiles>CCCc1c(CCC)n(C(C)=O)c2ccccc12</smiles>

The above procedure was followed and column chromatography was performed on silica gel with ether/pet. ether (5:95) as the solvent to afford the product in 68\% yield. Spectral data was consistent with previously reported data. ${ }^{3}$

${ }^{1}$ H NMR (400 MHz, $\mathbf{C D C l}_{3}, 293$ K): $\delta 7.77-7.73(\mathrm{~m}, 1 \mathrm{H}), 7.51-7.46(\mathrm{~m}, 1 \mathrm{H}), 7.27$ $7.21(\mathrm{~m}, 2 \mathrm{H}), 2.97(\mathrm{t}, J=7.7 \mathrm{~Hz}, 2 \mathrm{H}), 2.76(\mathrm{~s}, 3 \mathrm{H}) 2.63(\mathrm{t}, \mathrm{J}=7.6 \mathrm{~Hz}, 2 \mathrm{H}), 1.68-1.56$ (m, 4H), $1.03-0.96$ (m, 6H). 


\section{1-Acetyl-2-hexyl-3-methylindole (3m-major isomer):}

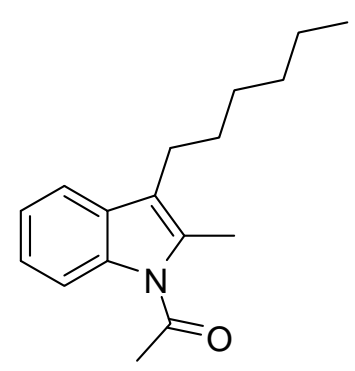

The above procedure was followed and column chromatography was performed on silica gel with ether/pet. ether (5:95) as the solvent to afford the product in 50\% yield.

${ }^{1}$ H NMR (400 MHz, CDCl $_{3}, 293$ K): $\delta 7.97$ - 7.94 (m, 1H), 7.47 - 7.45 (m, 1H), 7.27 $7.21(\mathrm{~m}, 2 \mathrm{H}), 2.72(\mathrm{~s}, 3 \mathrm{H}), 2.64(\mathrm{t}, J=7.6 \mathrm{~Hz}, 2 \mathrm{H}), 2.55$ (s, 3H), $1.61-1.54(\mathrm{~m}, 2 \mathrm{H})$, $1.38-1.28(\mathrm{~m}, 6 \mathrm{H}), 0.90-0.86(\mathrm{~m}, 3 \mathrm{H})$.

${ }^{13}$ C NMR (100 MHz, $\mathbf{C D C l}_{3}, 293$ K): $\delta 170.3$ (C), 135.8 (C), 132.6 (C), 130.7 (C), $123.6(\mathrm{CH}), 122.8(\mathrm{CH}), 120.4(\mathrm{C}), 118.3(\mathrm{CH}), 115.0(\mathrm{CH}), 31.8\left(\mathrm{CH}_{2}\right), 30.0\left(\mathrm{CH}_{2}\right)$, $29.3\left(\mathrm{CH}_{2}\right), 27.6\left(\mathrm{CH}_{3}\right), 23.9\left(\mathrm{CH}_{2}\right), 22.7\left(\mathrm{CH}_{2}\right), 14.4\left(\mathrm{CH}_{3}\right), 14.1\left(\mathrm{CH}_{3}\right)$.

FTIR: 2929, 1700, 1311, $746 \mathrm{~cm}^{-1}$.

HRMS (EI): calculated for $\mathrm{C}_{17} \mathrm{H}_{23} \mathrm{NO}\left(\mathrm{M}^{+}\right)$257.1780; found for $\mathrm{C}_{17} \mathrm{H}_{23} \mathrm{NO}\left(\mathrm{M}^{+}\right)$ 257.1877.

$\mathbf{R}_{\mathbf{f}}$ (Ether/Pet. Ether (5:95)): 0.31.

1-Acetyl-3-hexyl-2-methylindole (3m-minor isomer):

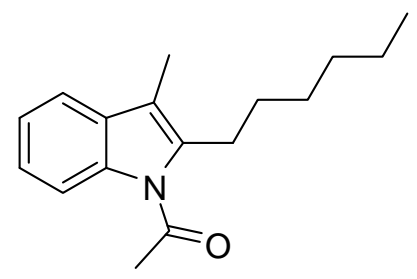

The above procedure was followed and column chromatography was performed on silica gel with ether/pet. ether (5:95) as the solvent to afford the product in $28 \%$ yield.

${ }^{1}$ H NMR (400 MHz, $\mathbf{C D C l}_{3}, 293$ K): $\delta 7.78$ - 7.74 (m, 1H), 7.47 - 7.43 (m, 1H), 7.27 $7.22(\mathrm{~m}, 2 \mathrm{H}), 3.00$ (t, $J=7.6 \mathrm{~Hz}, 2 \mathrm{H}), 2.76$ (s, 3H), 2.20 (s, 3H), $1.62-1.54(\mathrm{~m}, 2 \mathrm{H})$, $1.38-1.28(\mathrm{~m}, 6 \mathrm{H}), 0.90-0.86(\mathrm{~m}, 3 \mathrm{H})$.

${ }^{13}$ C NMR (100 MHz, CDCl 3 , 293 K): $\delta 170.0$ (C), 138.3 (C), 135.5 (C), 131.5 (C), $123.5(\mathrm{CH}), 122.7(\mathrm{CH}), 118.5(\mathrm{CH}), 115.3(\mathrm{C}), 114.5(\mathrm{CH}), 31.7\left(\mathrm{CH}_{2}\right), 29.9\left(\mathrm{CH}_{2}\right)$, $29.3\left(\mathrm{CH}_{2}\right), 27.7\left(\mathrm{CH}_{3}\right), 27.1\left(\mathrm{CH}_{2}\right), 22.7\left(\mathrm{CH}_{2}\right), 14.1\left(\mathrm{CH}_{3}\right), 8.7\left(\mathrm{CH}_{3}\right)$.

FTIR: 2926, 1644, 1317, $750 \mathrm{~cm}^{-1}$.

HRMS (EI): calculated for $\mathrm{C}_{17} \mathrm{H}_{23} \mathrm{NO}\left(\mathrm{M}^{+}\right)$257.1780; found for $\mathrm{C}_{17} \mathrm{H}_{23} \mathrm{NO}\left(\mathrm{M}^{+}\right)$ 257.1788.

$\mathbf{R}_{\mathbf{f}}$ (Ether/Pet. Ether (5:95)): 0.49 . 


\section{1-Acetyl-2-phenyl-3-n-propylindole (3n):}

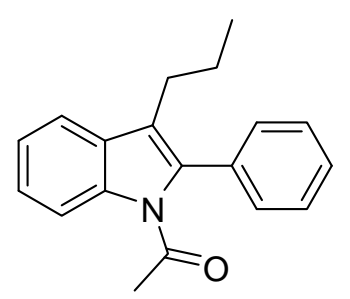

The above procedure was followed and column chromatography was performed on silica gel with ether/pet. ether (5:95) as the solvent to afford the product in $84 \%$ yield.

${ }^{1}$ H NMR (400 MHz, $\mathbf{C D C l}_{3}, 293$ K): $\delta 8.44$ (br. d, $J=8.0 \mathrm{~Hz}, 1 \mathrm{H}$ ), 7.56 (br. d, $J=7.2$ $\mathrm{Hz}, 1 \mathrm{H}), 7.50-7.28$ (m, 7H), 2.51 (t, $J=7.6 \mathrm{~Hz}, 2 \mathrm{H}), 1.93$ (s, 3H), $1.66-1.56$ (m, 2H), $0.86(\mathrm{t}, J=7.4 \mathrm{~Hz}, 3 \mathrm{H})$.

${ }^{13}$ C NMR (100 MHz, $\mathbf{C D C l}_{3}, 293$ K): $\delta 171.1$ (C), 136.9 (C), 134.9 (C), 133.7 (C), $130.4(\mathrm{CH}), 129.6(\mathrm{C}), 128.7(\mathrm{CH}), 128.6(\mathrm{CH}), 125.2(\mathrm{CH}), 123.4(\mathrm{CH}), 122.8(\mathrm{C})$, $118.9(\mathrm{CH}), 116.5(\mathrm{CH}), 27.7\left(\mathrm{CH}_{2}\right), 26.4\left(\mathrm{CH}_{3}\right), 23.4\left(\mathrm{CH}_{3}\right), 14.2\left(\mathrm{CH}_{2}\right)$.

FTIR: 2959, 1698, 1453, 1306, $749 \mathrm{~cm}^{-1}$.

HRMS (EI): calculated for $\mathrm{C}_{19} \mathrm{H}_{19} \mathrm{NO}\left(\mathrm{M}^{+}\right)$277.1467; found for $\mathrm{C}_{19} \mathrm{H}_{19} \mathrm{NO}\left(\mathrm{M}^{+}\right)$ 277.1452.

$\mathbf{R}_{\mathbf{f}}$ (Ether/Pet. Ether (5:95)): 0.27.

1-Acetyl-3-n-hexyl-2-(2-thiophene)indole (3o):<smiles>CC(=O)n1c(-c2cccs2)c(C#N)c2ccccc21</smiles>

The above procedure was followed and column chromatography was performed on silica gel with ether/pet. ether (5:95) as the solvent to afford the product in $71 \%$ yield.

${ }^{1}$ H NMR (400 MHz, $\mathbf{C D C l}_{3}, 293$ K): $\delta 8.44$ (dt, $\left.J=8.4 \mathrm{~Hz}, 1 \mathrm{H}\right), 7.56$ (br. d, $J=8.0 \mathrm{~Hz}$, 1H), 7.51 (dd, $J=5.2 \mathrm{~Hz}, 1.2 \mathrm{~Hz}, 1 \mathrm{H}$ ), 7.38 (ddd, $J=8.5 \mathrm{~Hz}, 7.2 \mathrm{~Hz}, 1.4 \mathrm{~Hz}, 1 \mathrm{H}$ ), 7.30 (ddd, $J=7.7 \mathrm{~Hz}, 7.6 \mathrm{~Hz}, 1.1 \mathrm{~Hz}, 1 \mathrm{H}$ ), 7.15 (dd, $J=5.2 \mathrm{~Hz}, 3.5 \mathrm{~Hz}, 1 \mathrm{H}$ ), 7.11 (dd, $J=3.5$ $\mathrm{Hz}, 1.2 \mathrm{~Hz}, 1 \mathrm{H}), 2.48$ (t, $J=8.0 \mathrm{~Hz}, 2 \mathrm{H}), 2.10$ (s, 3H), $1.63-1.56$ (m, 3H), $1.32-1.19$ (m, $5 \mathrm{H}), 0.85(\mathrm{t}, J=6.9 \mathrm{~Hz}, 3 \mathrm{H})$.

${ }^{13}$ C NMR (100 MHz, CDCl $_{3}, 293$ K): $\delta 171.0$ (C), 137.2 (C), $133.7(\mathrm{C}), 130.1$ (CH), $129.2(\mathrm{C}), 128.2(\mathrm{CH}), 127.4(\mathrm{CH}), 126.8(\mathrm{C}), 126.0(\mathrm{C}), 125.7(\mathrm{CH}), 123.4(\mathrm{CH}), 119.0$ (CH), $116.6(\mathrm{CH}), 31.6\left(\mathrm{CH}_{2}\right), 30.3\left(\mathrm{CH}_{2}\right), 29.4\left(\mathrm{CH}_{2}\right), 26.4\left(\mathrm{CH}_{3}\right), 24.6\left(\mathrm{CH}_{2}\right), 22.6$ $\left(\mathrm{CH}_{2}\right), 14.1\left(\mathrm{CH}_{3}\right)$.

FTIR: 2954, 1698, 1454, 1305, $750 \mathrm{~cm}^{-1}$.

HRMS (EI): calculated for $\mathrm{C}_{20} \mathrm{H}_{23} \mathrm{NOS}\left(\mathrm{M}^{+}\right)$325.1500; found for $\mathrm{C}_{20} \mathrm{H}_{23} \mathrm{NOS}\left(\mathrm{M}^{+}\right)$ 325.1512.

$\mathbf{R}_{\mathbf{f}}$ (Ether/Pet. Ether (5:95)): 0.29. 


\section{1-Acetyl-3-n-hexyl-2-(5- $N$-tosylindolyl)indole (3p):}

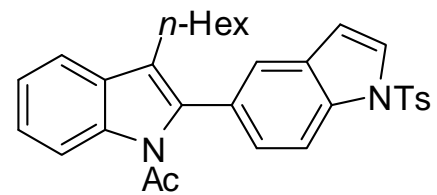

The above procedure was followed and column chromatography was performed on silica gel with ether/pet. ether (20:80) as the solvent to afford the product in $81 \%$ yield.

${ }^{1}$ H NMR (400 MHz, CDCl $_{3}, 293$ K): $\delta 8.42(\mathrm{~d}, J=8.0 \mathrm{~Hz}, 1 \mathrm{H}), 8.01(\mathrm{~d}, J=8.1 \mathrm{~Hz}$, 1H), 7.83 (d, $J=8.4 \mathrm{~Hz}, 2 \mathrm{H}), 7.65$ (d, $J=3.7 \mathrm{~Hz}, 1 \mathrm{H}$ ), 7.56 (br. s, $1 \mathrm{H}$ ), 7.54 (br. s, 1H), $7.38-7.27$ (m, 5H), 6.70 (dd, $J=3.7 \mathrm{~Hz}, 0.7 \mathrm{~Hz}, 1 \mathrm{H}), 2.49$ (t, $J=7.6 \mathrm{~Hz}, 2 \mathrm{H}), 2.38$ (s, $3 \mathrm{H}), 1.81$ (s, 3H), $1.58-1.51(\mathrm{~m}, 3 \mathrm{H}), 1.26-1.11(\mathrm{~m}, 5 \mathrm{H}), 0.77$ (t, $J=7.0 \mathrm{~Hz}, 3 \mathrm{H})$.

${ }^{13}$ C NMR (100 MHz, $\mathbf{C D C l}_{3}, 293$ K): $\delta 171.1$ (C), 145.3 (C), 136.9 (C), 135.3 (C), 134.7 (C), 134.6 (C), 130.9 (C), 130.0 (CH), 129.6 (C), 128.7 (C), 127.5 (CH), 126.9 $(\mathrm{CH}), 126.8(\mathrm{CH}), 125.2(\mathrm{CH}), 123.4(\mathrm{CH}), 123.2(\mathrm{C}), 123.1(\mathrm{CH}), 118.9(\mathrm{CH}), 116.5$ $(\mathrm{CH}), 113.7(\mathrm{CH}), 108.9(\mathrm{CH}), 31.4\left(\mathrm{CH}_{2}\right), 30.1\left(\mathrm{CH}_{2}\right), 29.2\left(\mathrm{CH}_{2}\right), 27.7\left(\mathrm{CH}_{3}\right), 24.2$ $\left(\mathrm{CH}_{2}\right), 22.5\left(\mathrm{CH}_{2}\right), 21.6\left(\mathrm{CH}_{3}\right), 14.0\left(\mathrm{CH}_{3}\right)$.

FTIR: 2927, 2856, 1696, 1448, 1372, $1307 \mathrm{~cm}^{-1}$.

HRMS (EI): calculated for $\mathrm{C}_{31} \mathrm{H}_{32} \mathrm{~N}_{2} \mathrm{O}_{3} \mathrm{~S}\left(\mathrm{M}^{+}\right)$512.2134; found for $\mathrm{C}_{31} \mathrm{H}_{32} \mathrm{~N}_{2} \mathrm{O}_{3} \mathrm{~S}\left(\mathrm{M}^{+}\right)$ 512.2128 .

$\mathbf{R}_{\mathbf{f}}$ (Ether/Hexanes (20:80)): 0.23.

\section{Representative examples for the Deacetylation of $N$-Acetylindoles:}

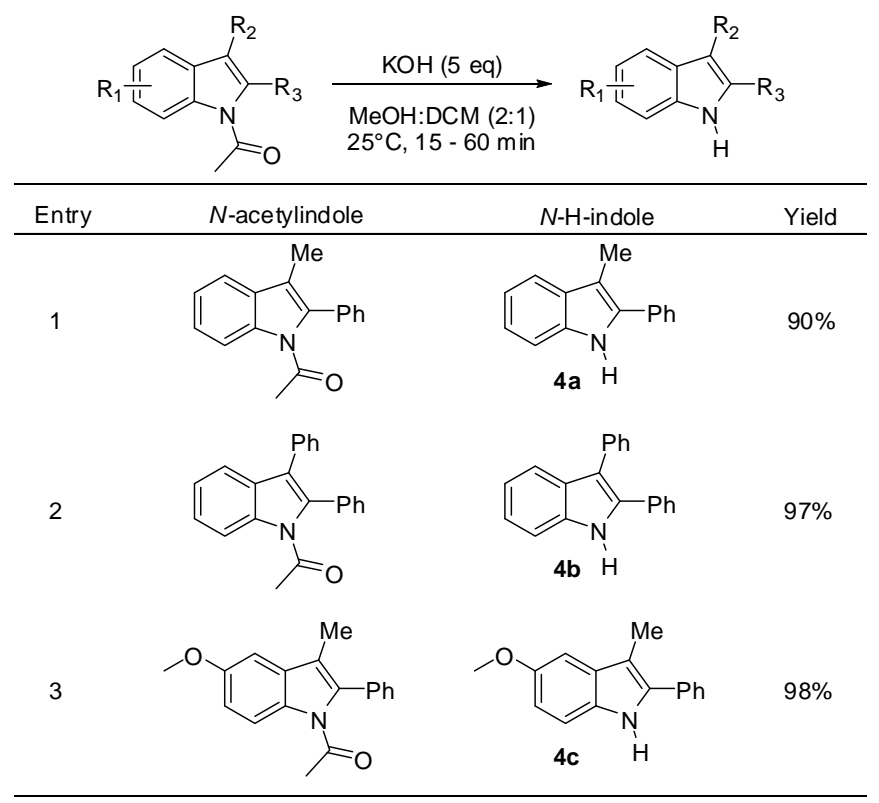

\section{Representative Procedure for the deacetylation of $N$-Acetylindoles:}

To a 4 dram vial containing a magnetic stir bar was weighed out 1-Acetyl-3methyl-2-phenylindole (3a) (0.1004 g, $0.4 \mathrm{mmol}, 1 \mathrm{eq})$. Methanol (2 mL) and 
dichloromethane $(1 \mathrm{~mL})$ (total concentration: $0.13 \mathrm{M}$ ) were added and 3a dissolved. Potassium hydroxide ( $0.1278 \mathrm{~g}, 2 \mathrm{mmol}, 5 \mathrm{eq})$ was added to the stirring reaction mixture and it was monitored by TLC analysis until deemed complete. After 1 hour (or specified time) of stirring at room temperature the reaction was diluted with dichloromethane and washed with a saturated solution of ammonium chloride. The organics were dried over $\mathrm{MgSO}_{4}$ and the solvent removed under reduced pressure. The residue was loaded onto silica and flashed over silica with Ether/Pet. Ether.

\section{3-Methyl-2-phenylindole (4a):}<smiles>Cc1c(-c2ccccc2)[nH]c2ccccc12</smiles>

The above procedure was followed to afford the product in $90 \%$ yield. This compound can be purchased from commercial sources (CAS: 10257-92-8) and spectral data was consistent with that previously reported. ${ }^{6}$

${ }^{1}$ H NMR (400 MHz, CDCl $_{3}, 293$ K): $\delta 7.97$ (br. s, 1H), 6.61 - 6.54 (m, 3H), 7.48 - 7.44 (m, 2H), $7.36-7.32$ (m, 2H), 7.20 (ddd, $J=J=7.1 \mathrm{~Hz}, J=1.6 \mathrm{~Hz}, 1 \mathrm{H}), 7.14$ (ddd, $J=J$ $=7.4 \mathrm{~Hz}, J=1.2 \mathrm{~Hz}, 1 \mathrm{H}), 2.45(\mathrm{~s}, 3 \mathrm{H})$.

\section{2,3-Diphenylindole (4b):}<smiles>c1ccc(-c2[nH]c3ccccc3c2-c2ccccc2)cc1</smiles>

The above procedure was followed to afford the product in $97 \%$ yield. This compound can be purchased from commercial sources (CAS: 3469-20-3) and spectral data was consistent with that previously reported. ${ }^{7}$

${ }^{1}$ H NMR (400 MHz, CDCl 3 , 293 K): $\delta 8.14$ (br. s, 1H), 7.68 (d, $J=7.6$ Hz, 1H), 7.44 7.33 (m, 7H), 7.31 - 7.19 (m, 5H), 7.15 (t, $J=8.0 \mathrm{~Hz}, 1 \mathrm{H})$.

\section{5-Methoxy-3-methyl-2-phenylindole (4c):}<smiles>COc1ccc2[nH]c(-c3ccccc3)c(C)c2c1</smiles>

The above procedure was followed with stirring for 15 minutes to afford the product in $98 \%$ yield. Spectral data was consistent with that previously reported. ${ }^{8}$ 
${ }^{1}$ H NMR (400 MHz, CDCl $_{3}, 293$ K): $\delta 7.90$ (br. s, 1H), $7.56-7.53$ (m, 2H), $7.48-7.42$ (m, 2H), 7.33 (tt, $J=7.4 \mathrm{~Hz}, J=1.3 \mathrm{~Hz}, 1 \mathrm{H}), 7.23(\mathrm{~d}, J=8.7 \mathrm{~Hz}, 1 \mathrm{H}), 7.03(\mathrm{~d}, J=2.5$ Hz, 1H), 6.86 (dd, $J=8.7 \mathrm{~Hz}, J=2.5 \mathrm{~Hz}, 1 \mathrm{H}$ ), 3.88 (s, 3H), 2.42 (s, 3H).

\section{Mechanistic Studies:}

\section{Synthesis of acetanilide- $d_{5}\left(1 \mathrm{a}-d_{5}\right)$ :}

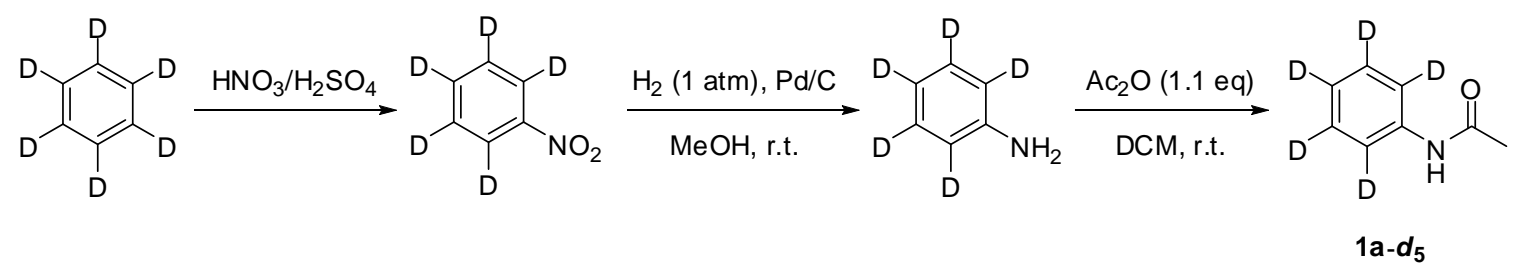

Concentrated nitric acid and concentrated sulfuric acid $(1: 1 \mathrm{v} / \mathrm{v})$ were mixed together in a round-bottom flask held in an ice-water bath. Benzene- $d_{6}(2.0 \mathrm{~mL}, 23 \mathrm{mmol}, 1 \mathrm{eq})$ was added drop-wise at room temperature and the reaction was allowed to stir for an additional 15 minutes. The reaction mixture was poured into ice-water and extracted with DCM. Nitration was determined by GCMS and the product was used without further purification.

Crude nitrobenzene- $d_{5}$ was dissolved in $\mathrm{MeOH}$ and purged with argon. $\mathrm{Pd} / \mathrm{C}$ (10 mol\% $\mathrm{Pd}$ ) was added and the reaction was purged with $\mathrm{H}_{2}$ (1 atm) for 10 minutes and then held under an $\mathrm{H}_{2}$ atmosphere overnight at room temperature. The reaction mixture was checked by TLC and filtered over celite and the solvent removed under reduced pressure. The crude aniline- $d_{5}$ was used without further purification.

Crude aniline- $d_{5}$ was added to a round-bottom flask via syringe and fitted with a rubber septa. The flask was purged with argon and dry DCM was added. Acetic anhydride was added and the reaction was stirred at room temperature and monitored by TLC. Upon completion the reaction mixture was washed with a saturated solution of sodium carbonate, the organic layers dried with $\mathrm{MgSO}_{4}$ and the solvent removed under reduced pressure. The product was obtained in $48 \%$ yield (over 3 steps, based on amount of benzene- $\left.d_{6}\right)(1.5045 \mathrm{~g})$. Purified by flash chromatography on silica gel with ethyl acetate : pet. ether $(40: 60)$. This compound can be purchased from commercial sources (CAS: 15826-91-2).

${ }^{1}$ H NMR (400 MHz, $\mathbf{C D C l}_{3}, 293$ K): $\delta 7.59$ (br. s, 1H), 2.16 (s, 3H).

\section{Deuterium loss experiments on 1a-d $d_{5}$ :}


<smiles>[2H]c1c([2H])c([2H])c(N([CH2-])[CH+]c2ccccc2)c([2H])c1[2H]</smiles>

The general procedure above was followed however the reaction was stopped after 2 minutes. Both product formed and remaining starting material were isolated by flash chromatography on silica gel (ether:pet. ether, 5:95 (product, 3a- $\boldsymbol{d}_{3}$ ); then ethyl acetate:pet. ether, 40:60 (starting material, $\left.1 \mathbf{a}-\boldsymbol{d}_{3}\right)$ ) and compared with authentic samples by ${ }^{1} \mathrm{H}$ NMR spectroscopy (300 MHz).
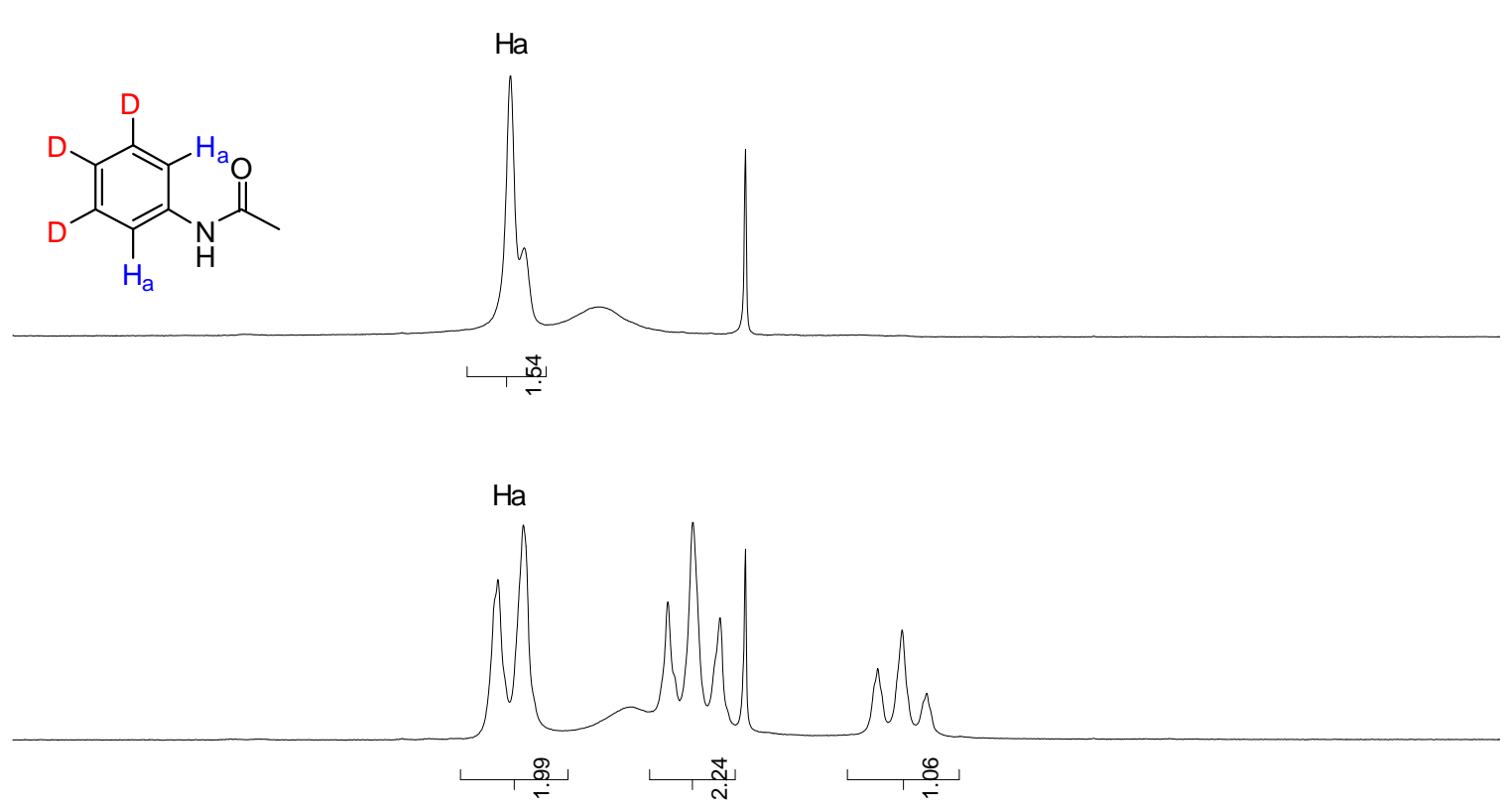

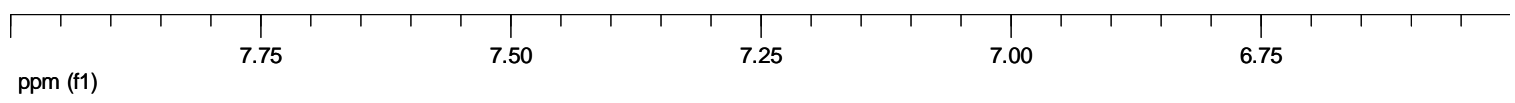



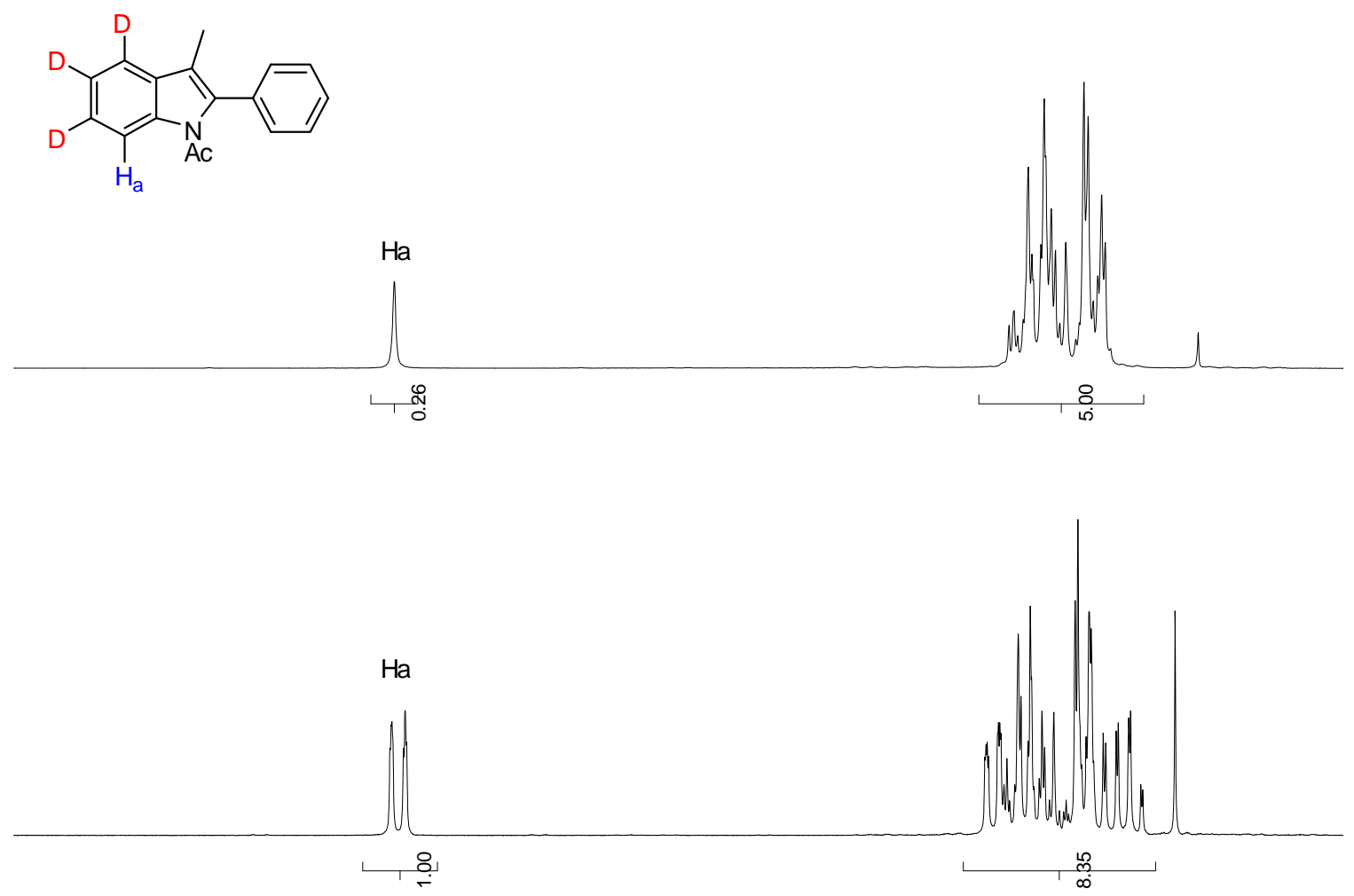

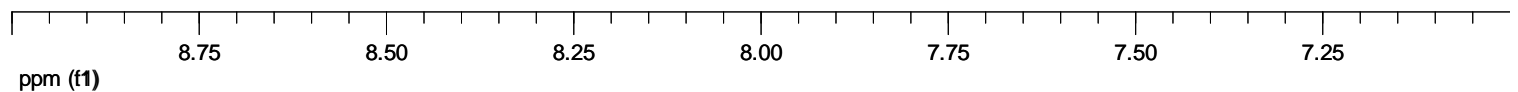

Deuterium incorporation on unsymmetrical acetanilides (1i):<smiles>CC#CC#Cc1ccccc1</smiles>

The general procedure above was followed however the reaction was stopped after 3 minutes. Both product formed and remaining starting material were isolated by flash chromatography on silica gel (ether:pet. ether, 10:90 (product, 3i-6-OMe-d); then ethyl acetate:pet. ether, 50:50 (starting material, 1i-d)) and compared with authentic samples by ${ }^{1} \mathrm{H}$ NMR spectroscopy (300 $\mathrm{MHz}$, in $\mathrm{CDCl}_{3}$ unless otherwise specified). 
$300 \mathrm{MHz}$ (acetone-d6)<smiles>COc1ccc(P)c(NC(C)=O)c1P</smiles>

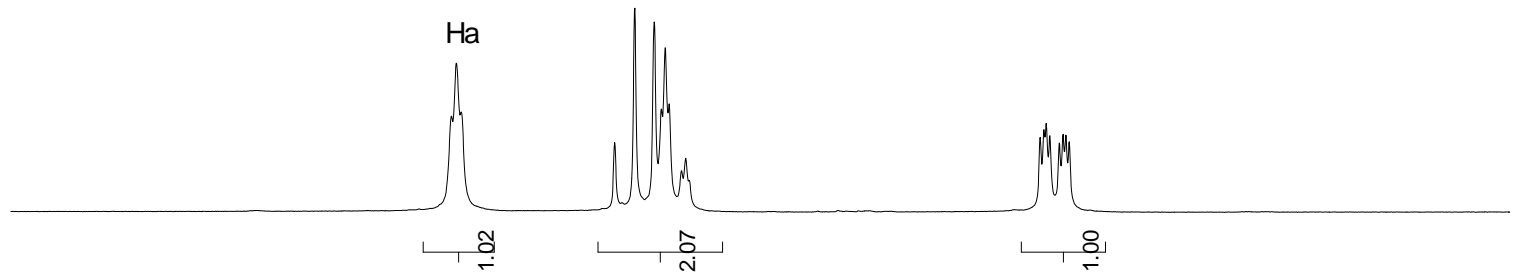

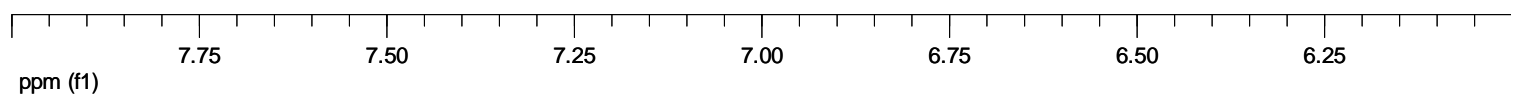<smiles>COc1ccc(C)c(NC(C)=O)c1C</smiles>
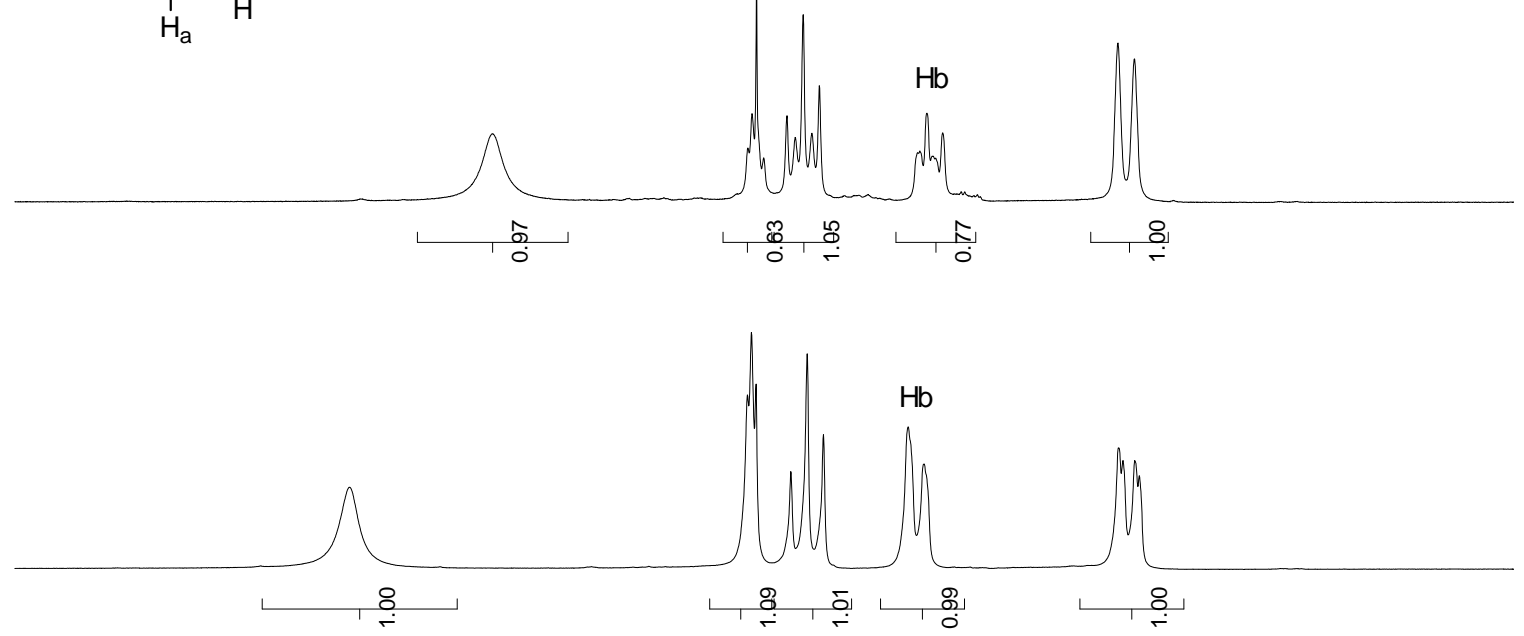

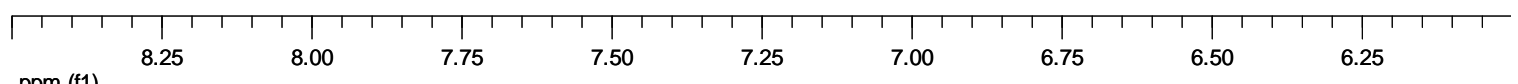
ppm (f1) 

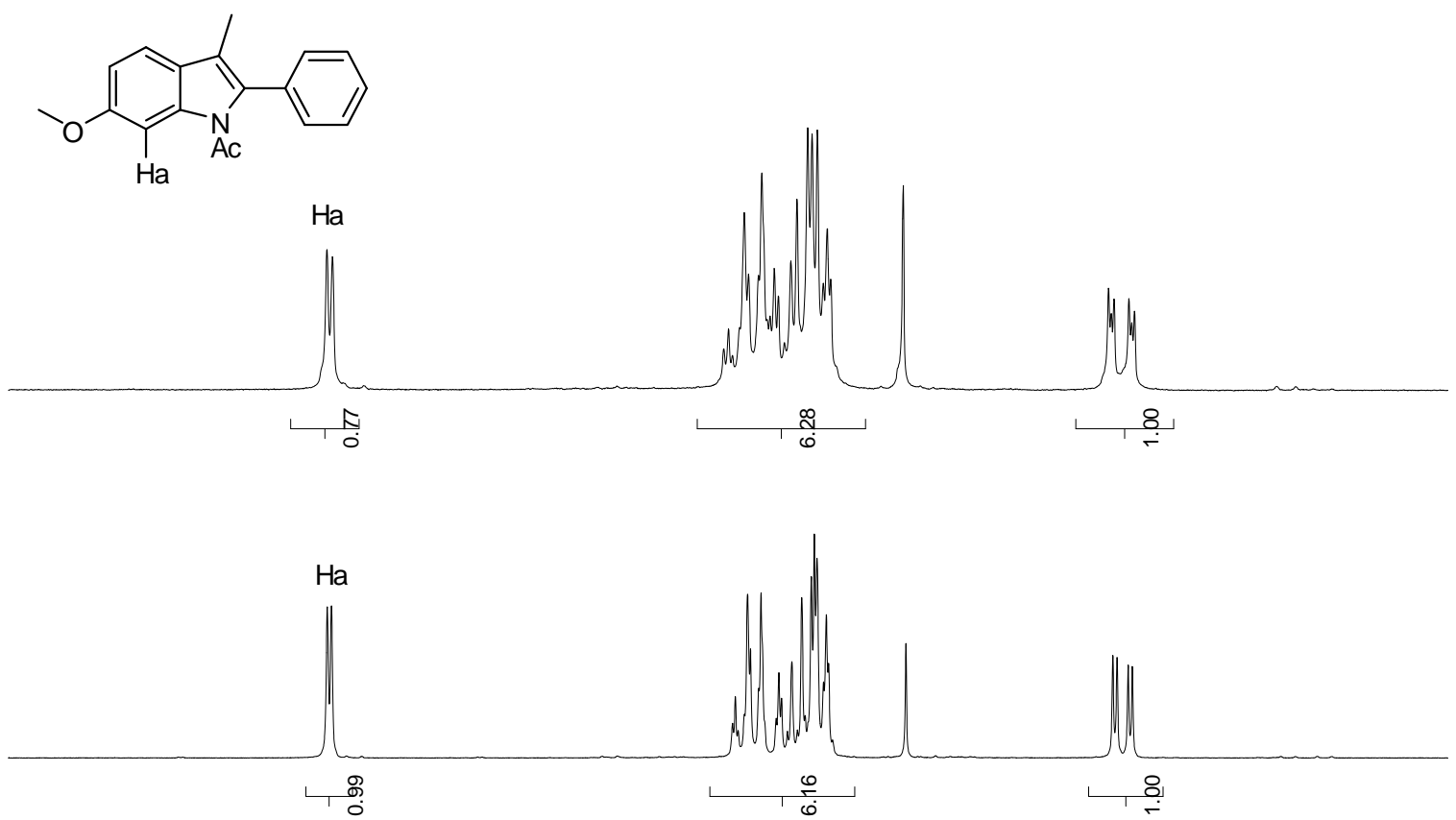

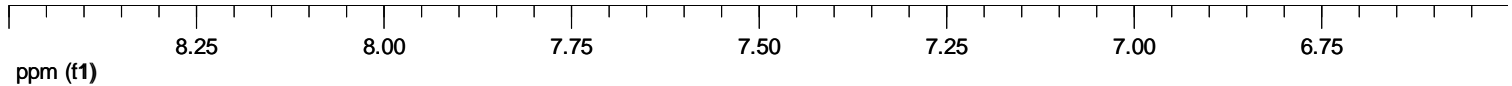



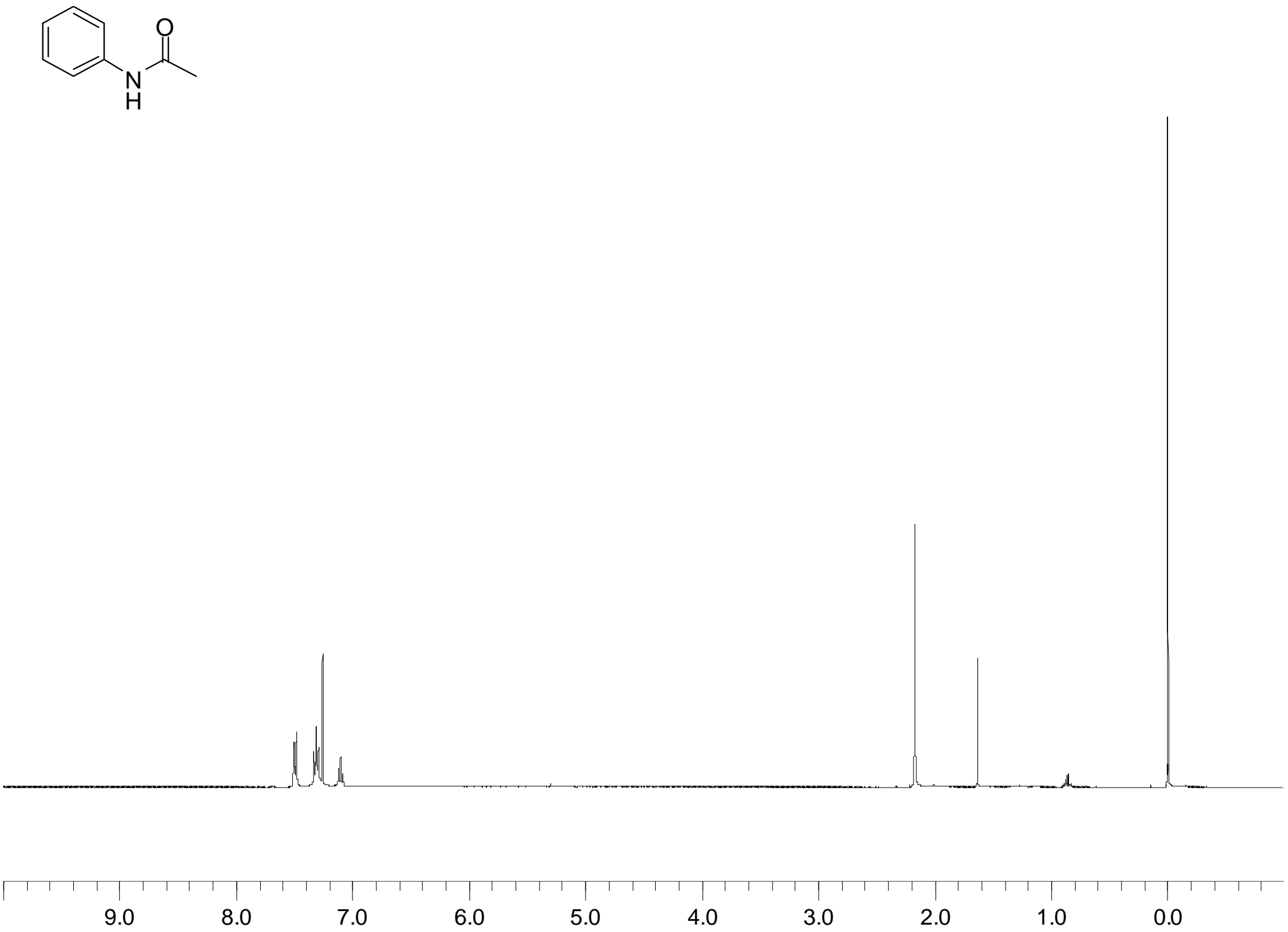

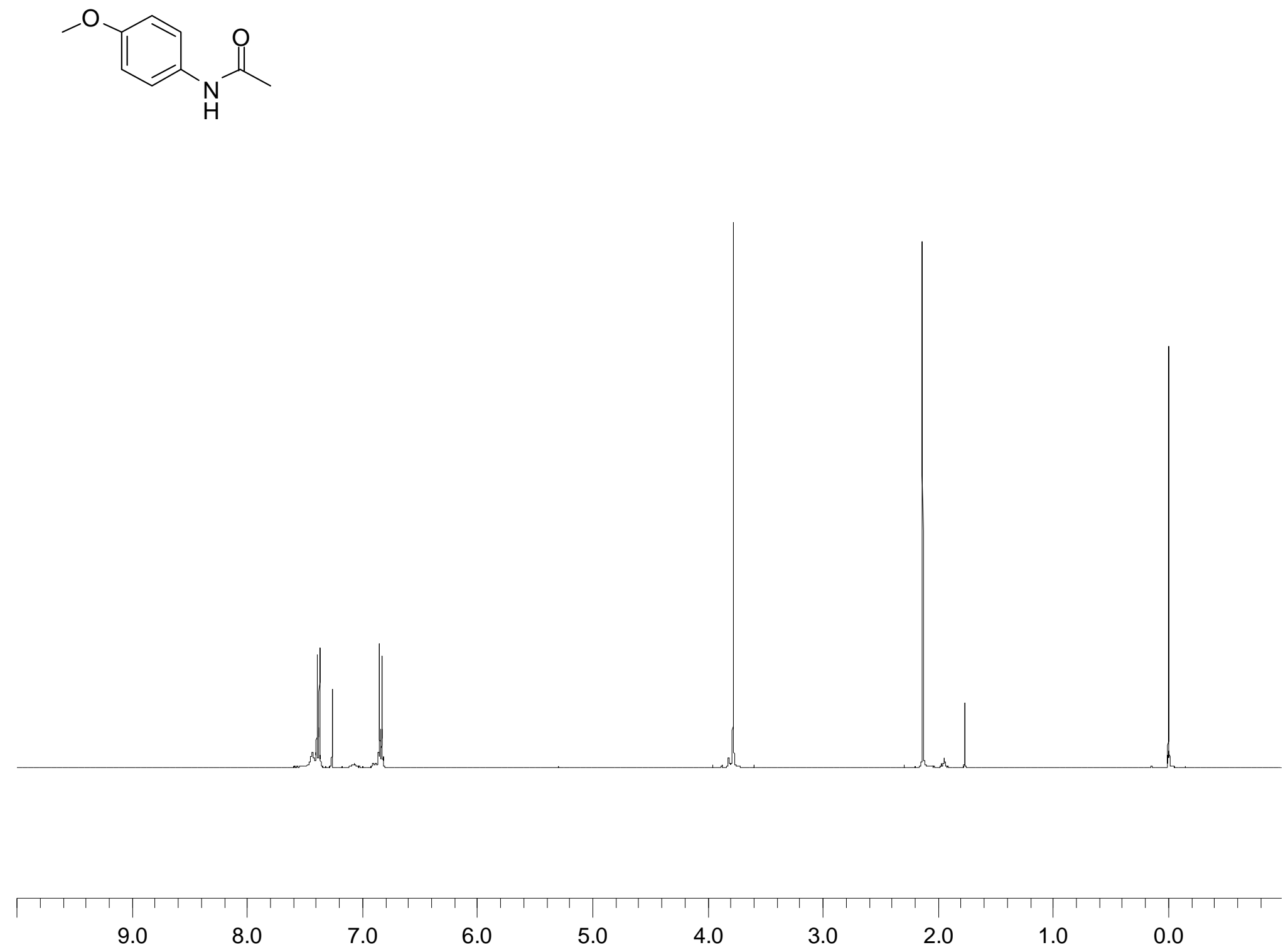


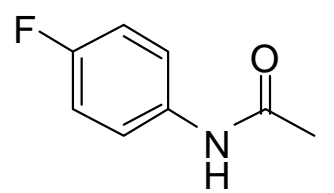

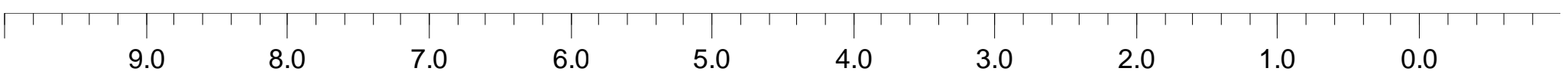


$\mathrm{MeO}_{2} \mathrm{C}$

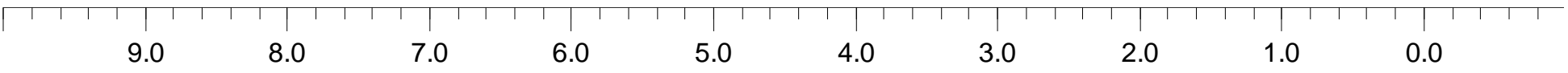


$\mathrm{H}_{\mathrm{H}}^{\mathrm{Cl}}$

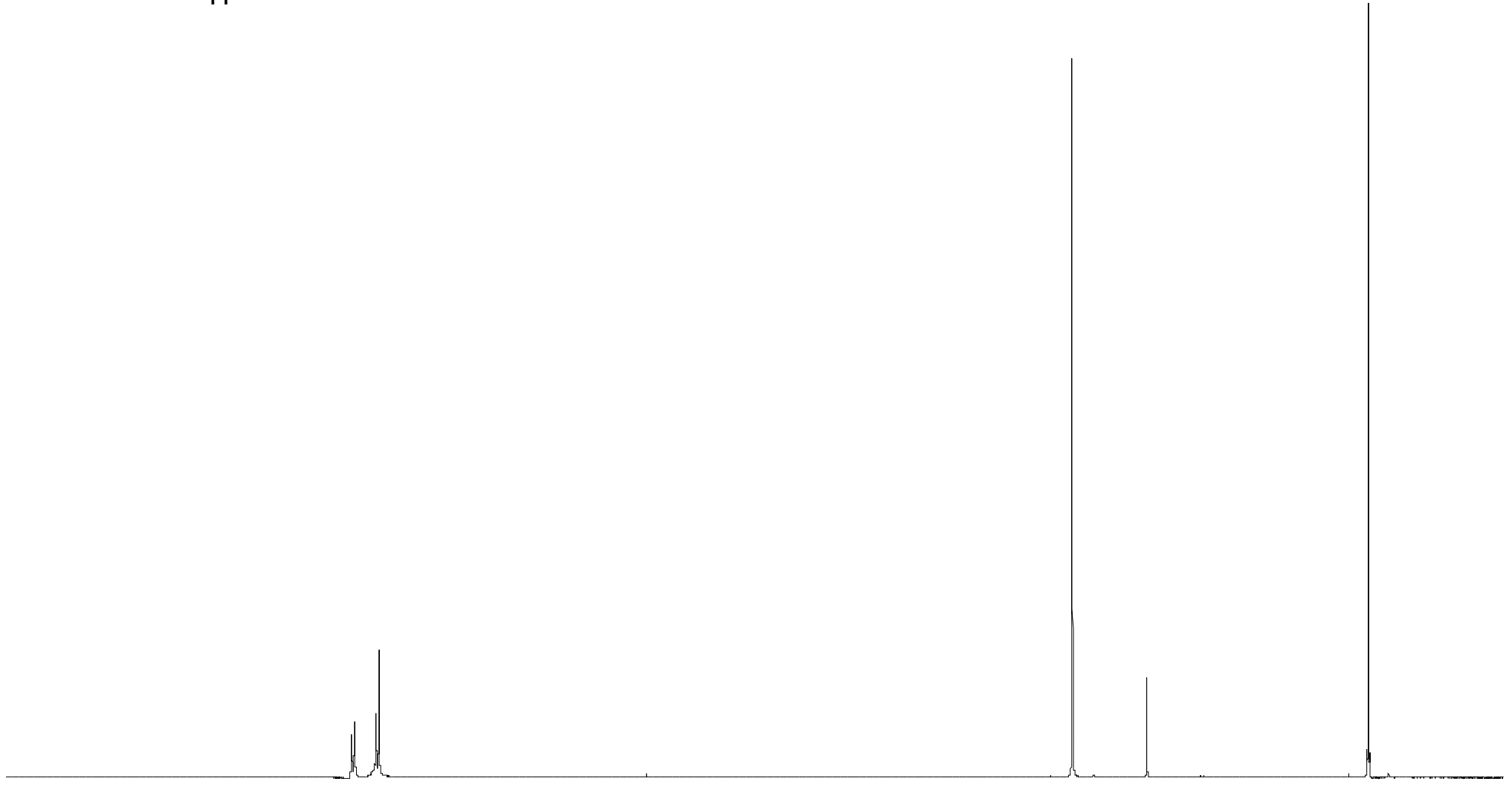

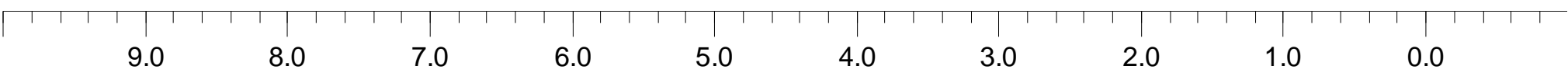




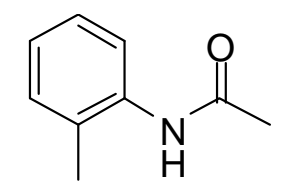

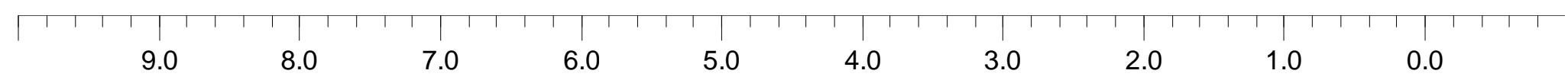


S29
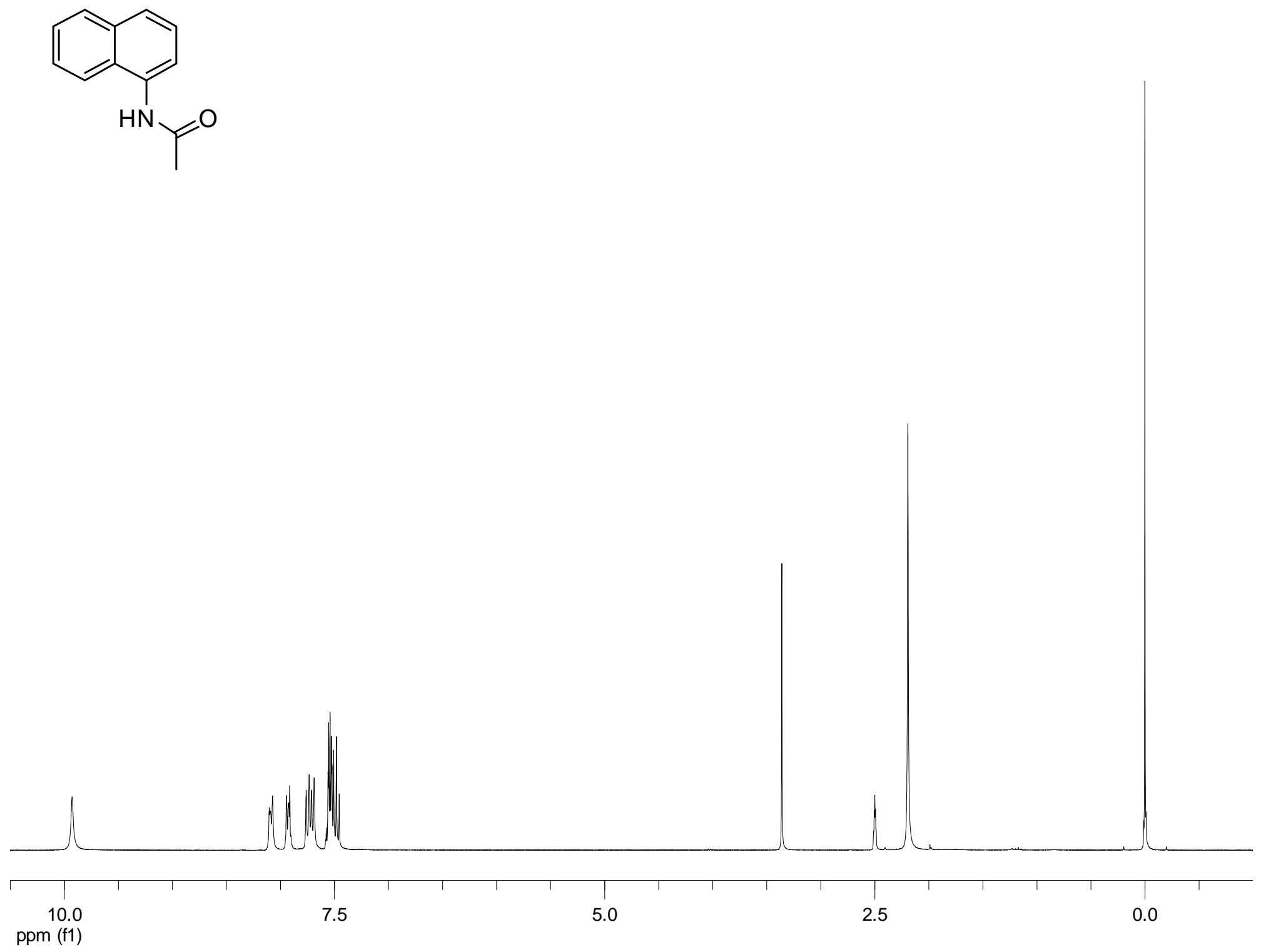


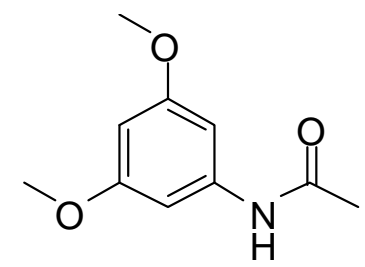

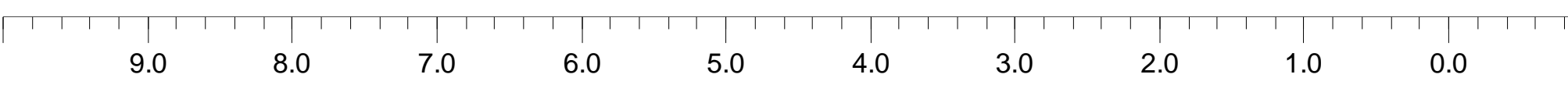




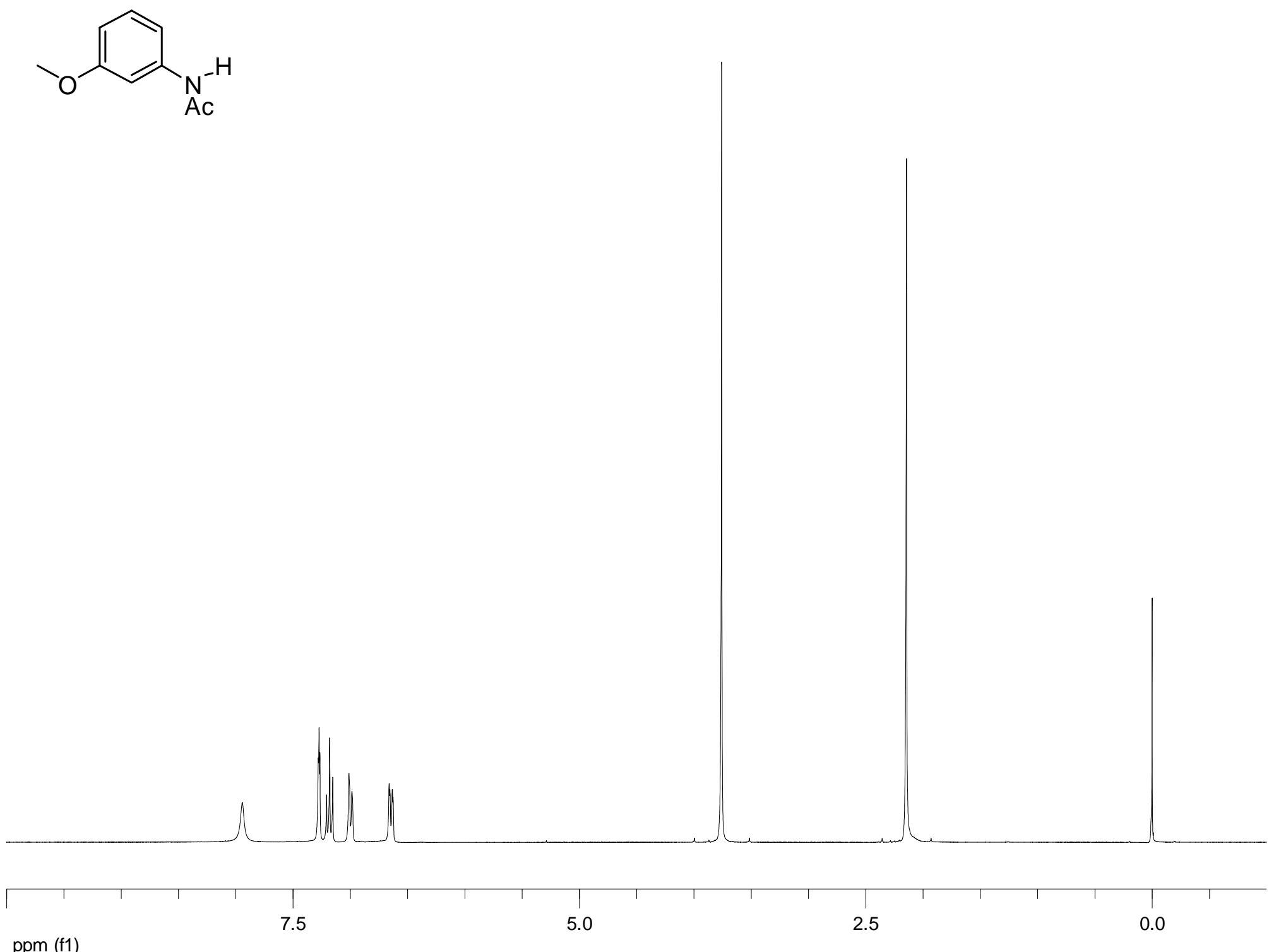

ppm (f1) 

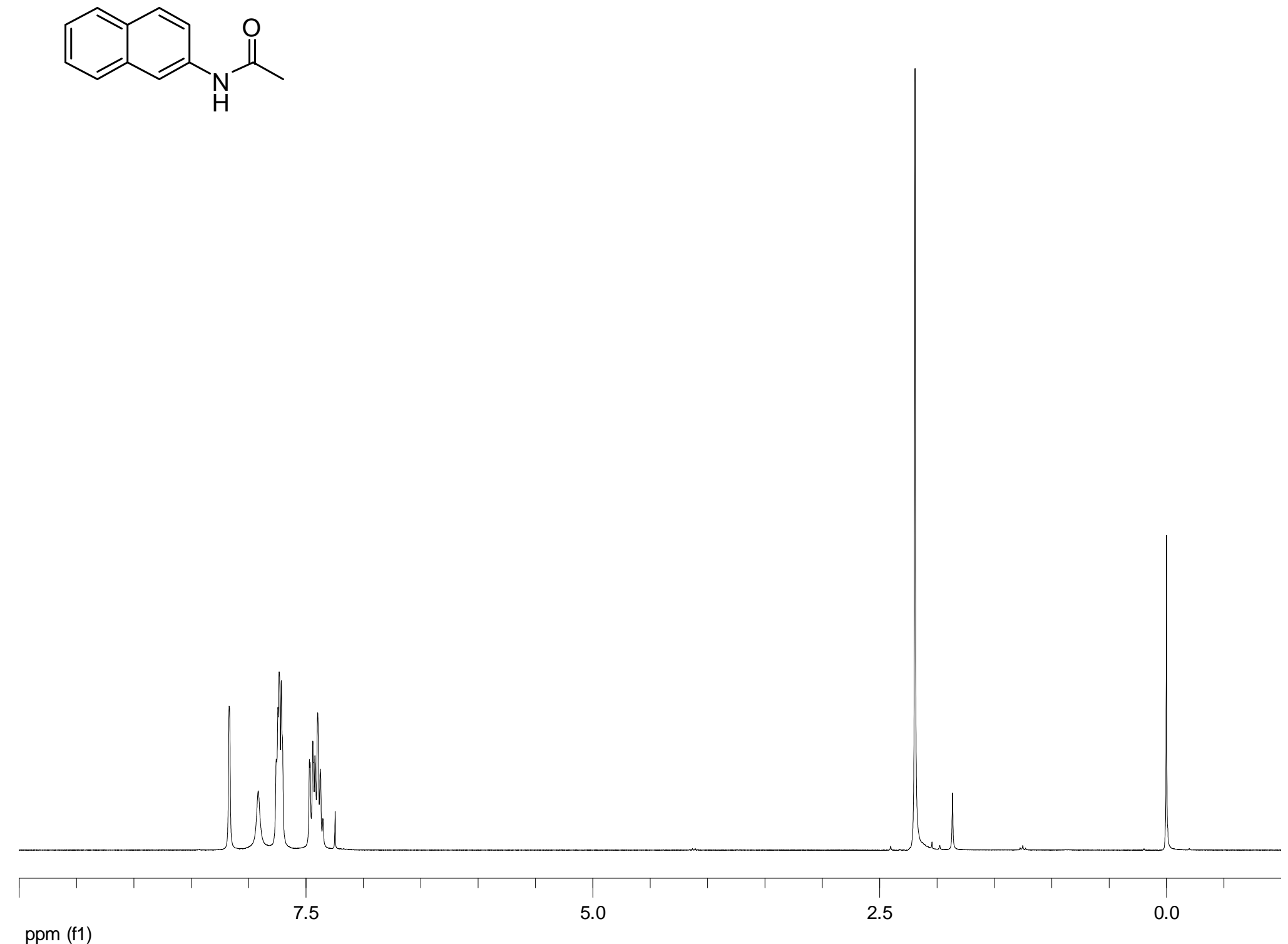

ppm (f1) 
S33
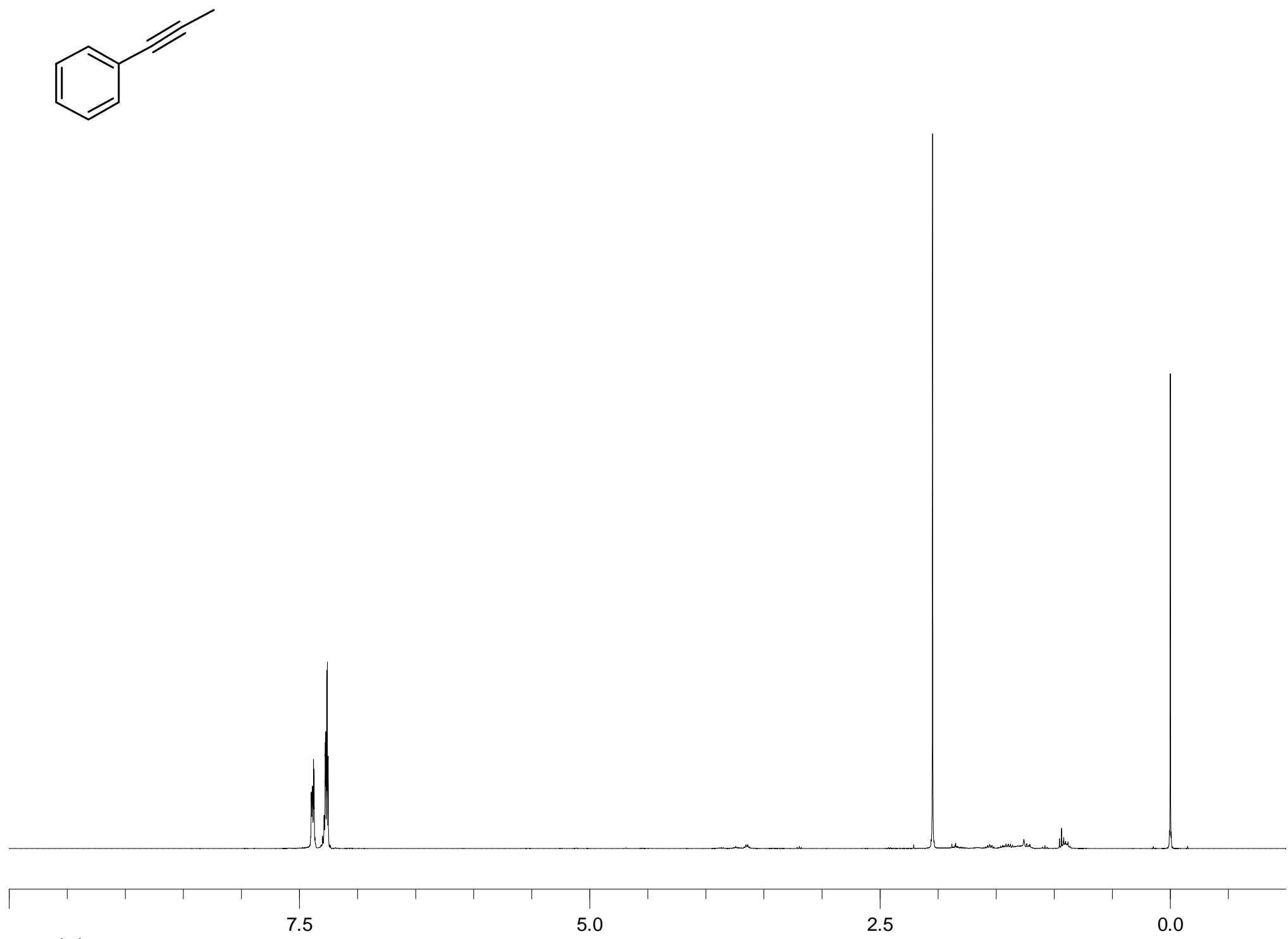

ppm (t1) 


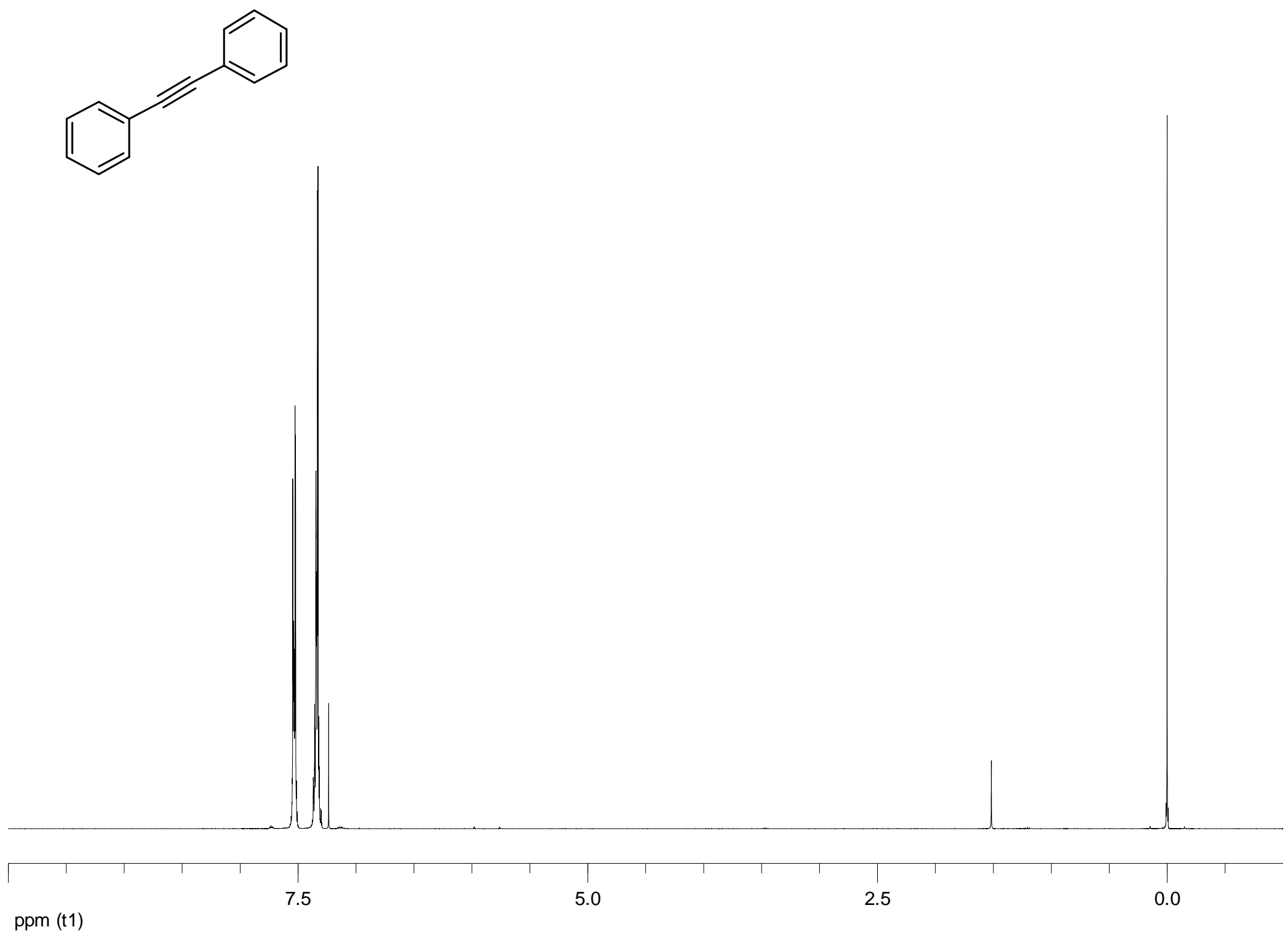


$\left\langle{ }_{s} \approx{ }_{n-h e x}\right.$

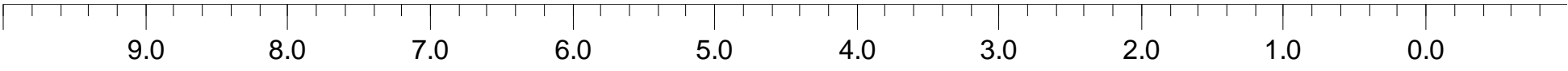



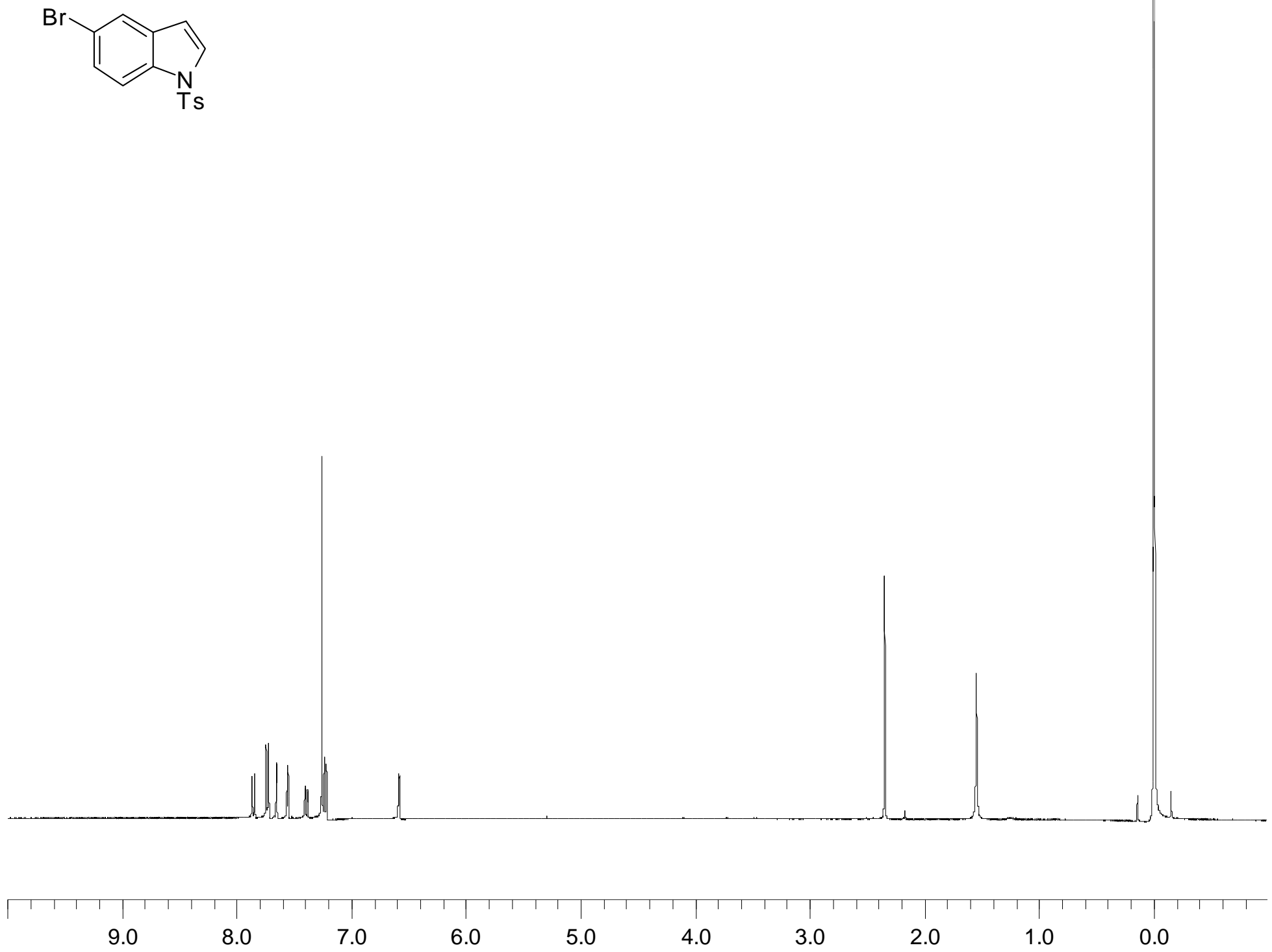
n-hex
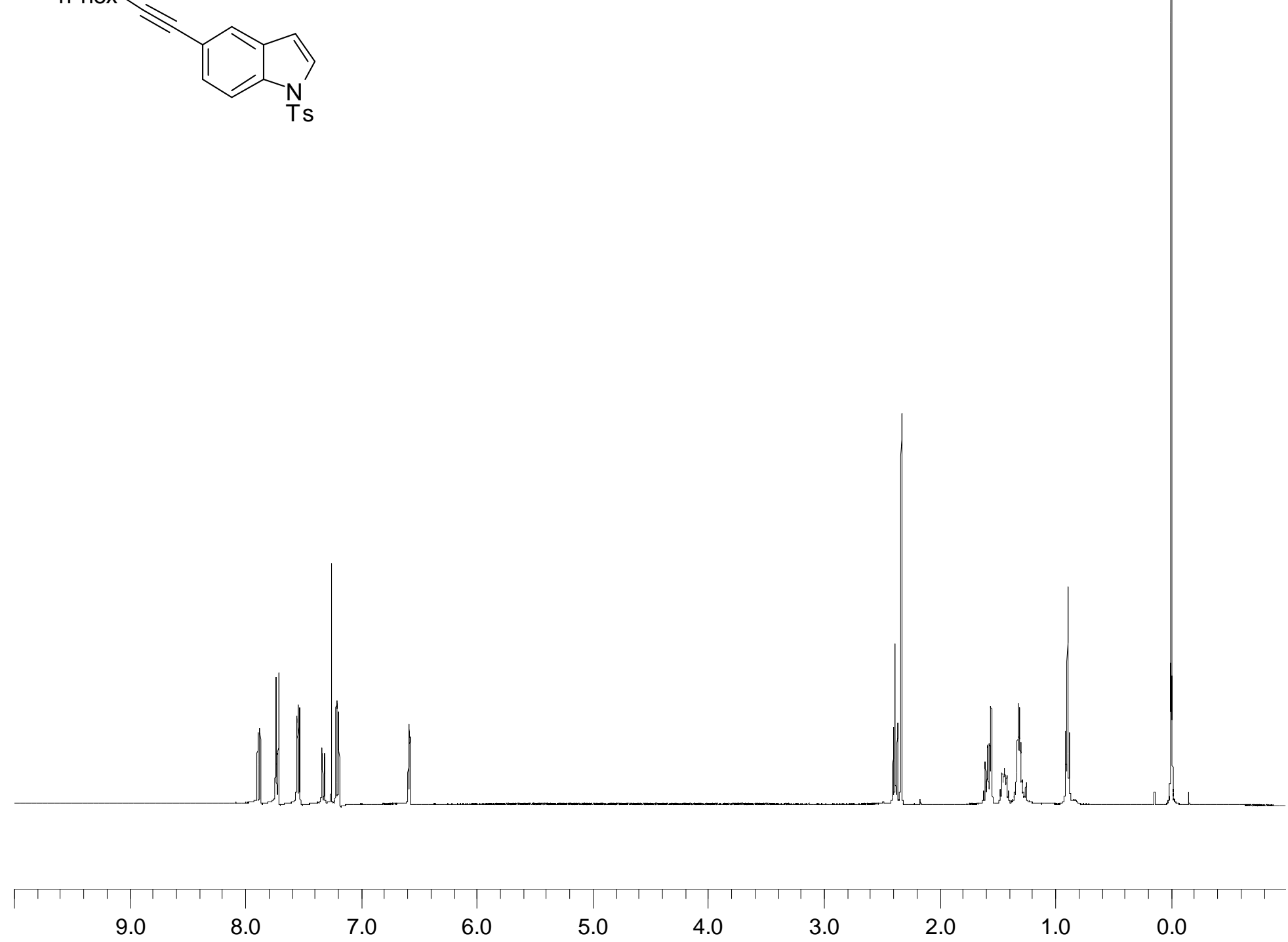

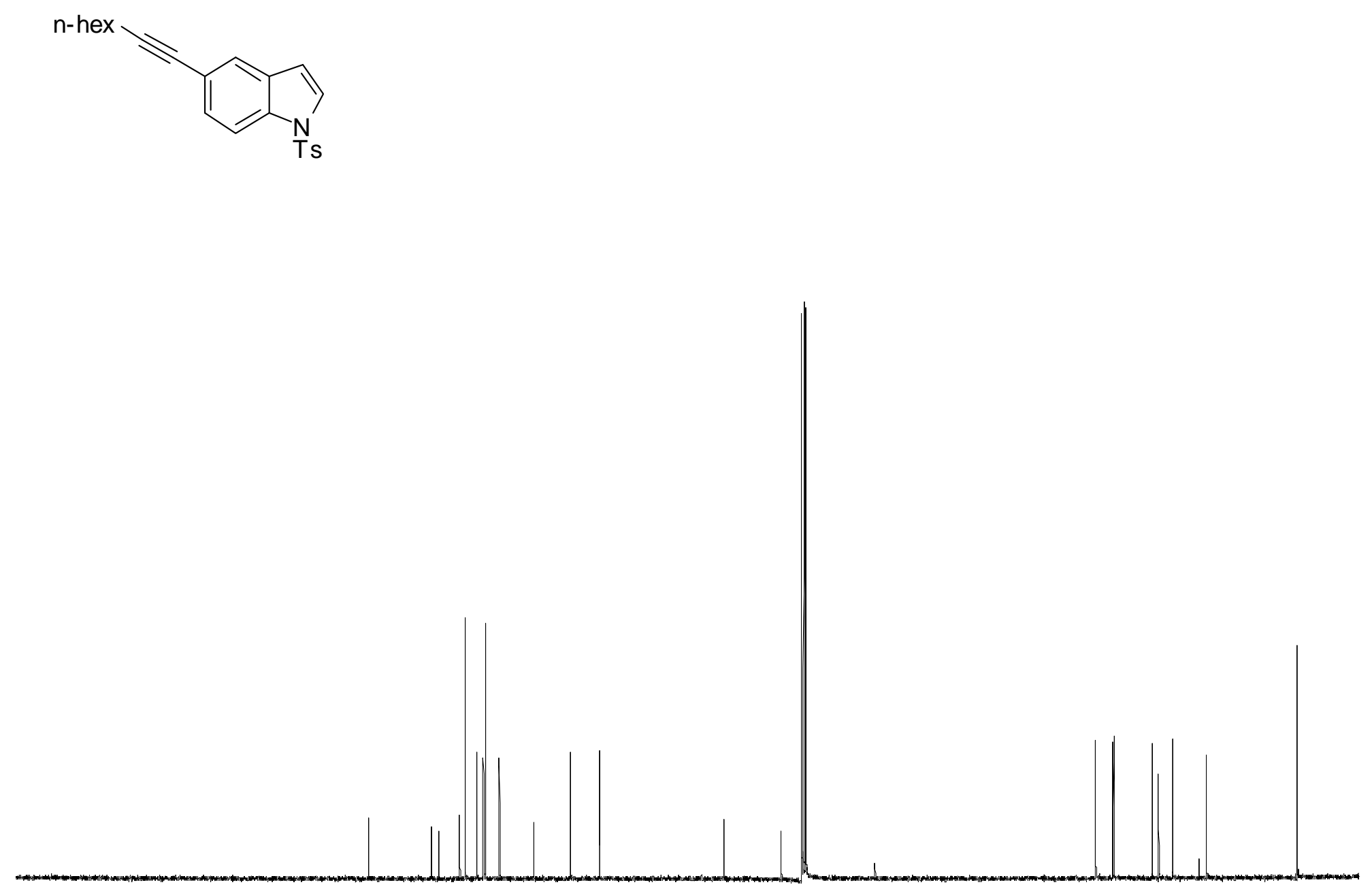

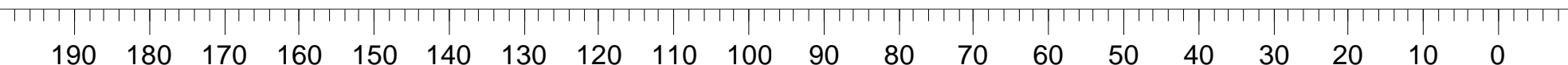



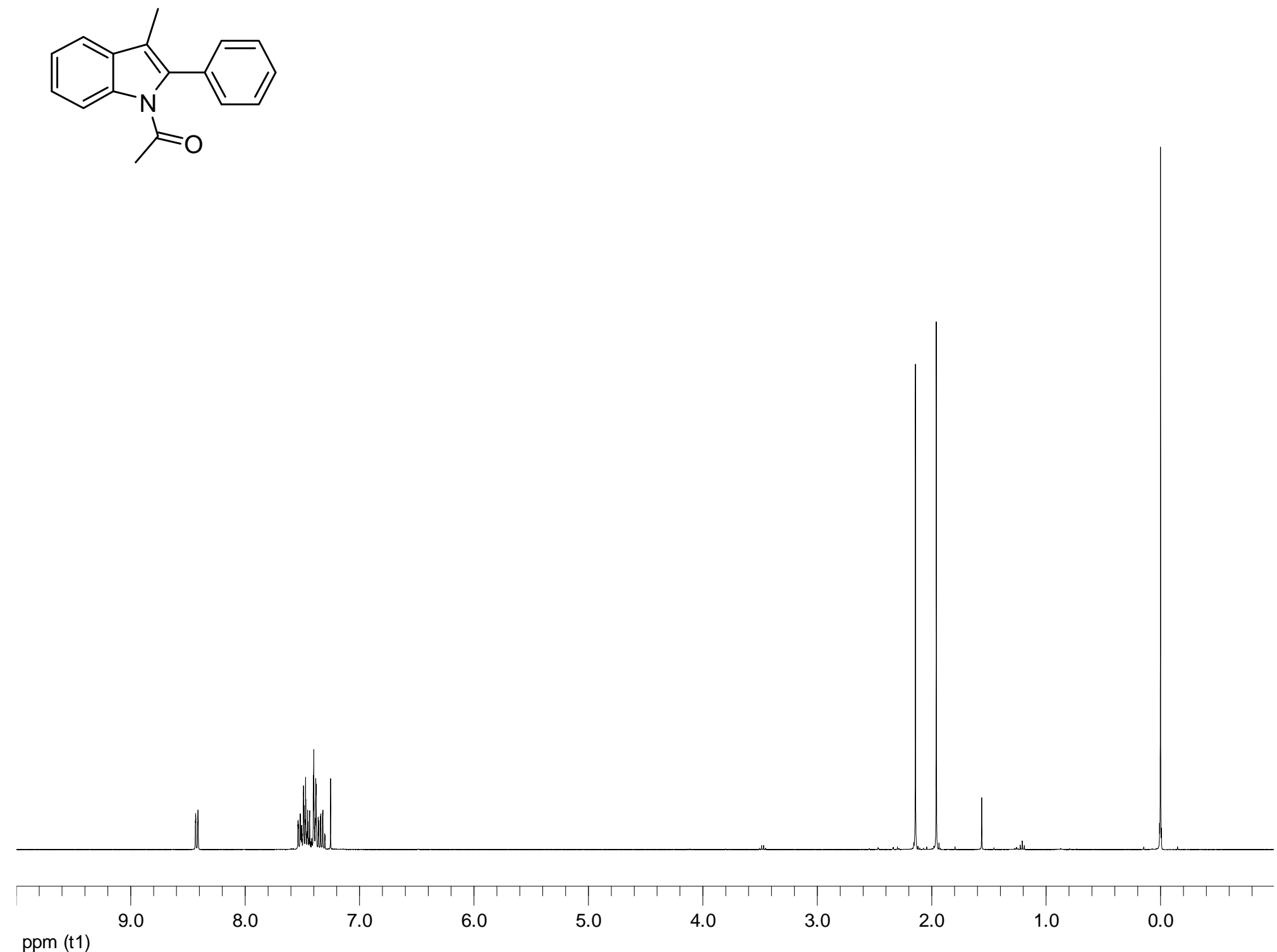

ppm (t1) 

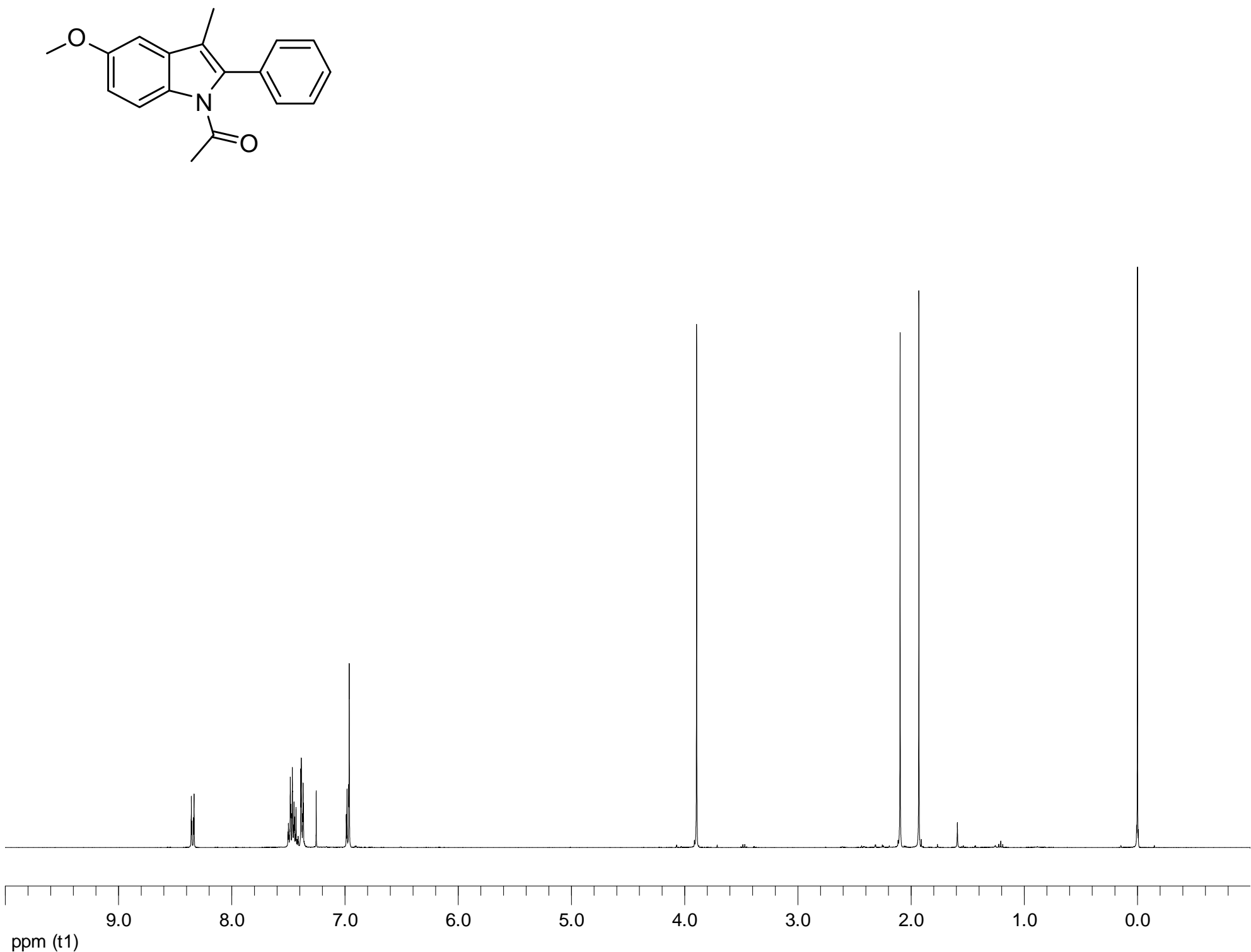

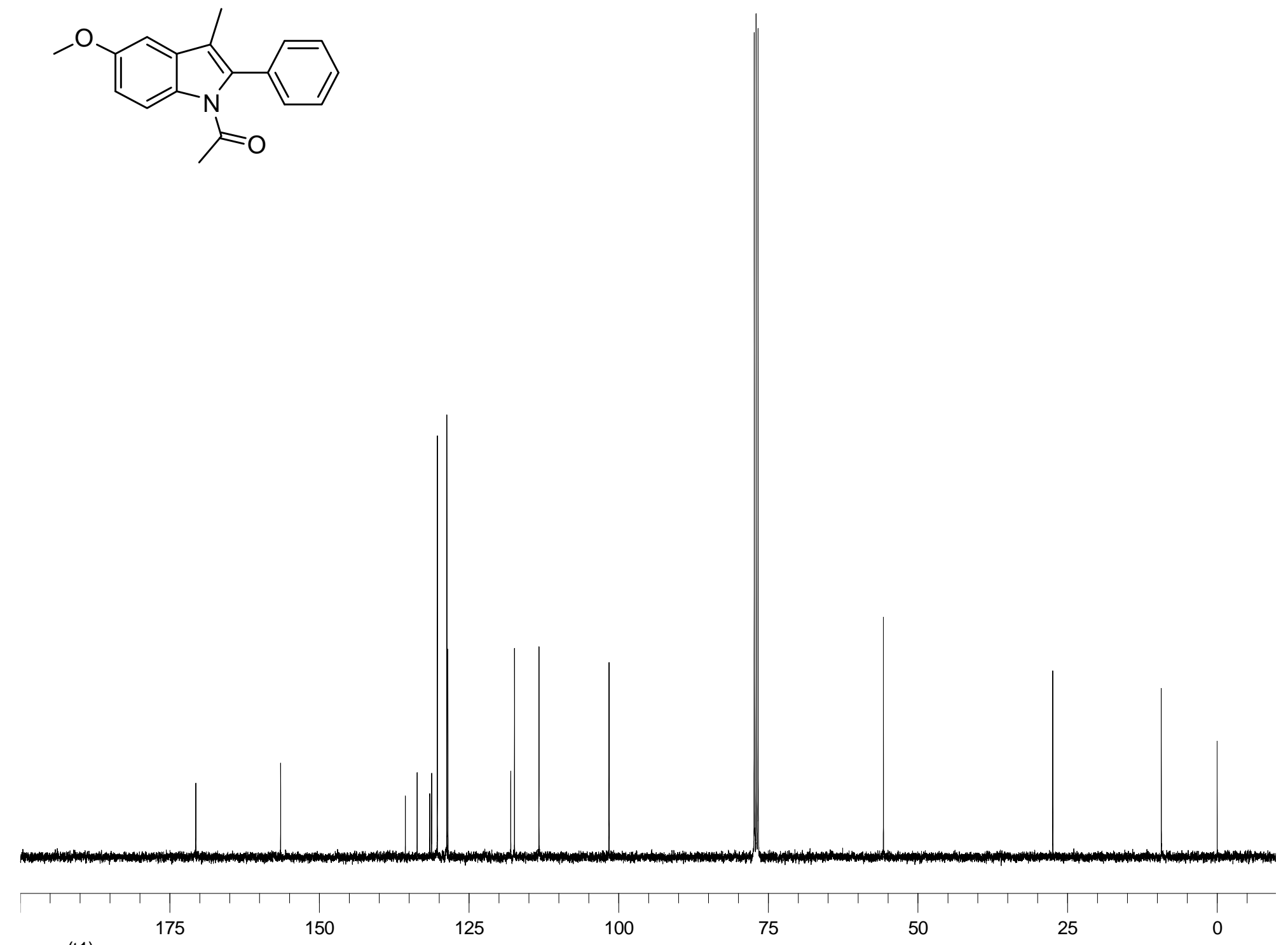

ppm (t1) 

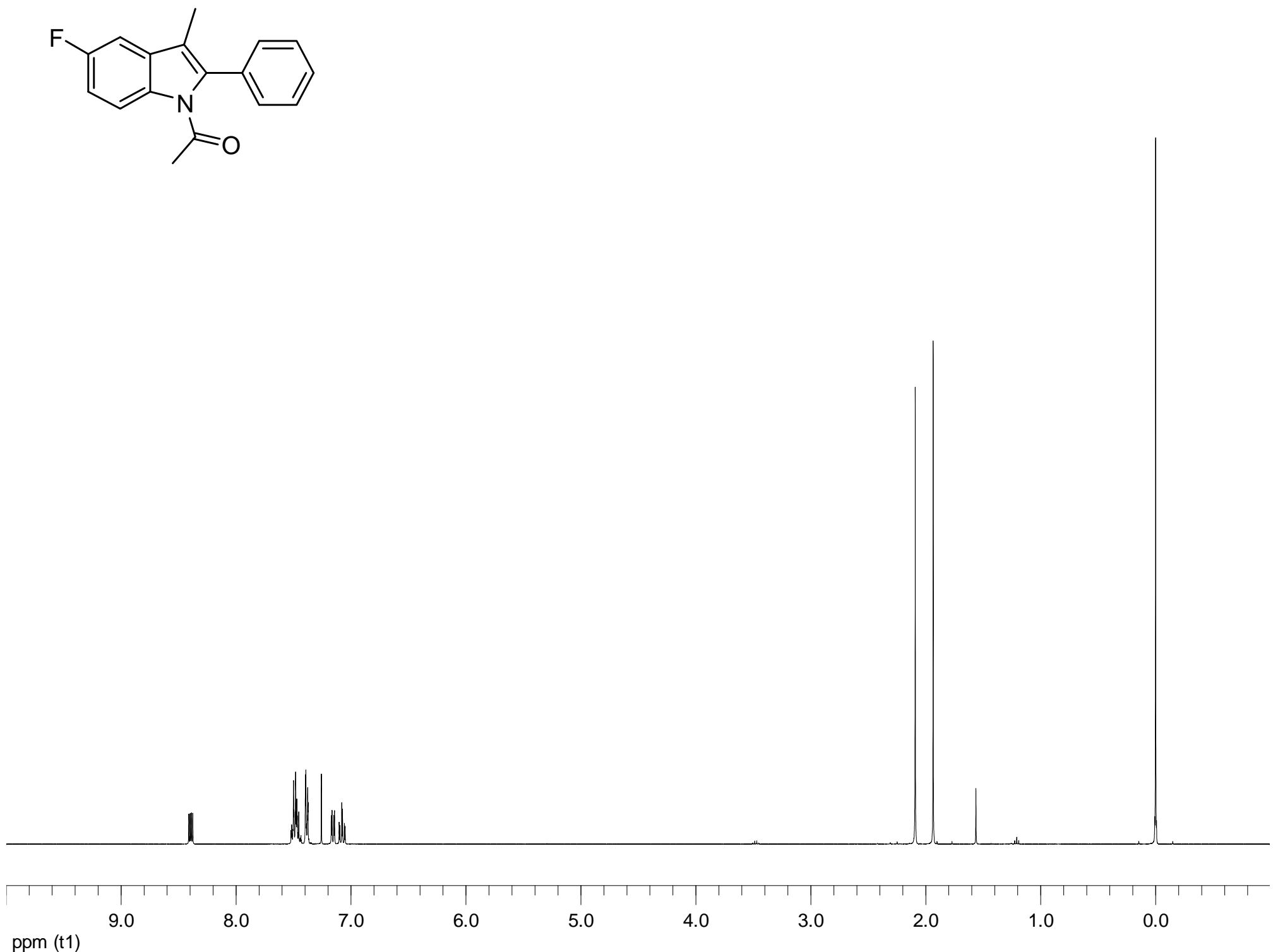

ppm (t1) 

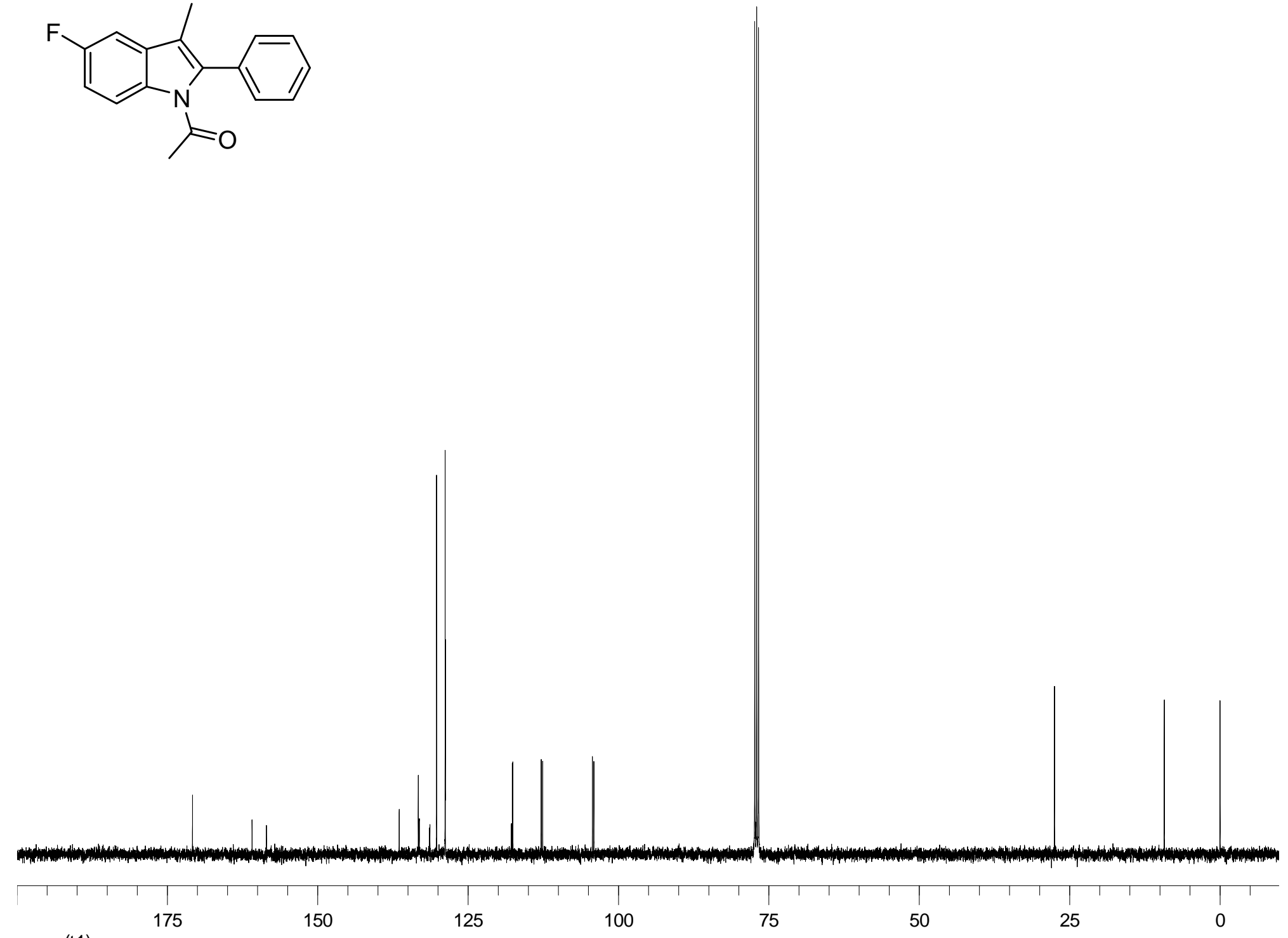

ppm (t1) 

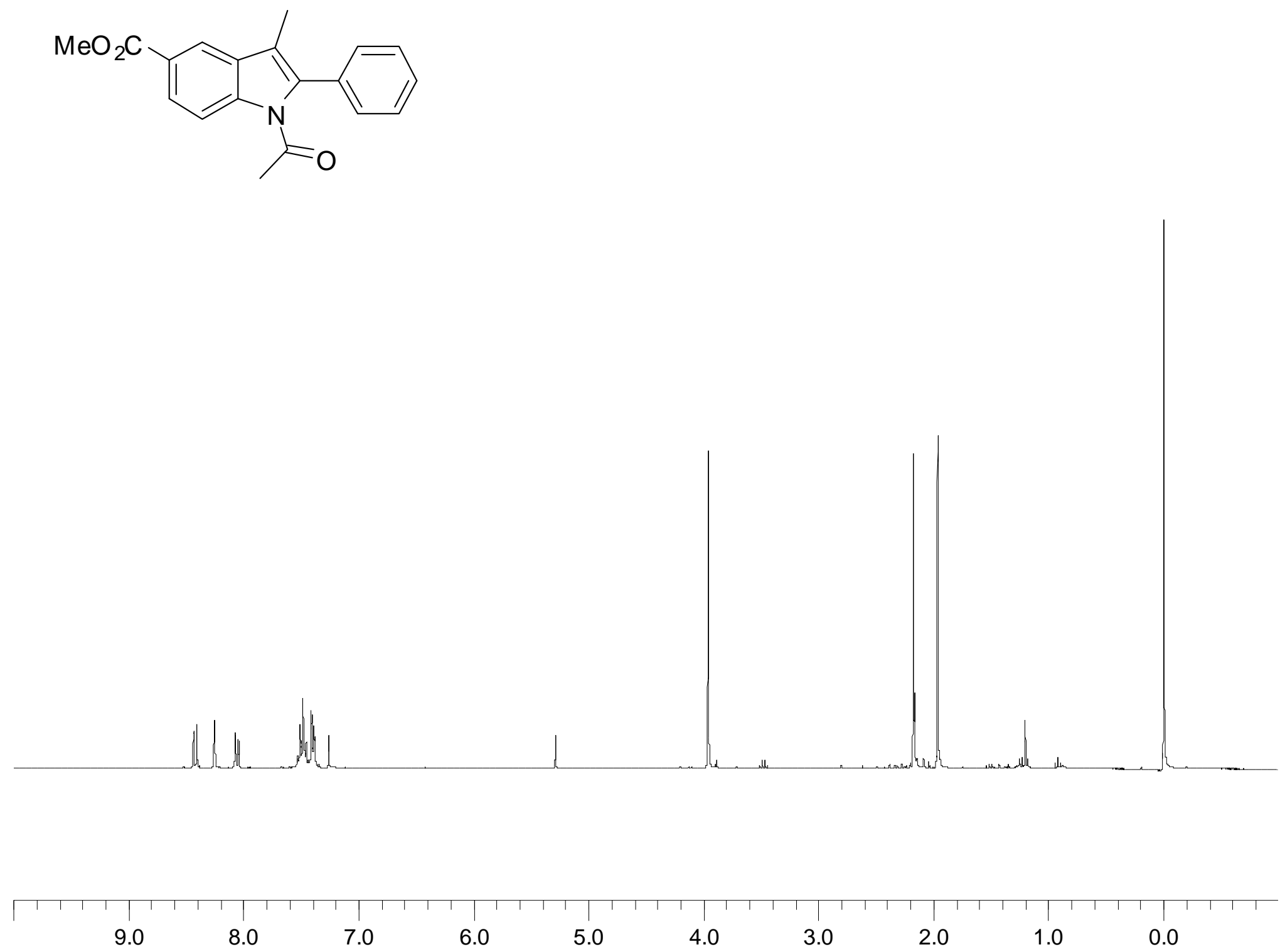

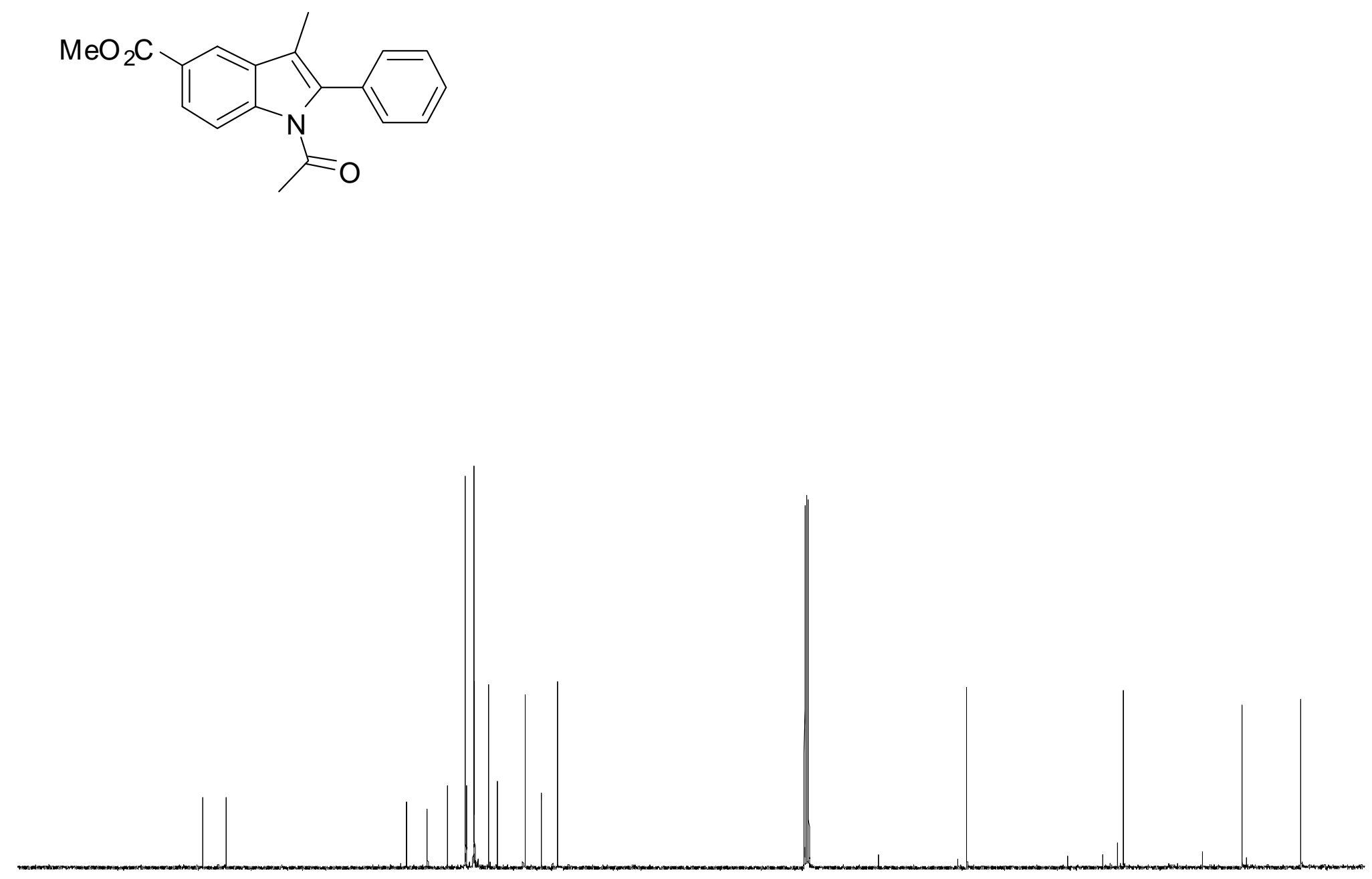

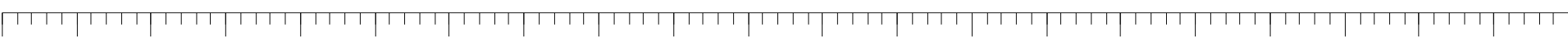

$\begin{array}{llllllllllllllllllll}190 & 180 & 170 & 160 & 150 & 140 & 130 & 120 & 110 & 100 & 90 & 80 & 70 & 60 & 50 & 40 & 30 & 20 & 10 & 0\end{array}$ 

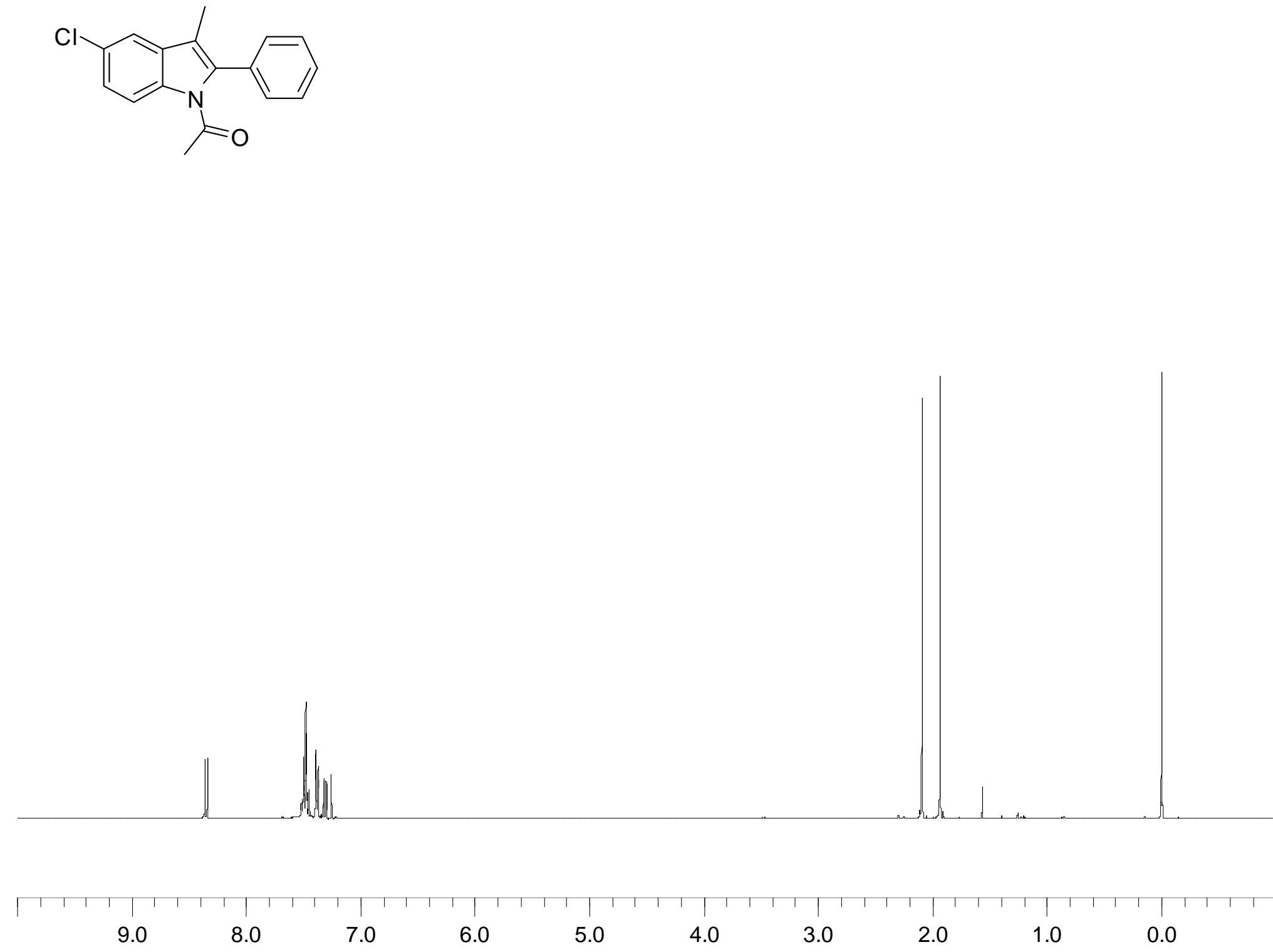

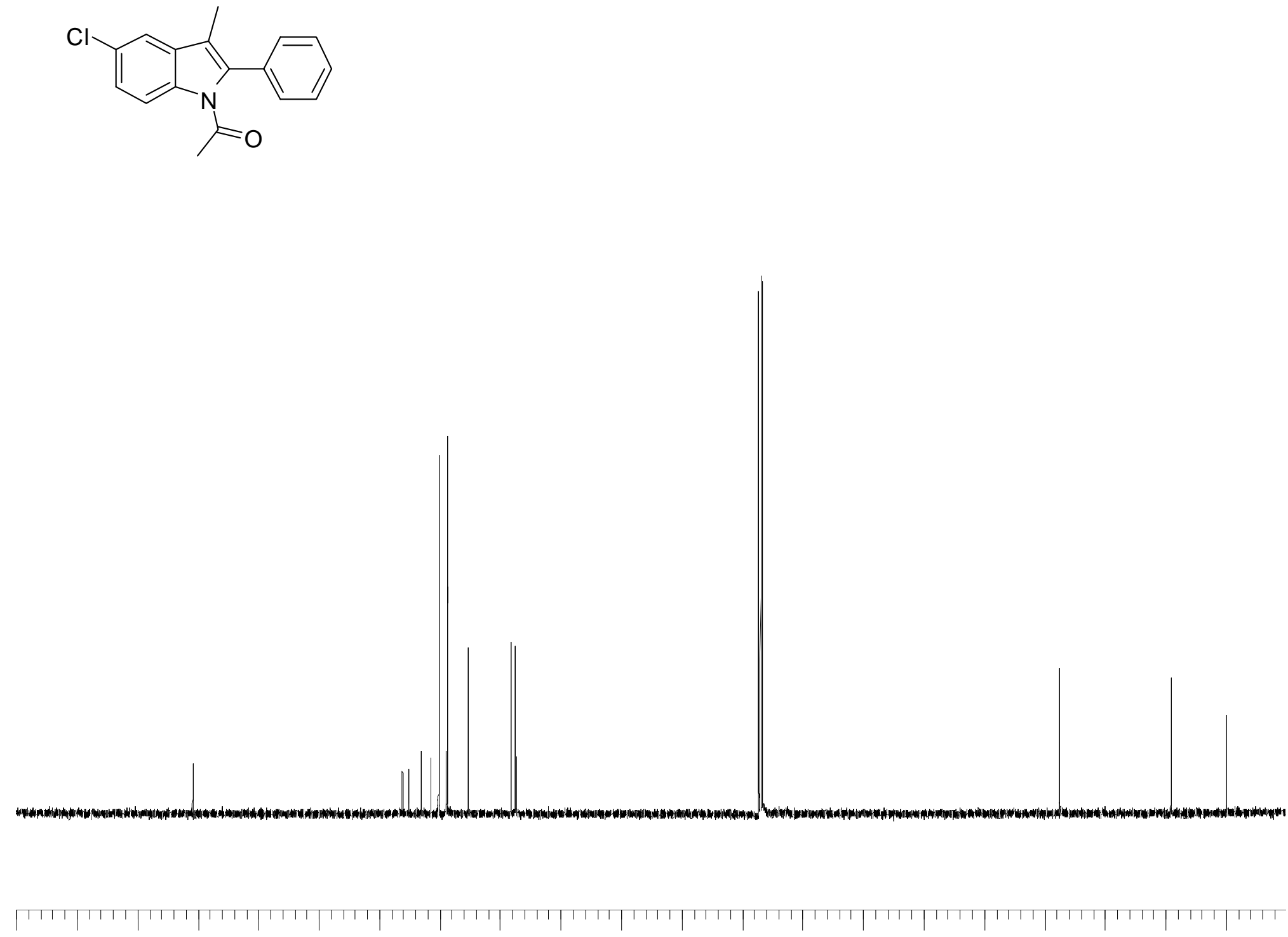

$\begin{array}{llllllllllllllllllll}190 & 180 & 170 & 160 & 150 & 140 & 130 & 120 & 110 & 100 & 90 & 80 & 70 & 60 & 50 & 40 & 30 & 20 & 10 & 0\end{array}$ 

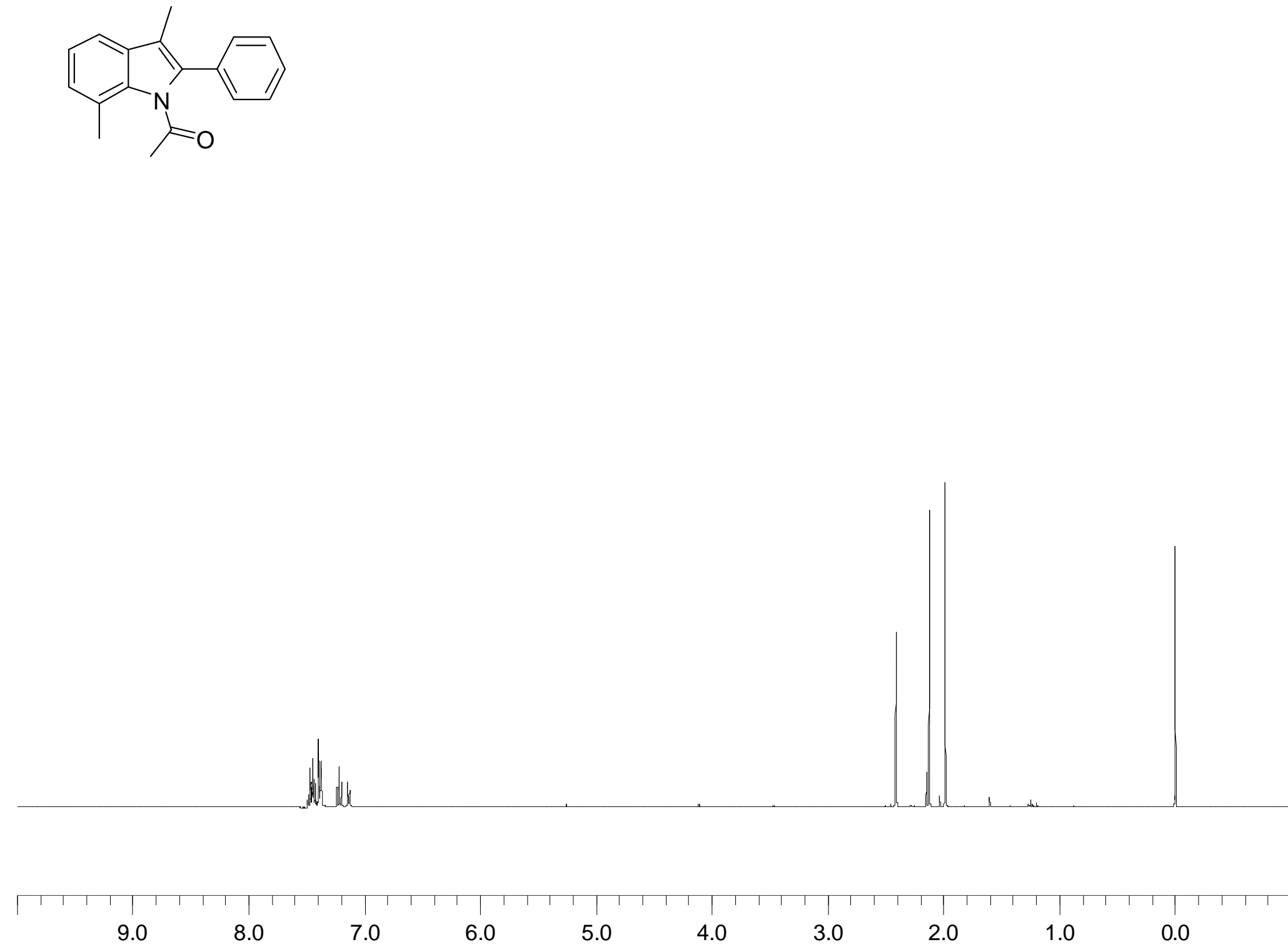

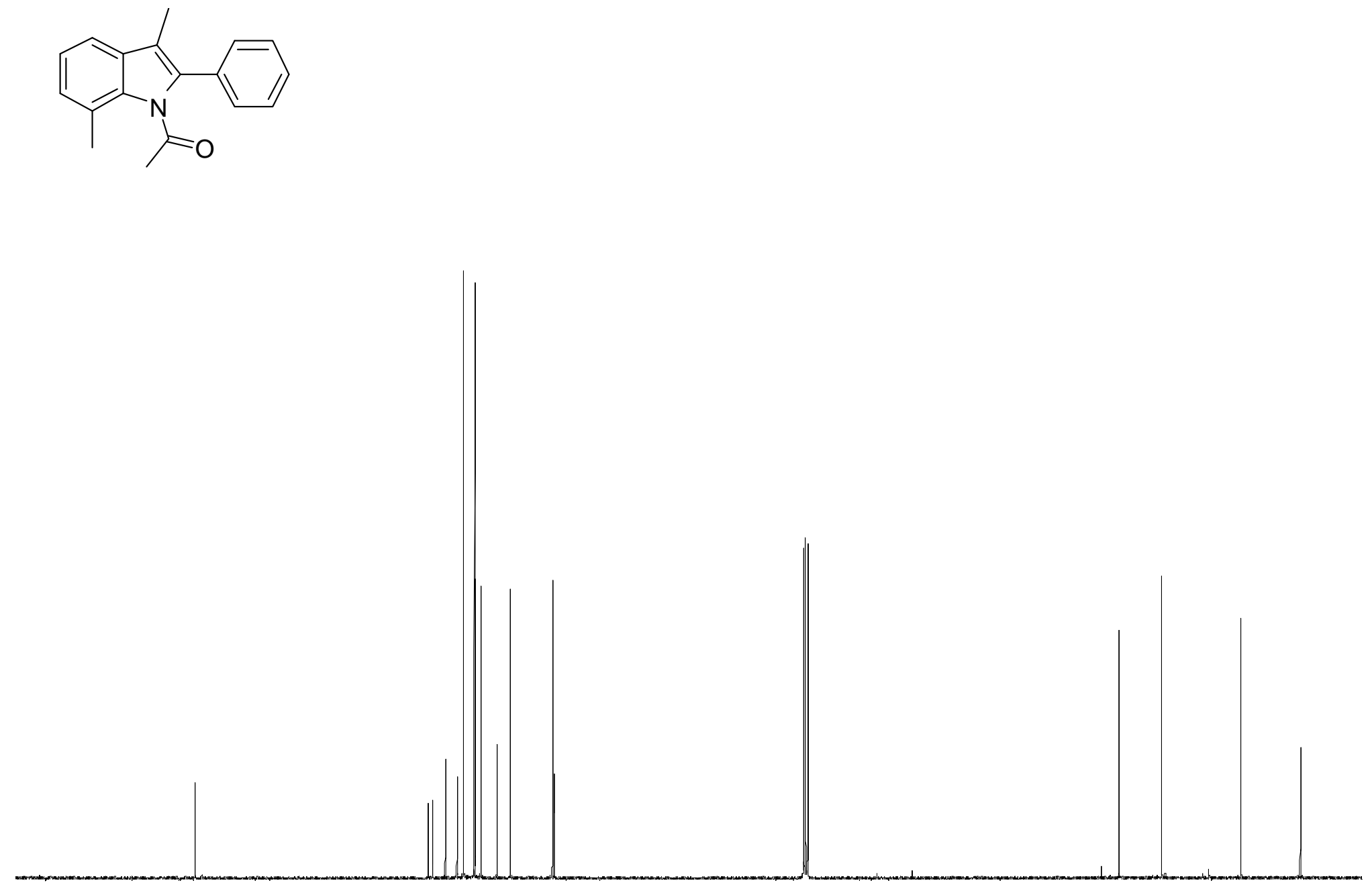

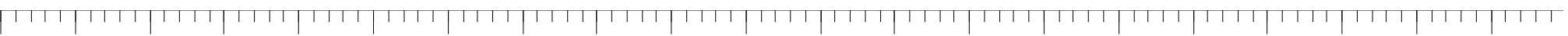

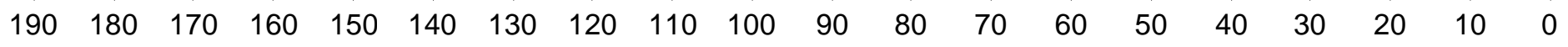



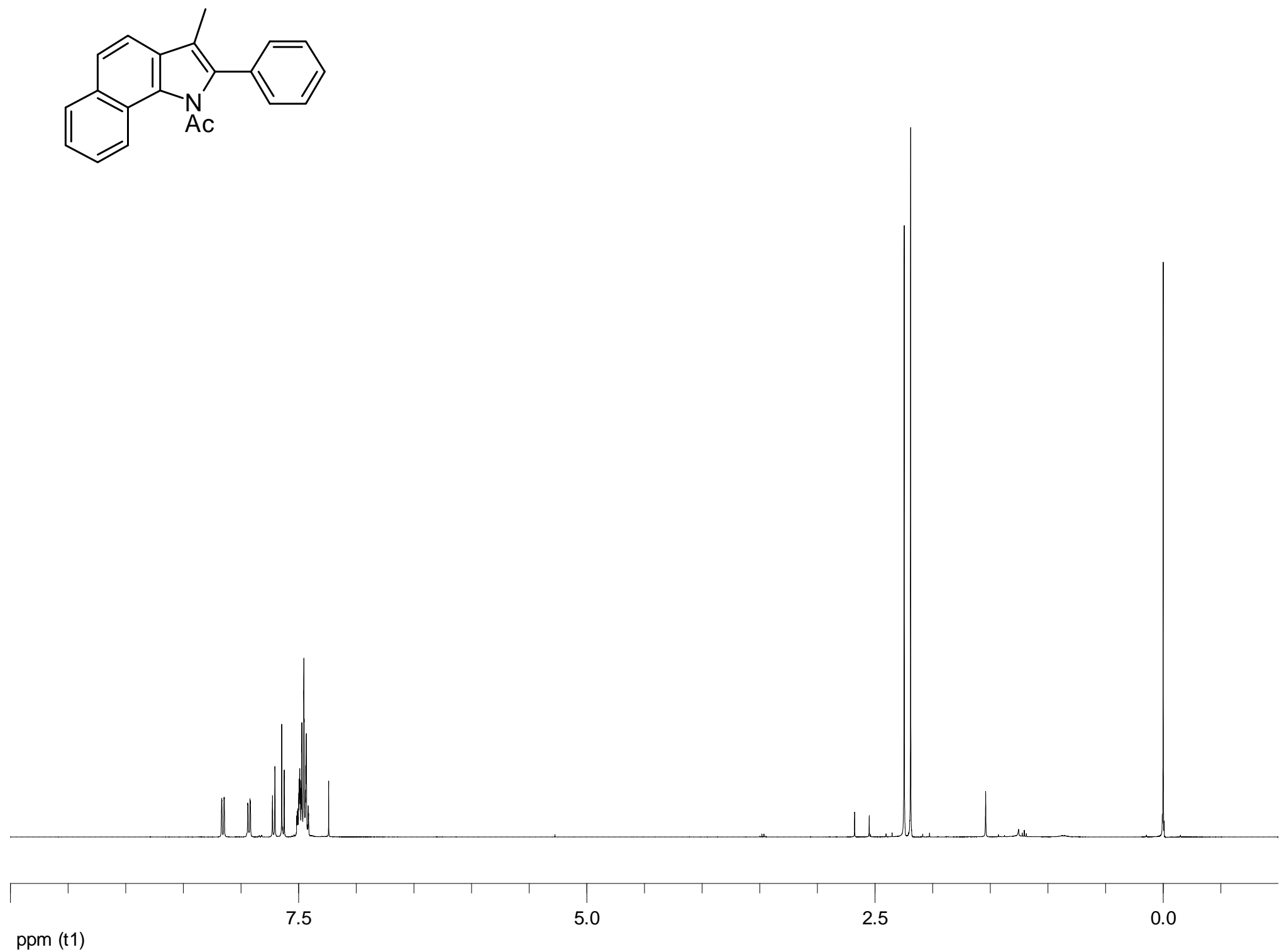

ppm (t1) 

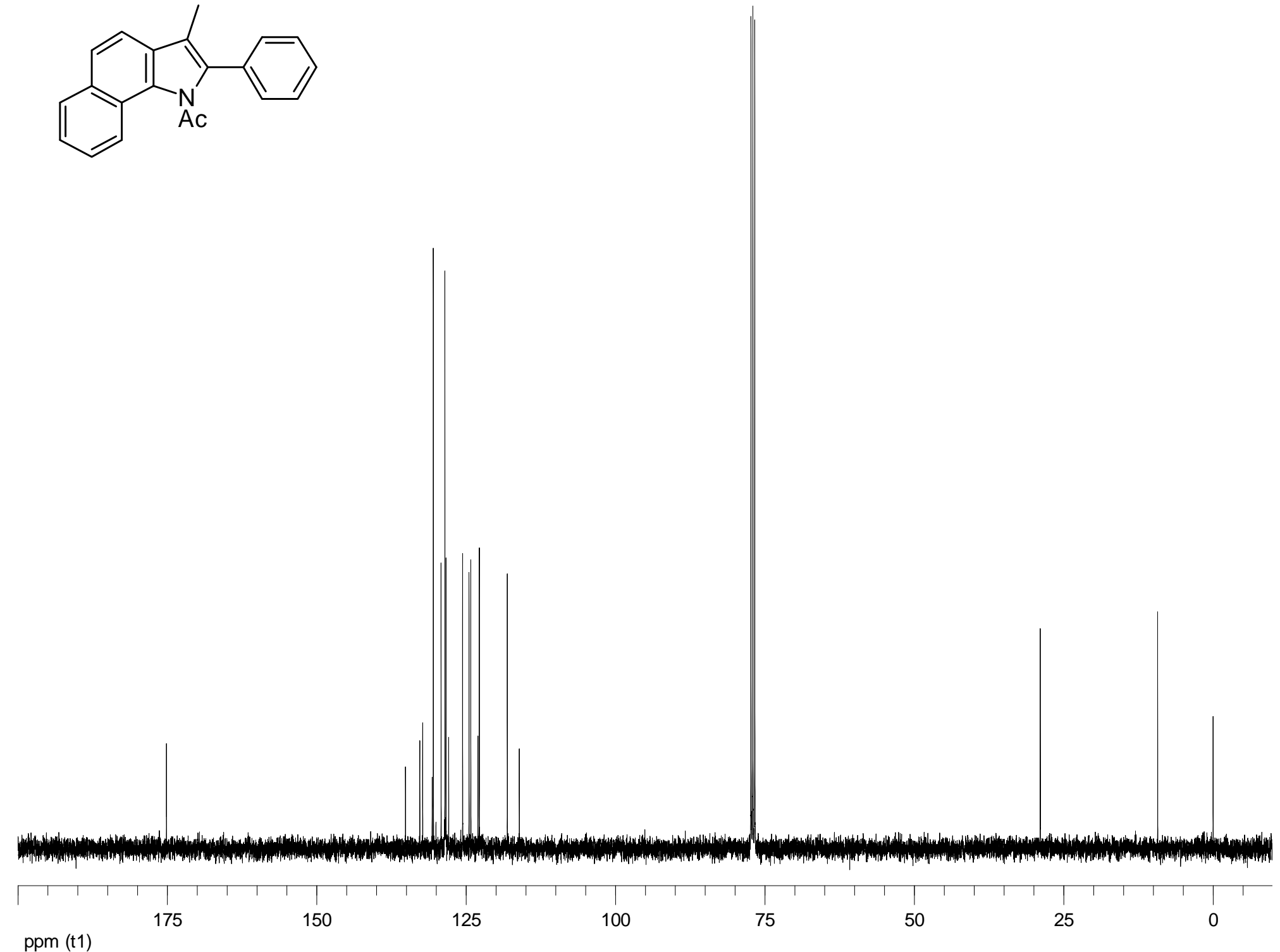

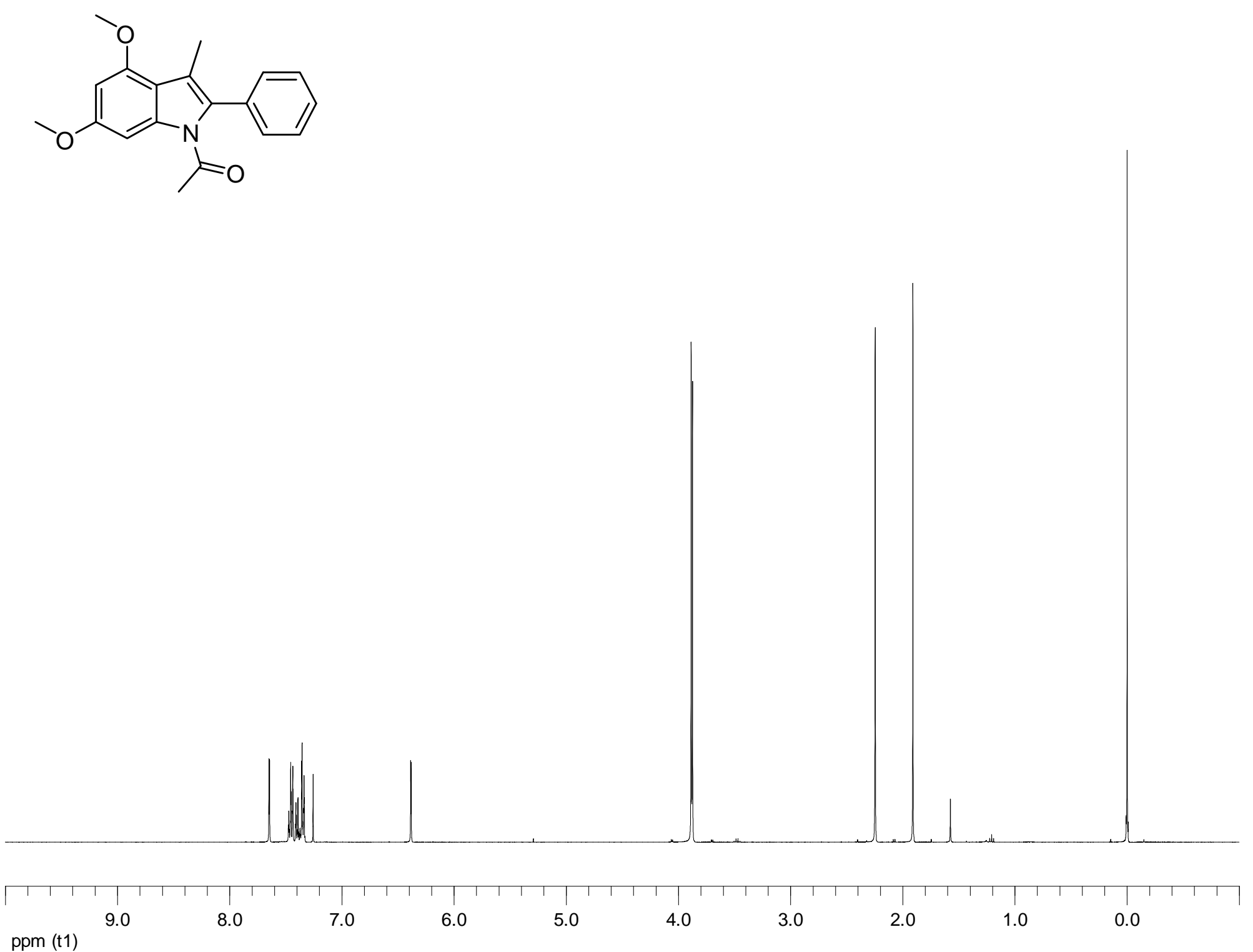

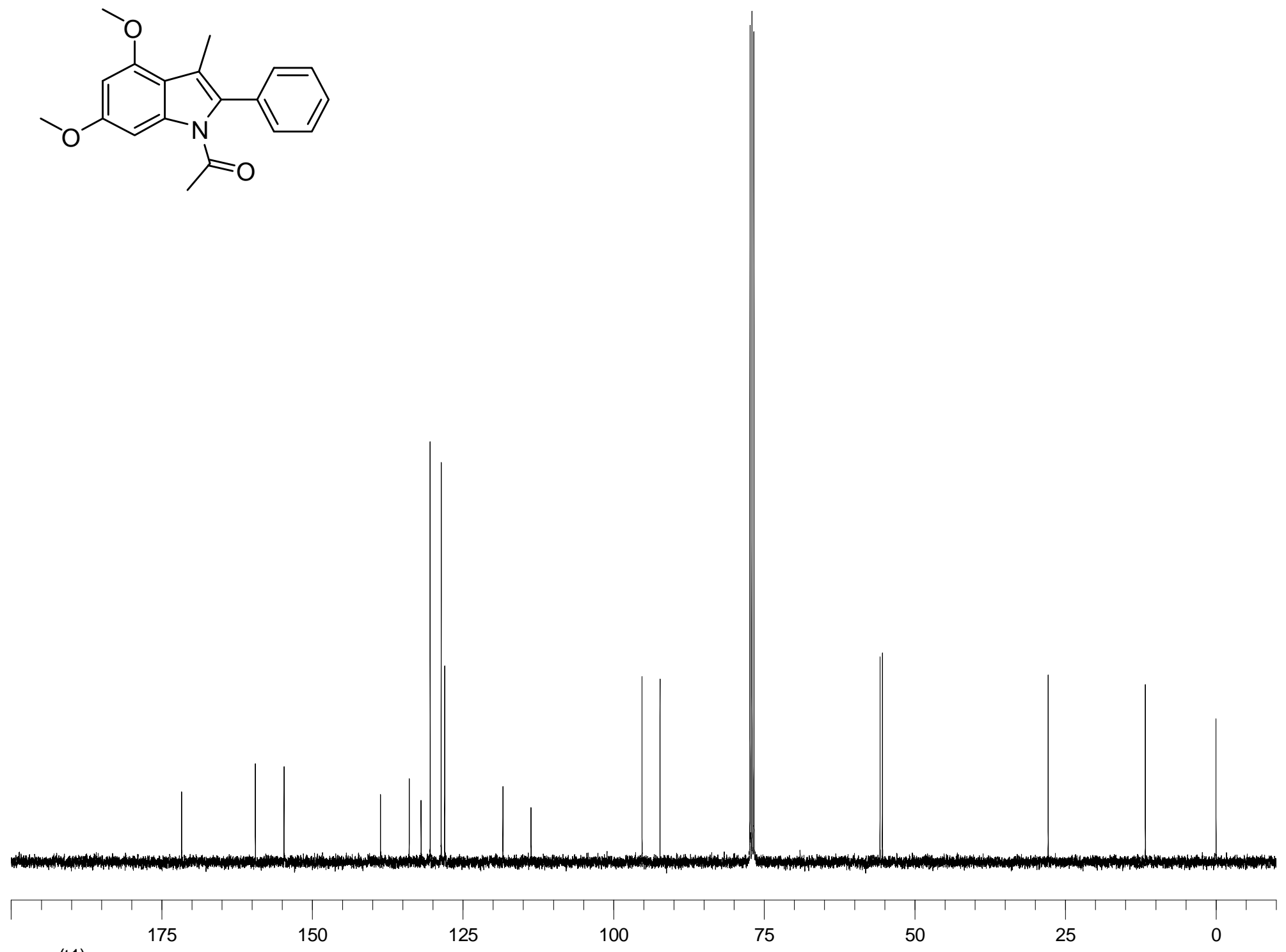

ppm (t1) 

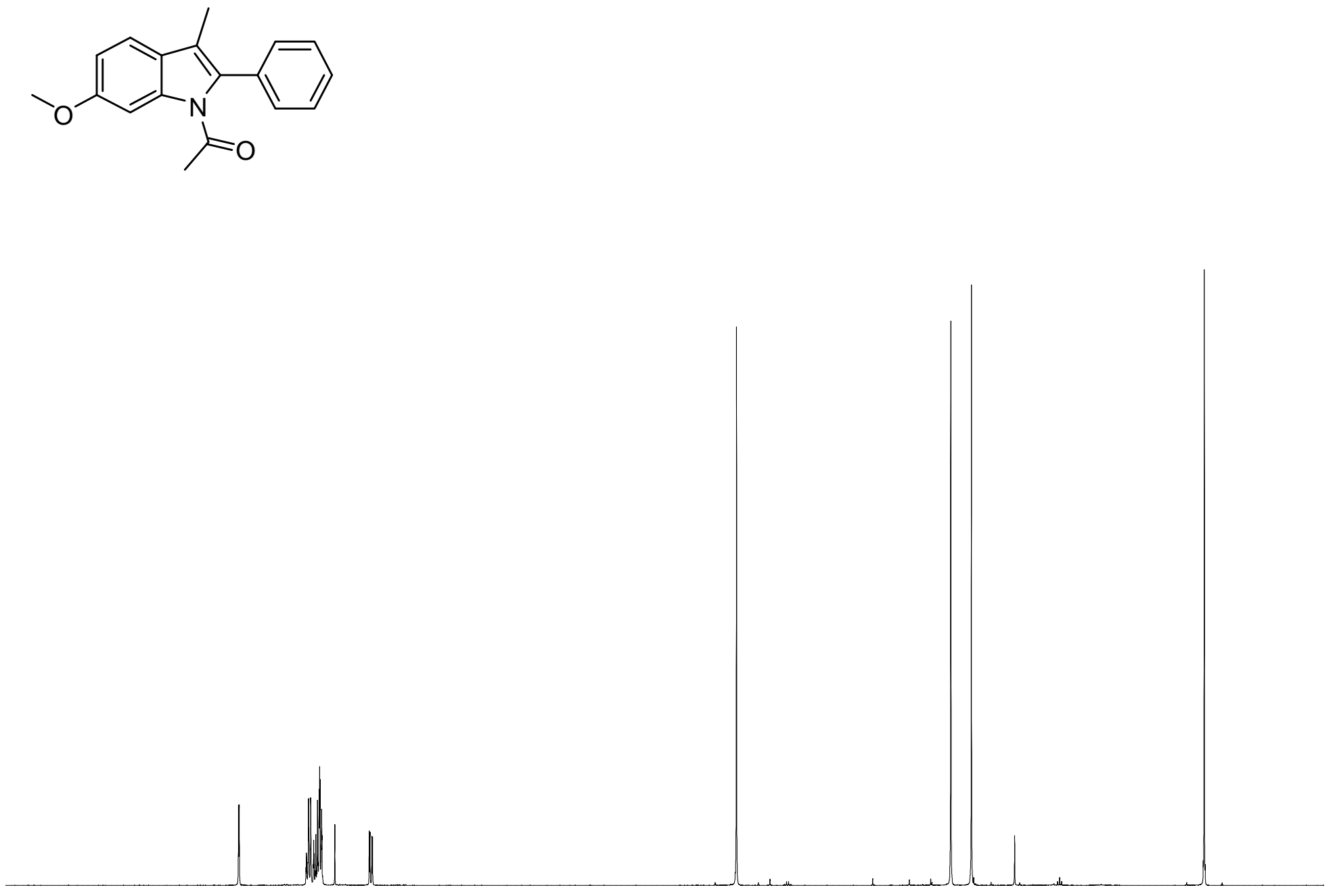

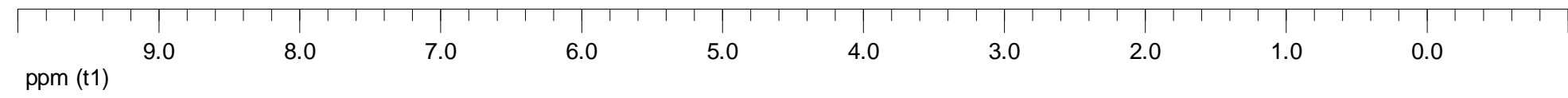



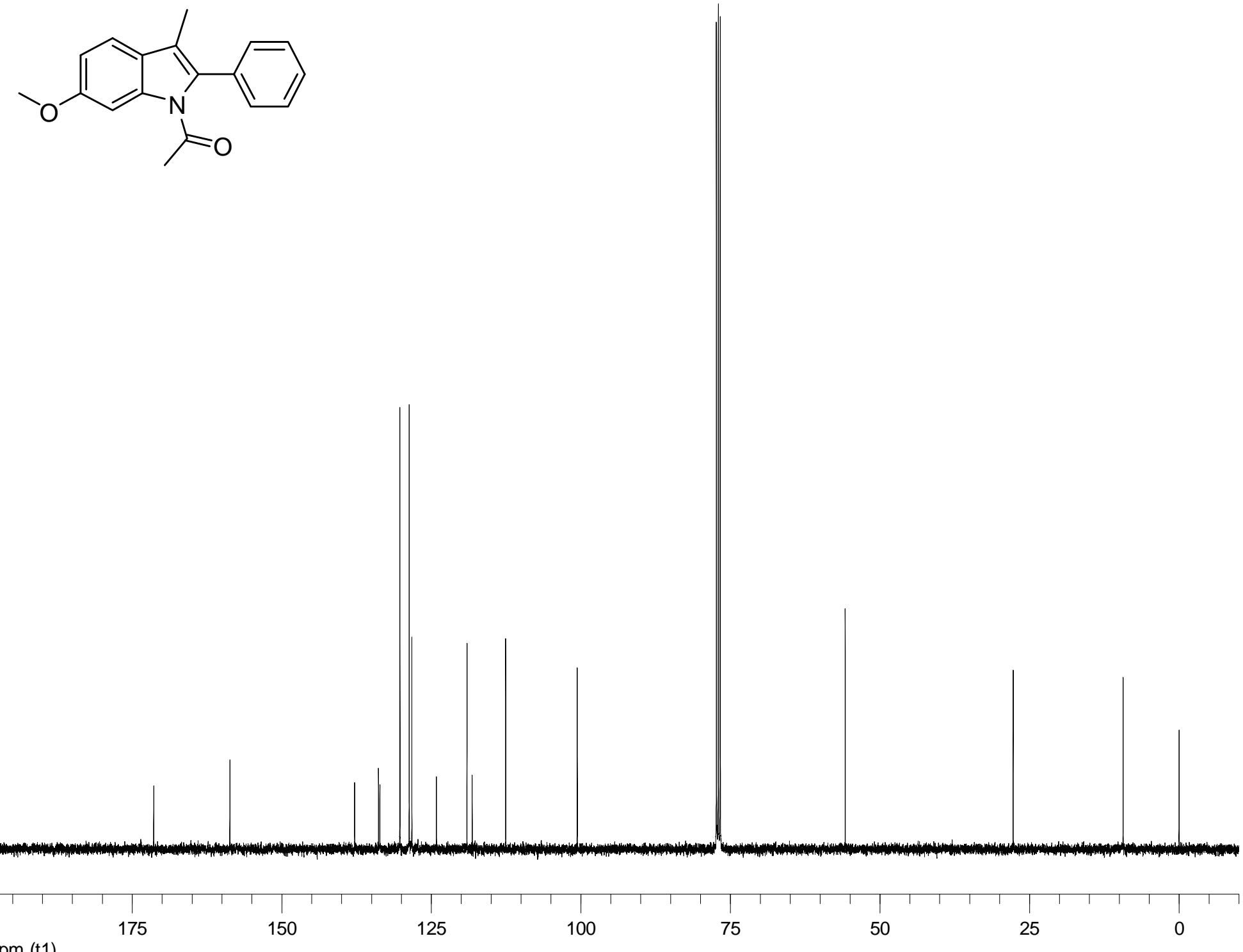

ppm (t1) 

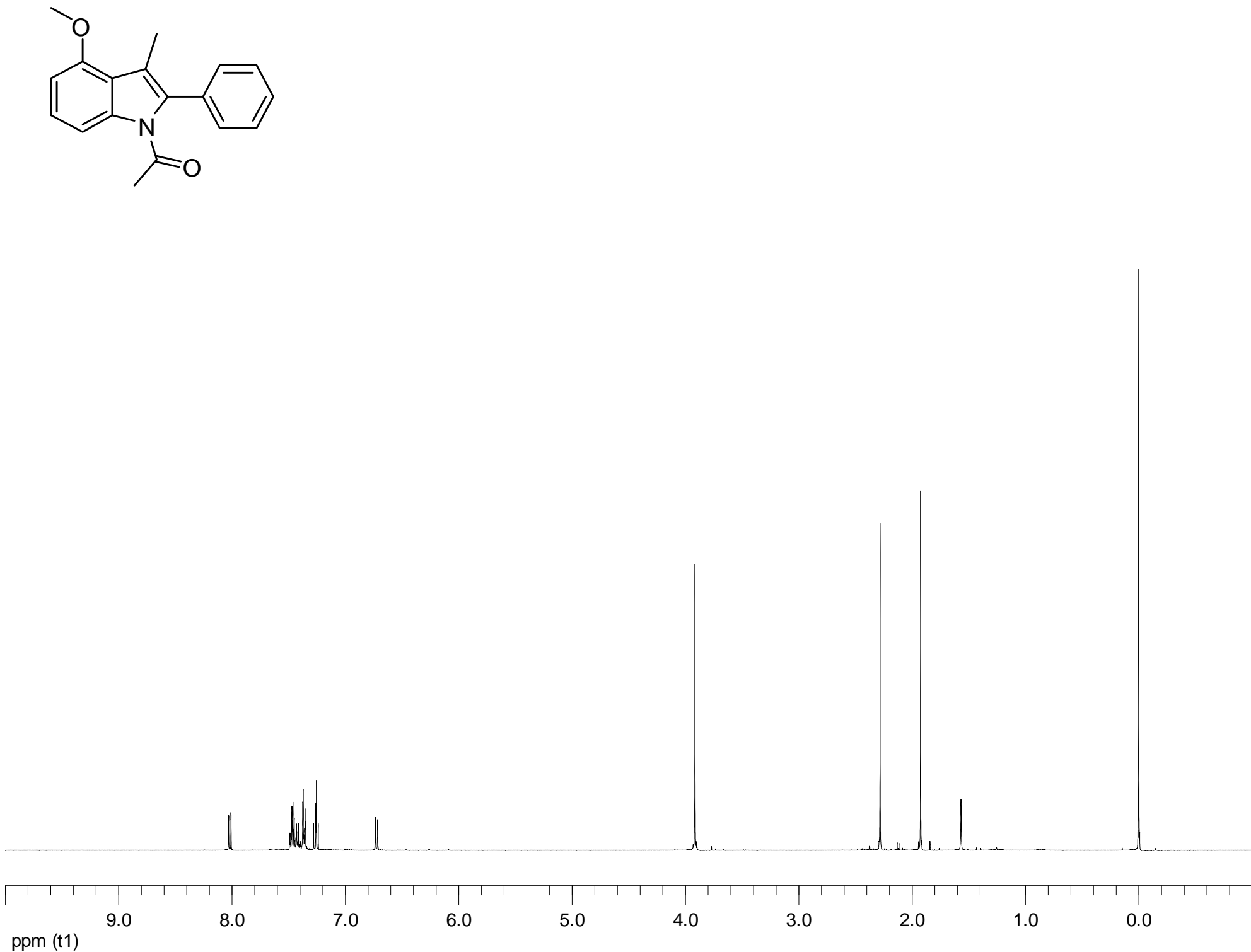


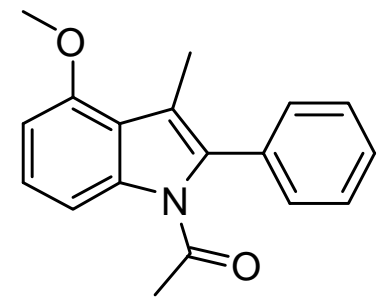

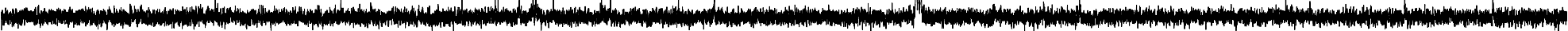

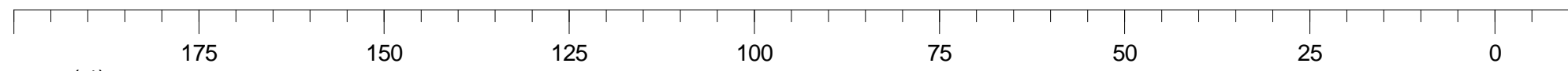

ppm (t1)

100

75

50

5

0 

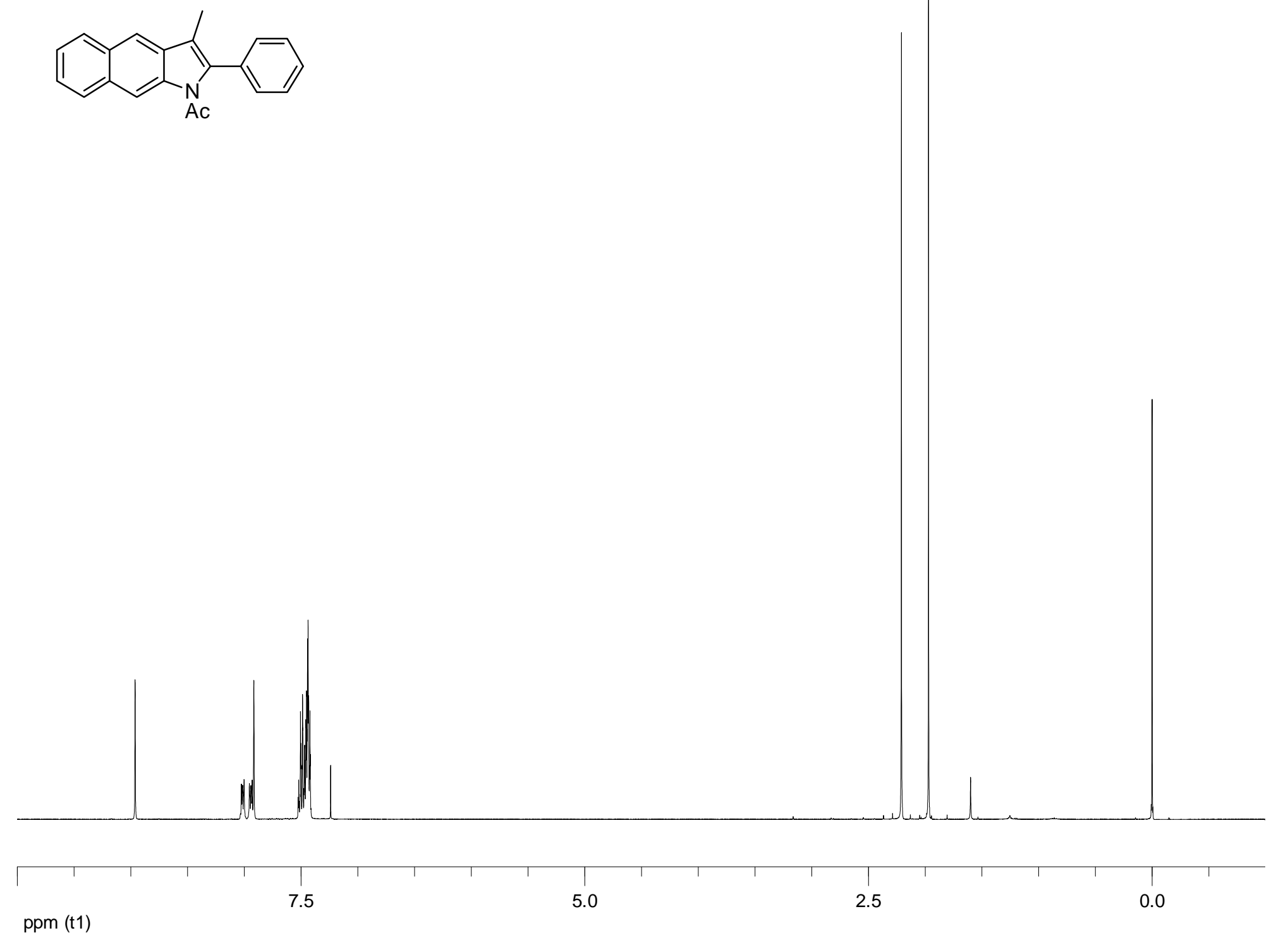

ppm (t1) 


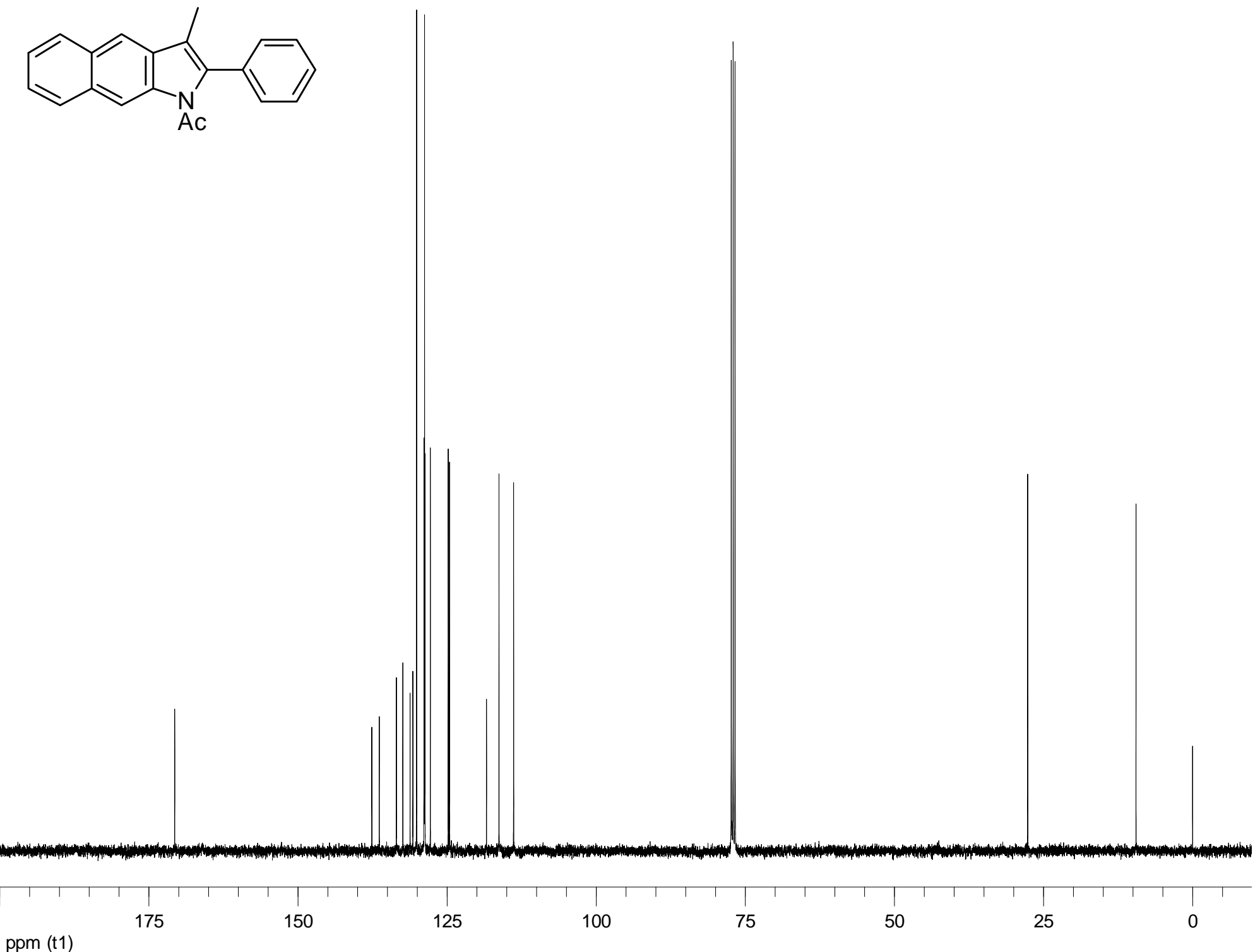



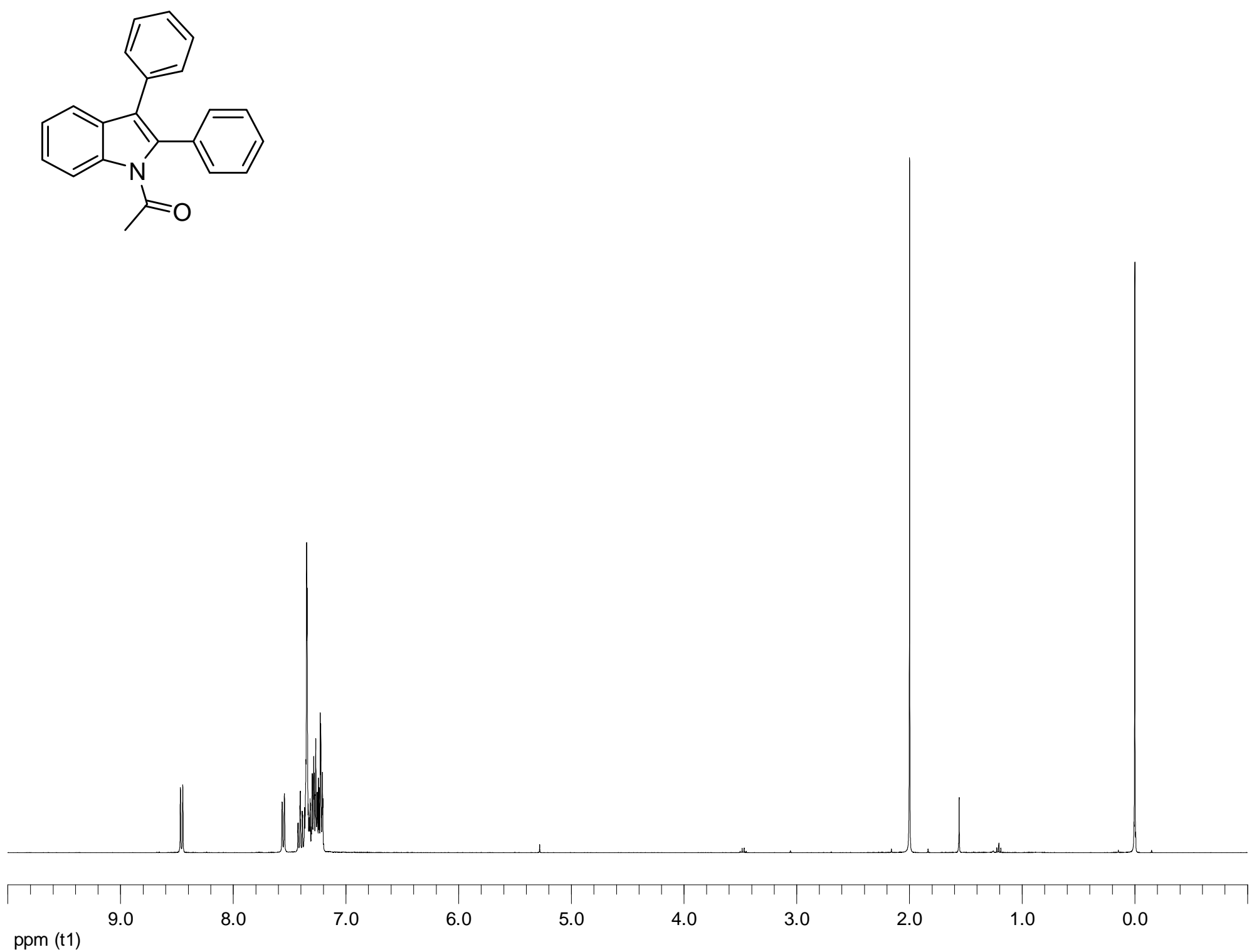

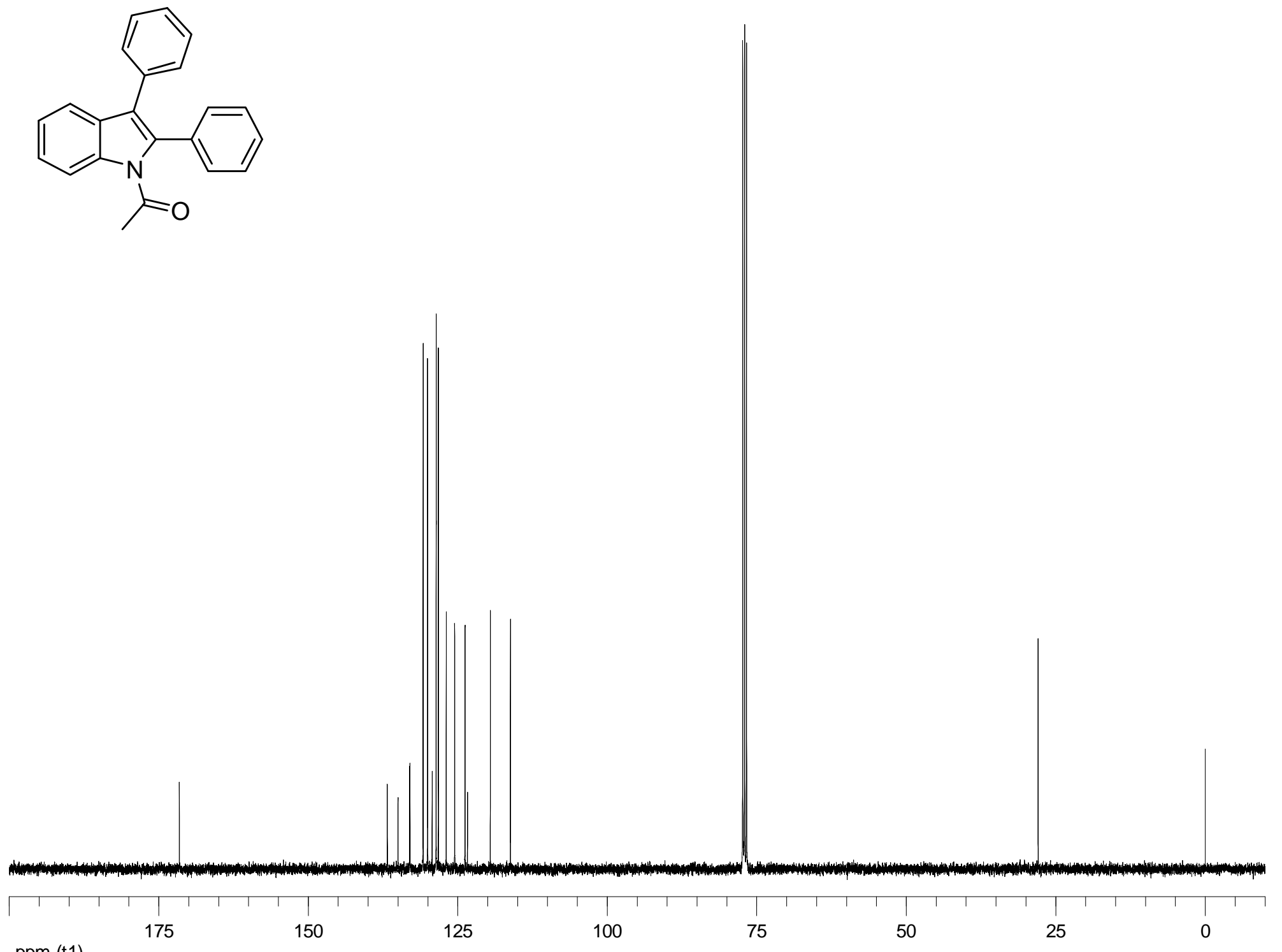

ppm (t1) 

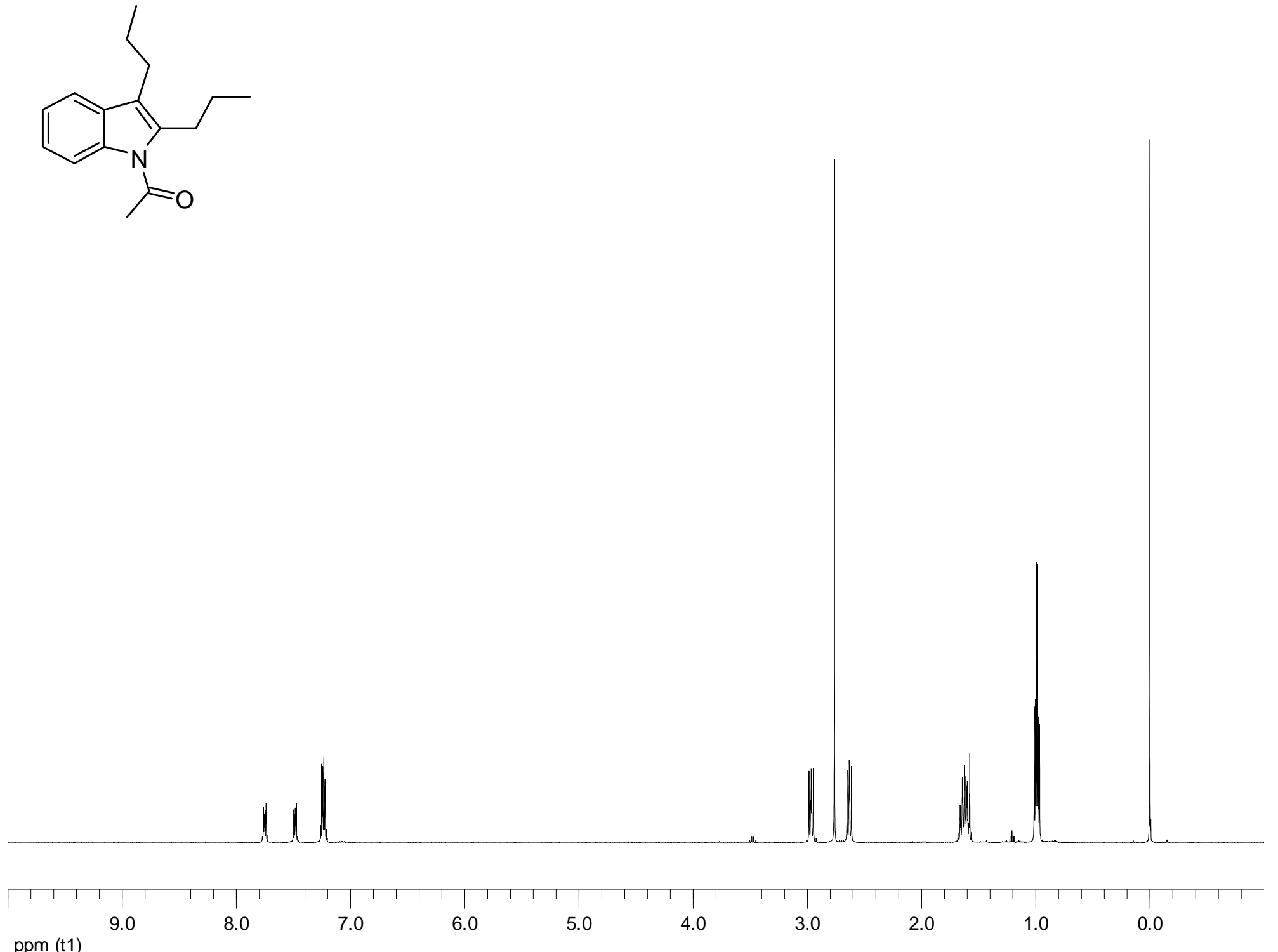

ppm (t1) 

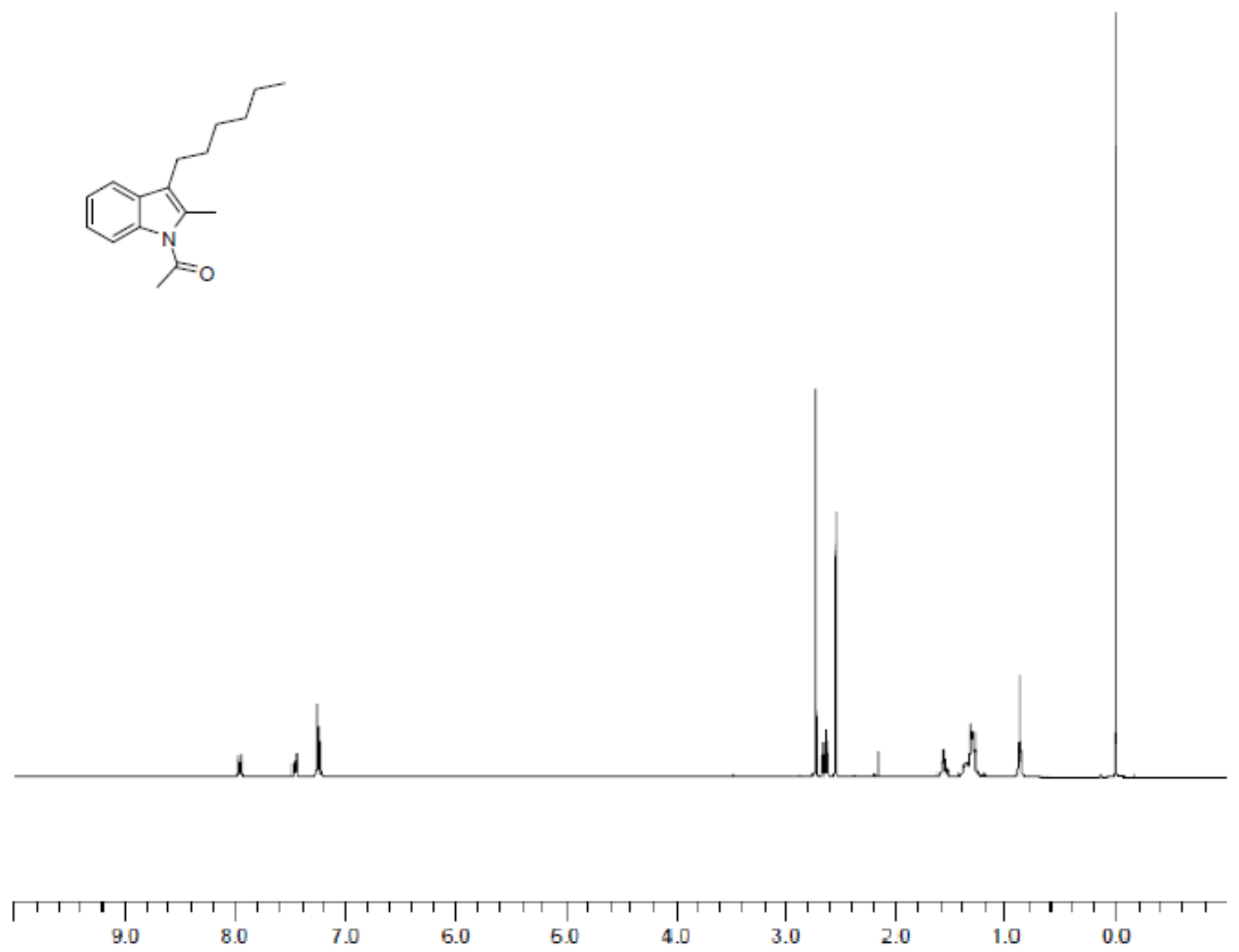

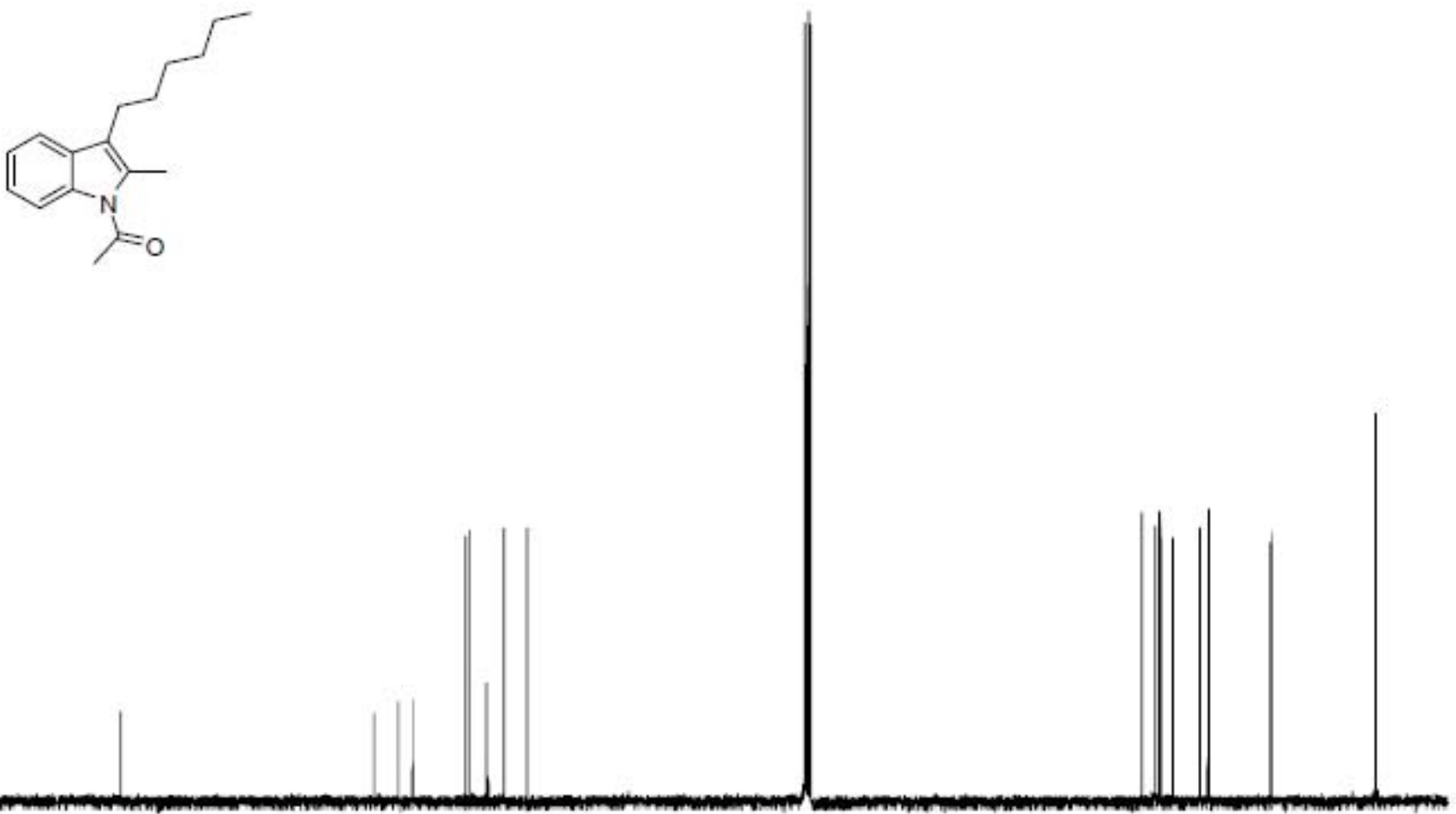

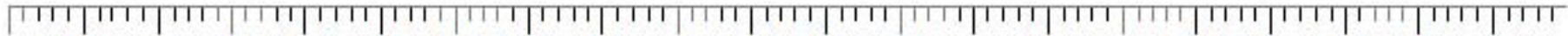
$\begin{array}{llllllllllllllllllll}190 & 180 & 170 & 160 & 150 & 140 & 130 & 120 & 110 & 100 & 90 & 80 & 70 & 60 & 50 & 40 & 30 & 20 & 10 & 0\end{array}$ 
S65

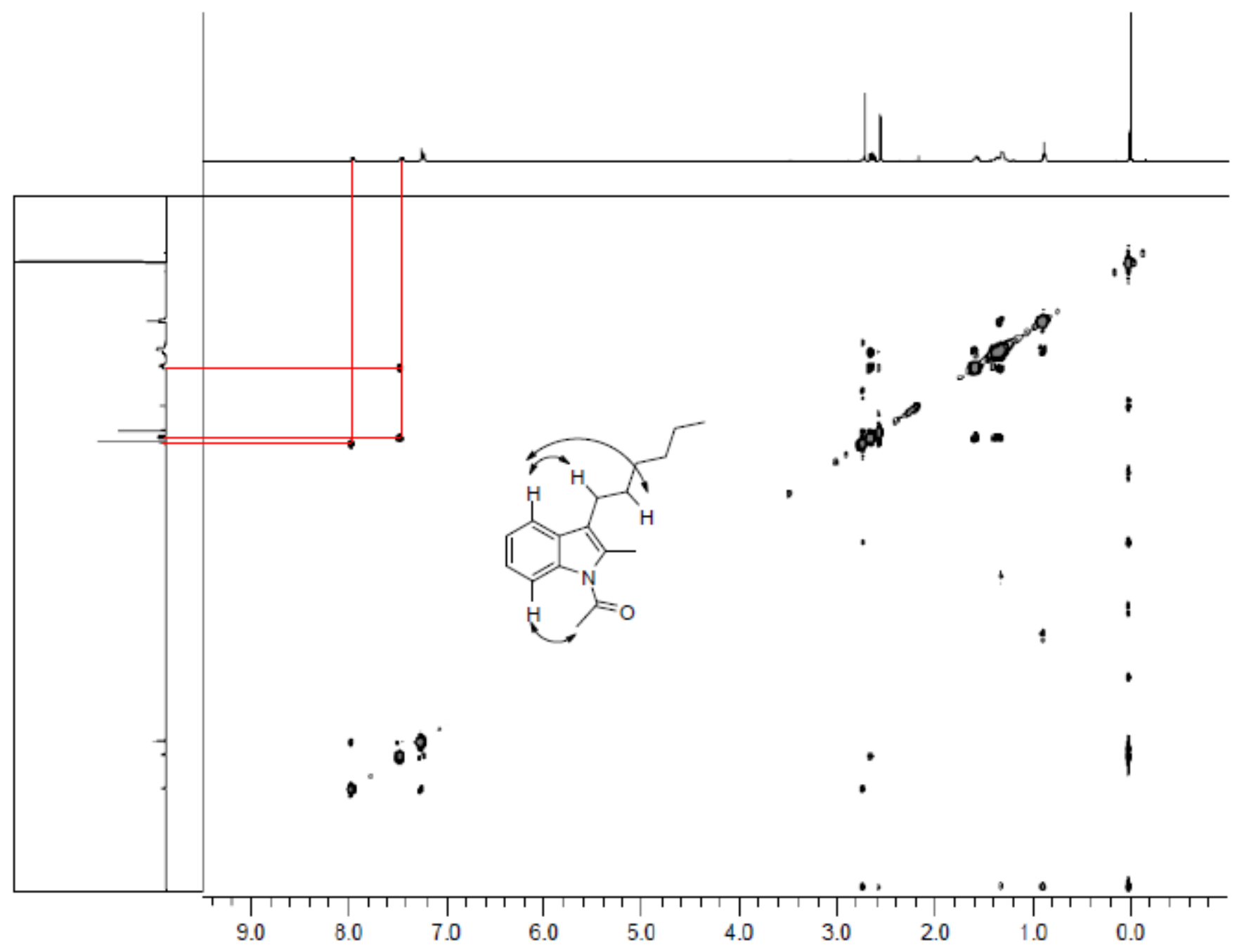




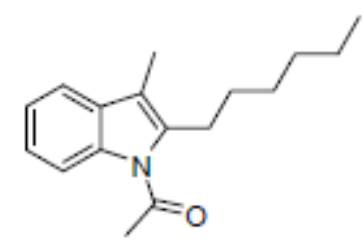

1

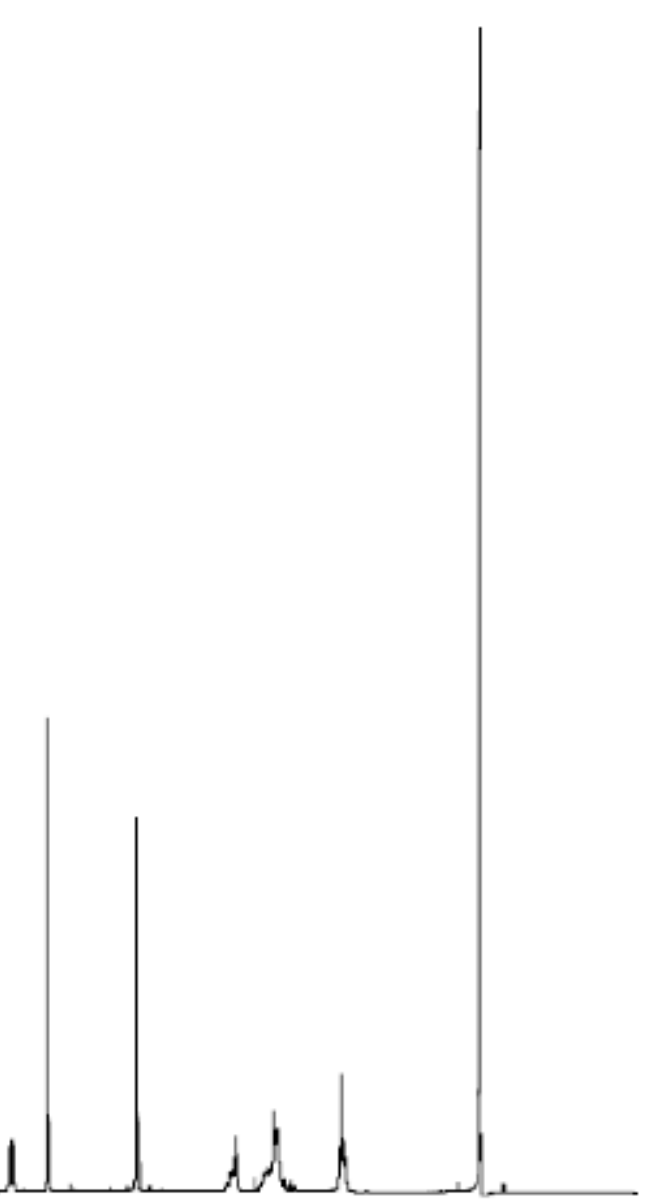

\begin{tabular}{l|l|l|}
\hline 1 & 1
\end{tabular} 

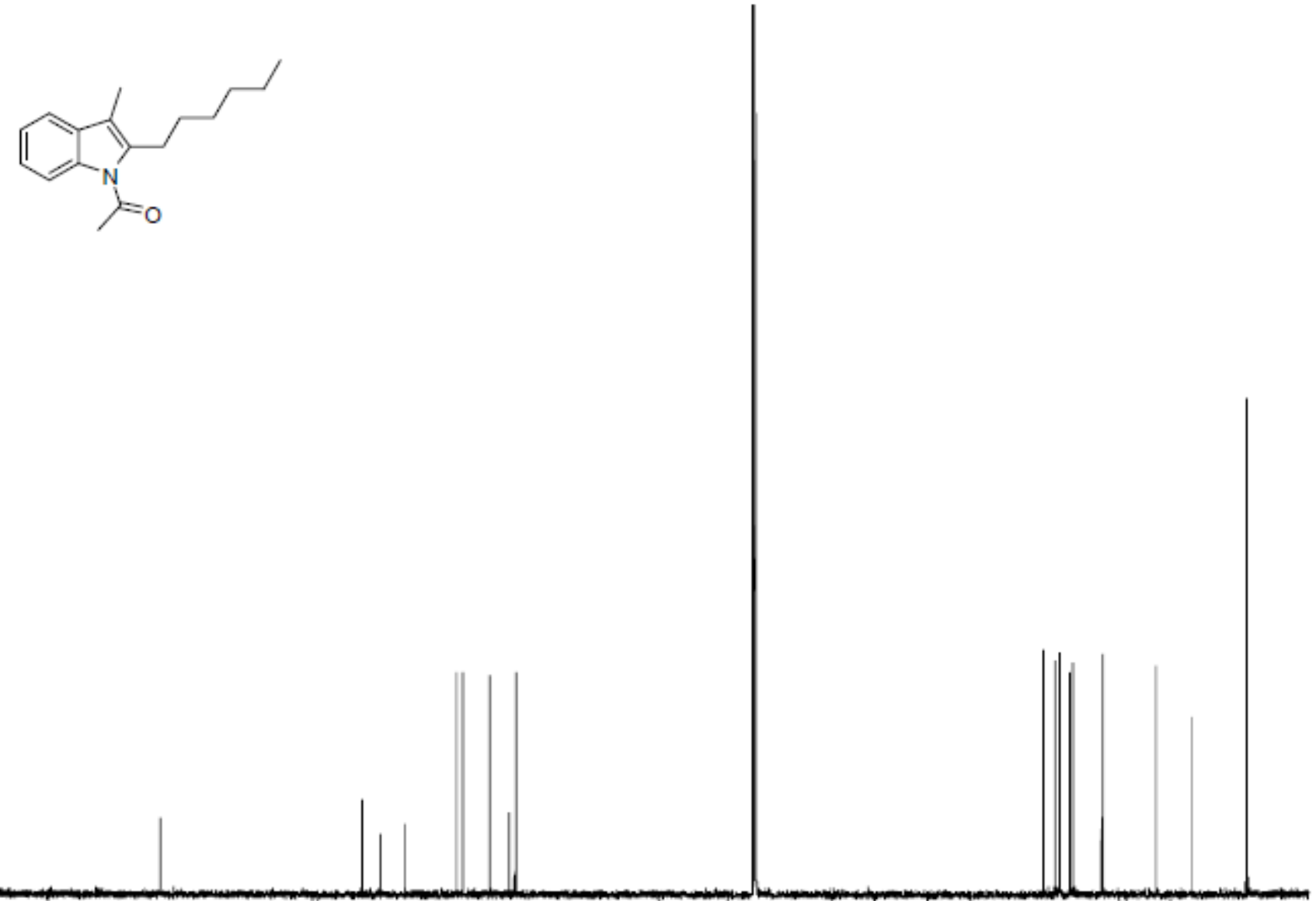

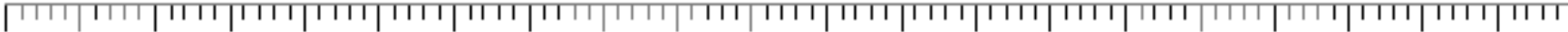
$\begin{array}{llllllllllllllllllll}190 & 180 & 170 & 160 & 150 & 140 & 130 & 120 & 110 & 100 & 90 & 80 & 70 & 60 & 50 & 40 & 30 & 20 & 10 & 0\end{array}$ 


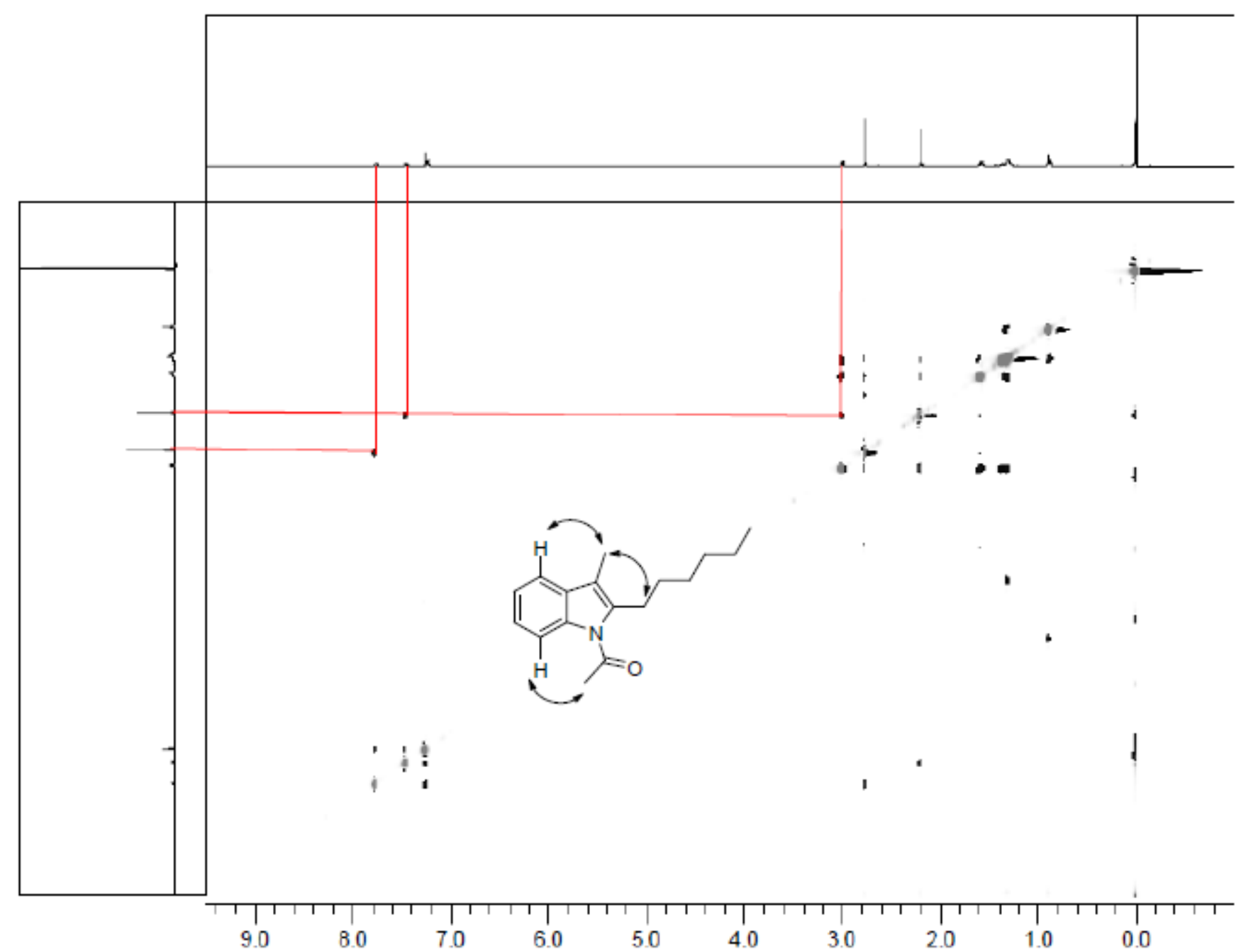



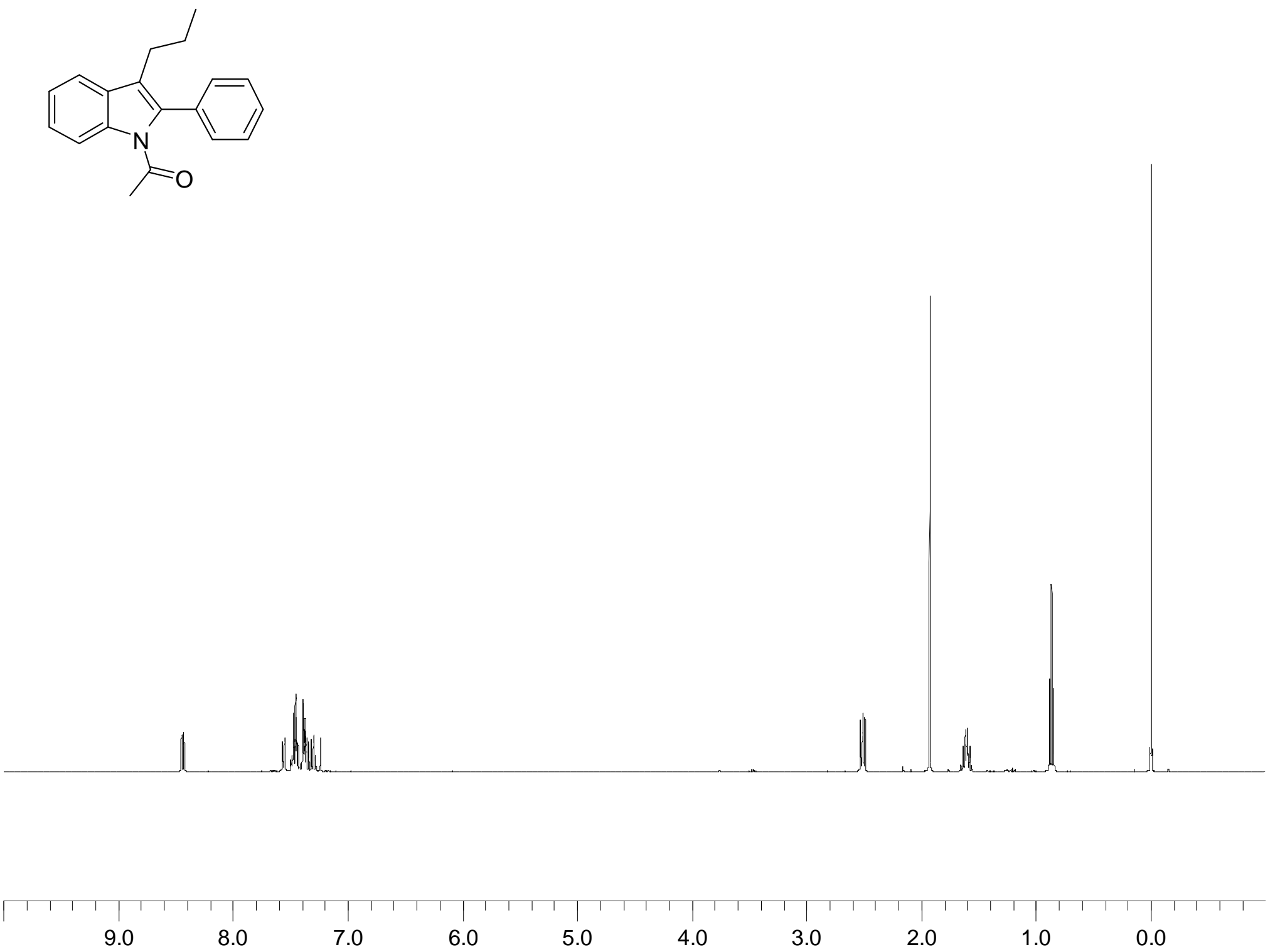

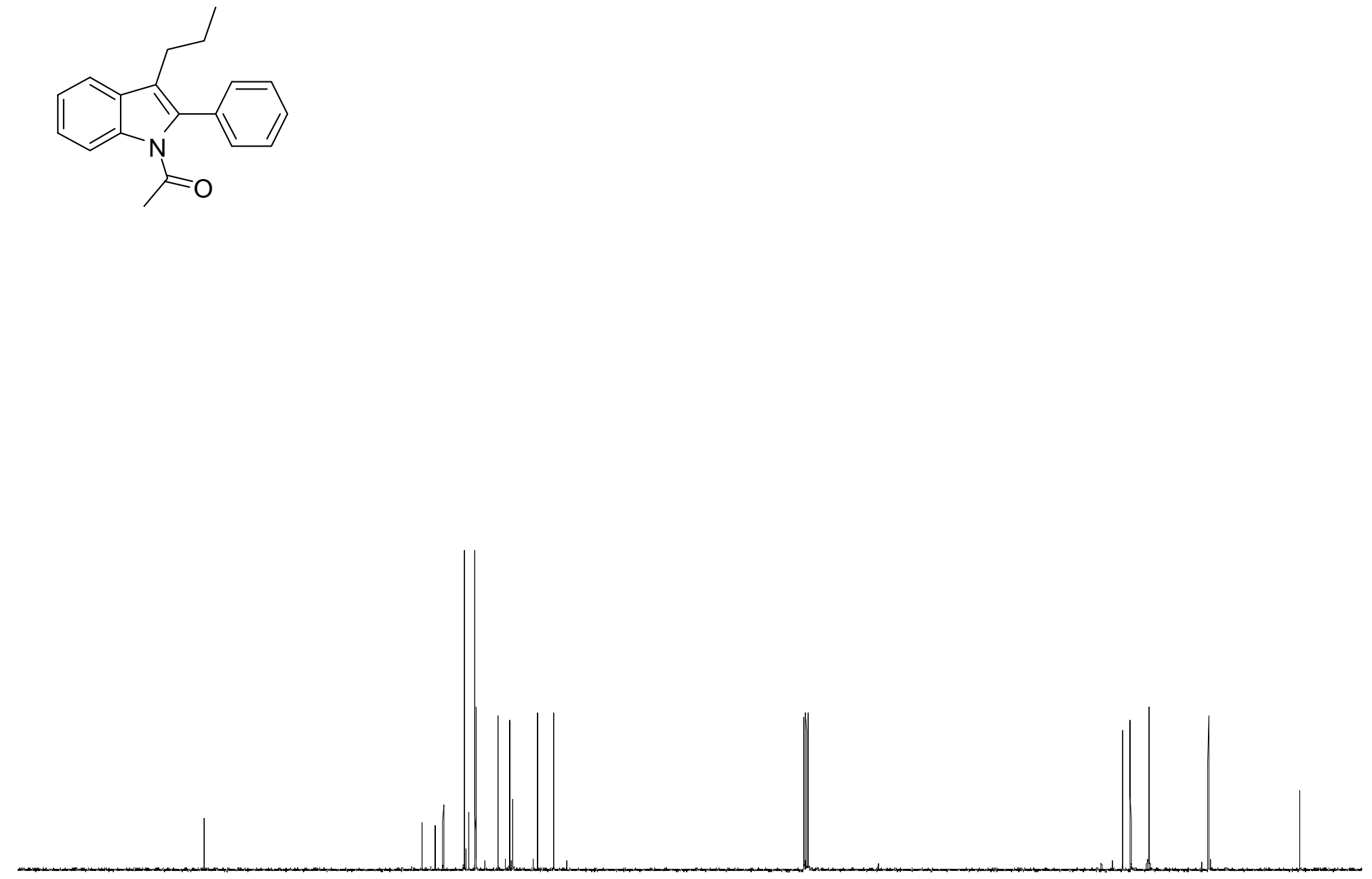

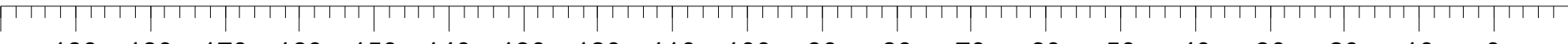

$\begin{array}{llllllllllllllllllll}190 & 180 & 170 & 160 & 150 & 140 & 130 & 120 & 110 & 100 & 90 & 80 & 70 & 60 & 50 & 40 & 30 & 20 & 10 & 0\end{array}$ 

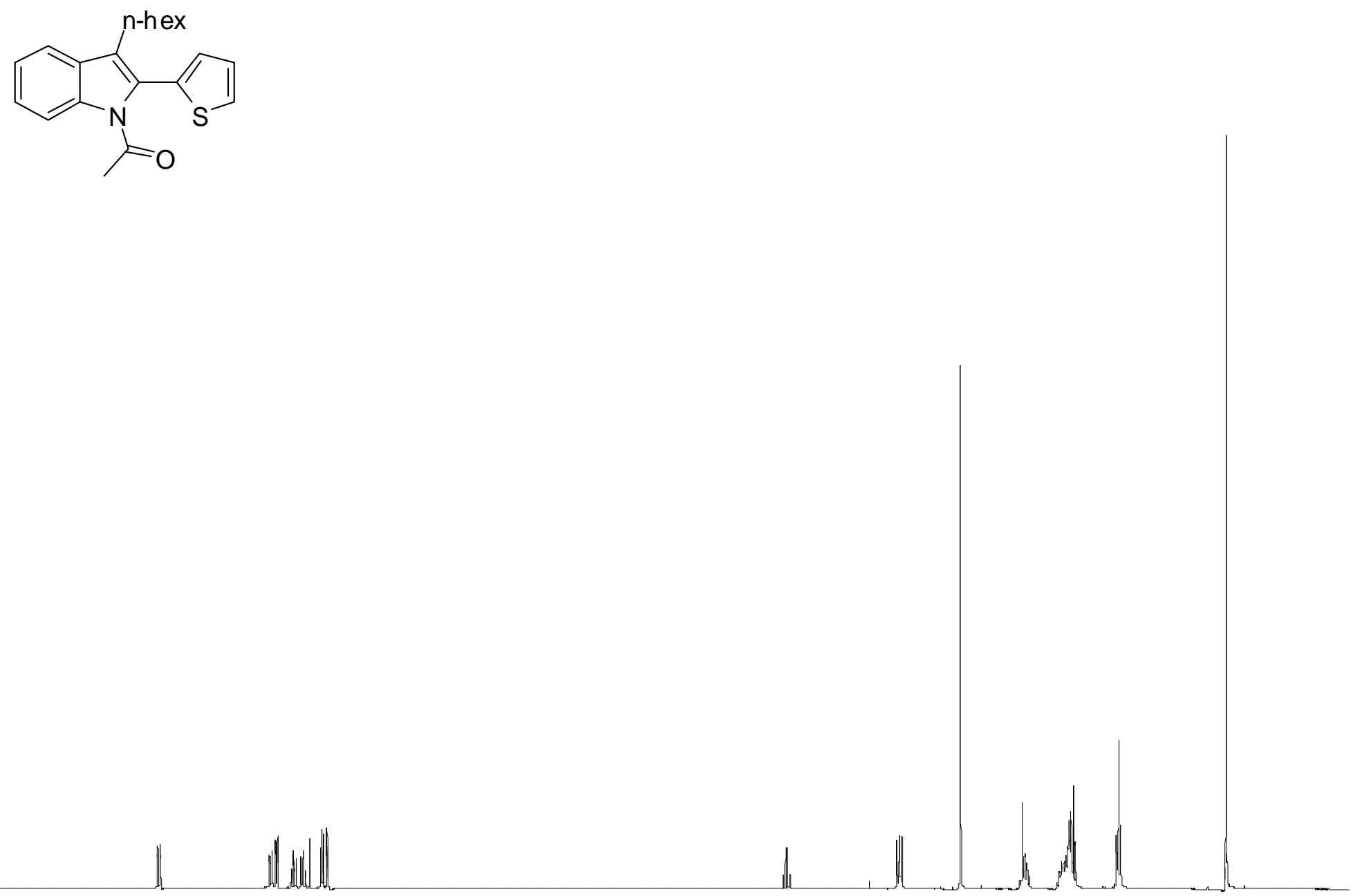

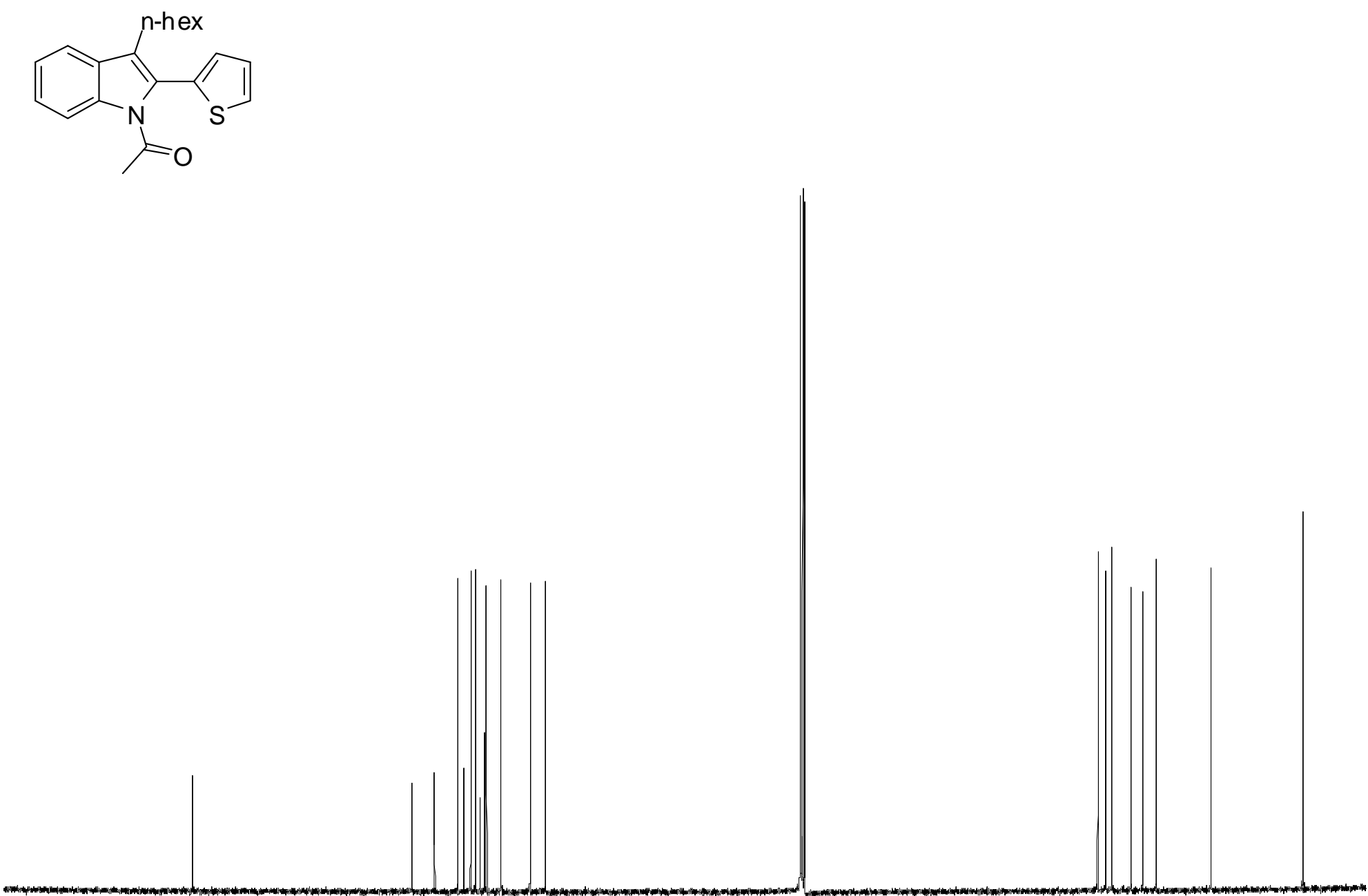

|11111111|1111|1111|1111|111|1111|1111|1111|1111|1111|111|1111|1111|1111|1111|1111|1111|1111|1111|1111 $\begin{array}{llllllllllllllllllll}190 & 180 & 170 & 160 & 150 & 140 & 130 & 120 & 110 & 100 & 90 & 80 & 70 & 60 & 50 & 40 & 30 & 20 & 10 & 0\end{array}$ 

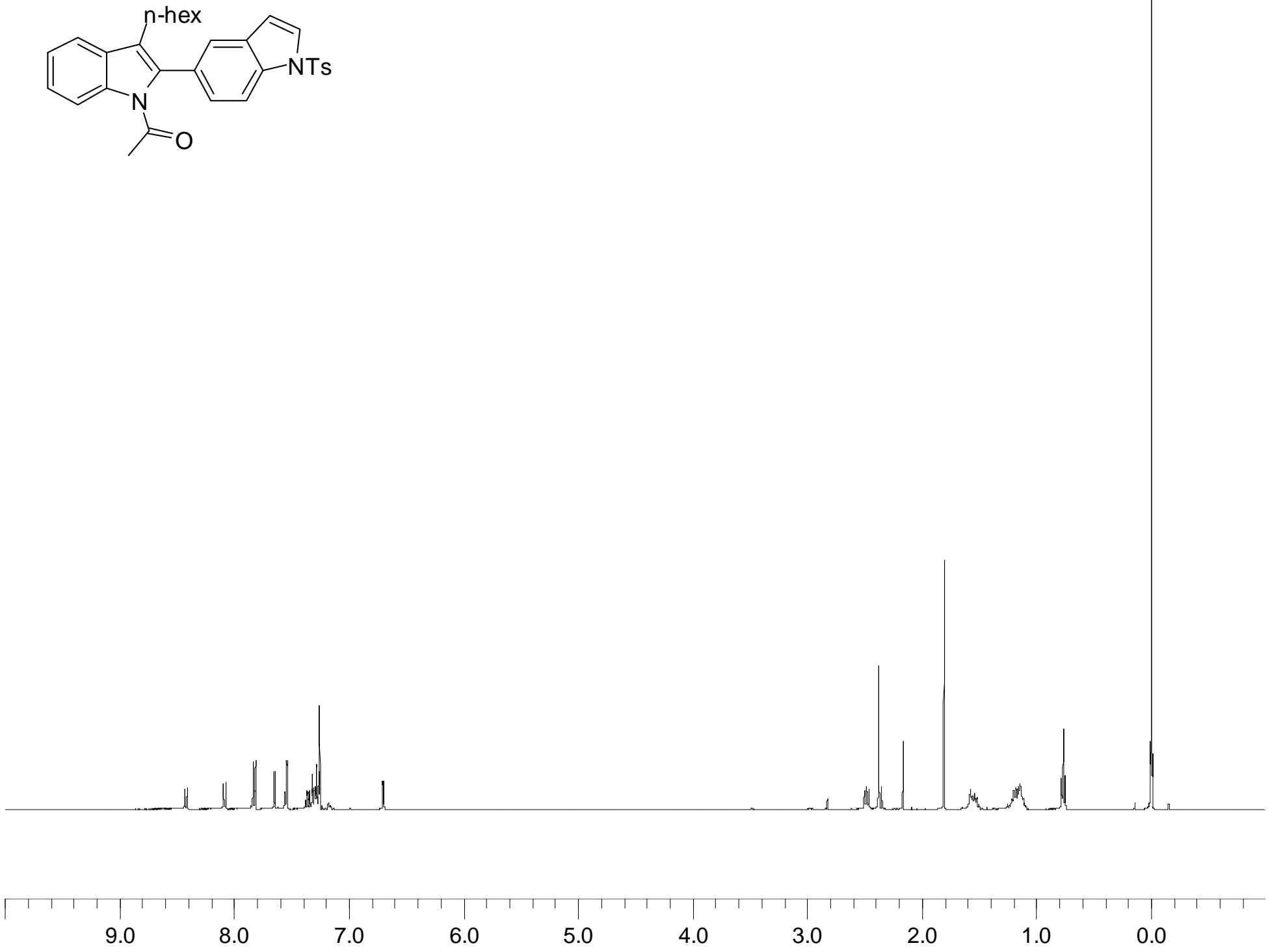

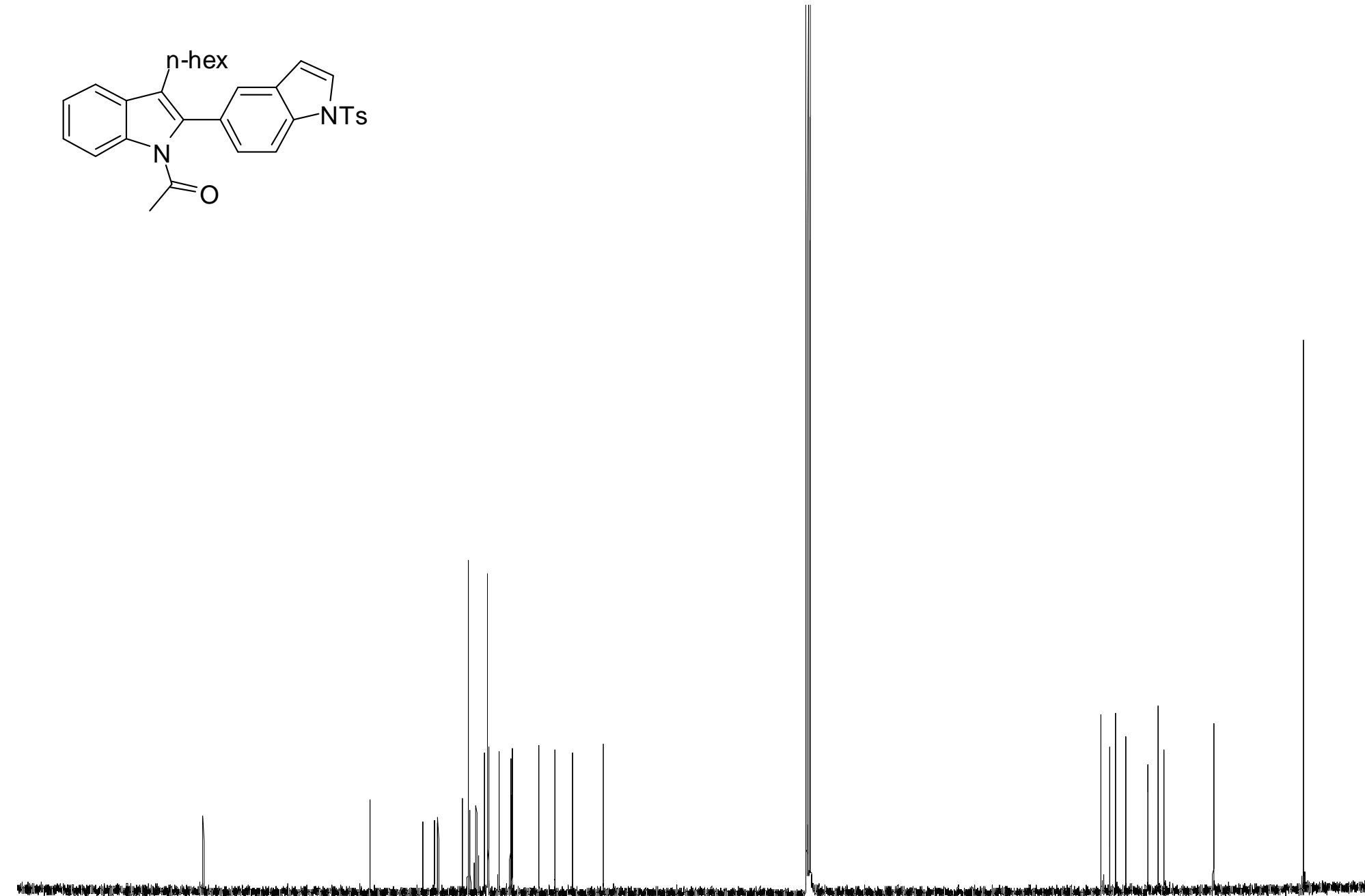

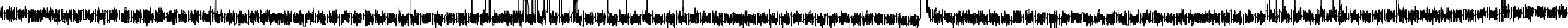

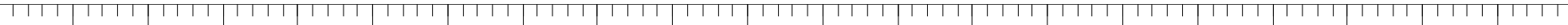

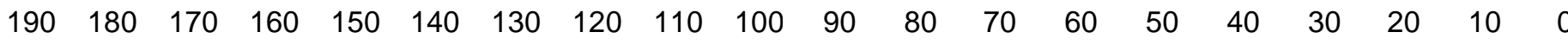



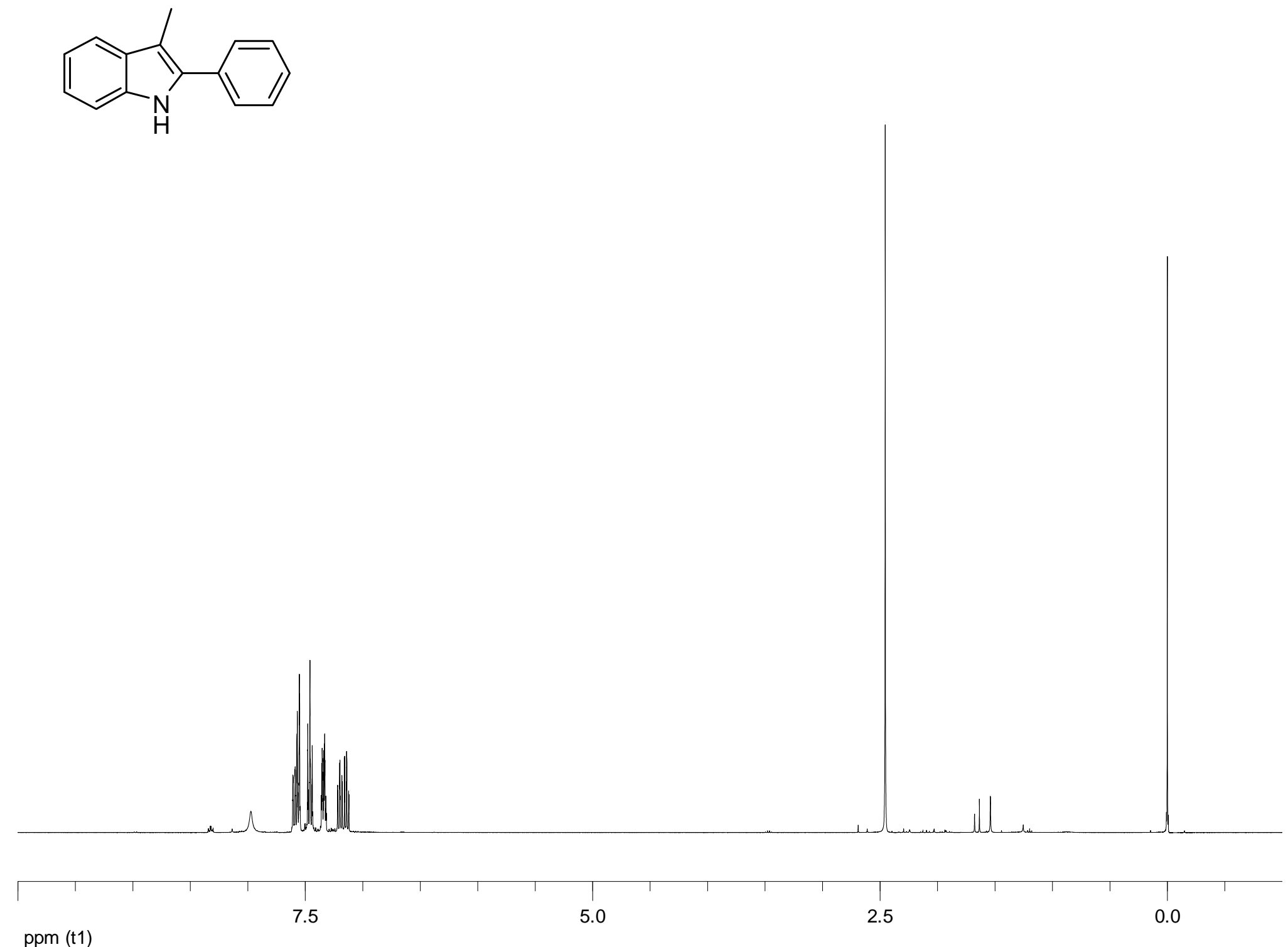

ppm (t1) 

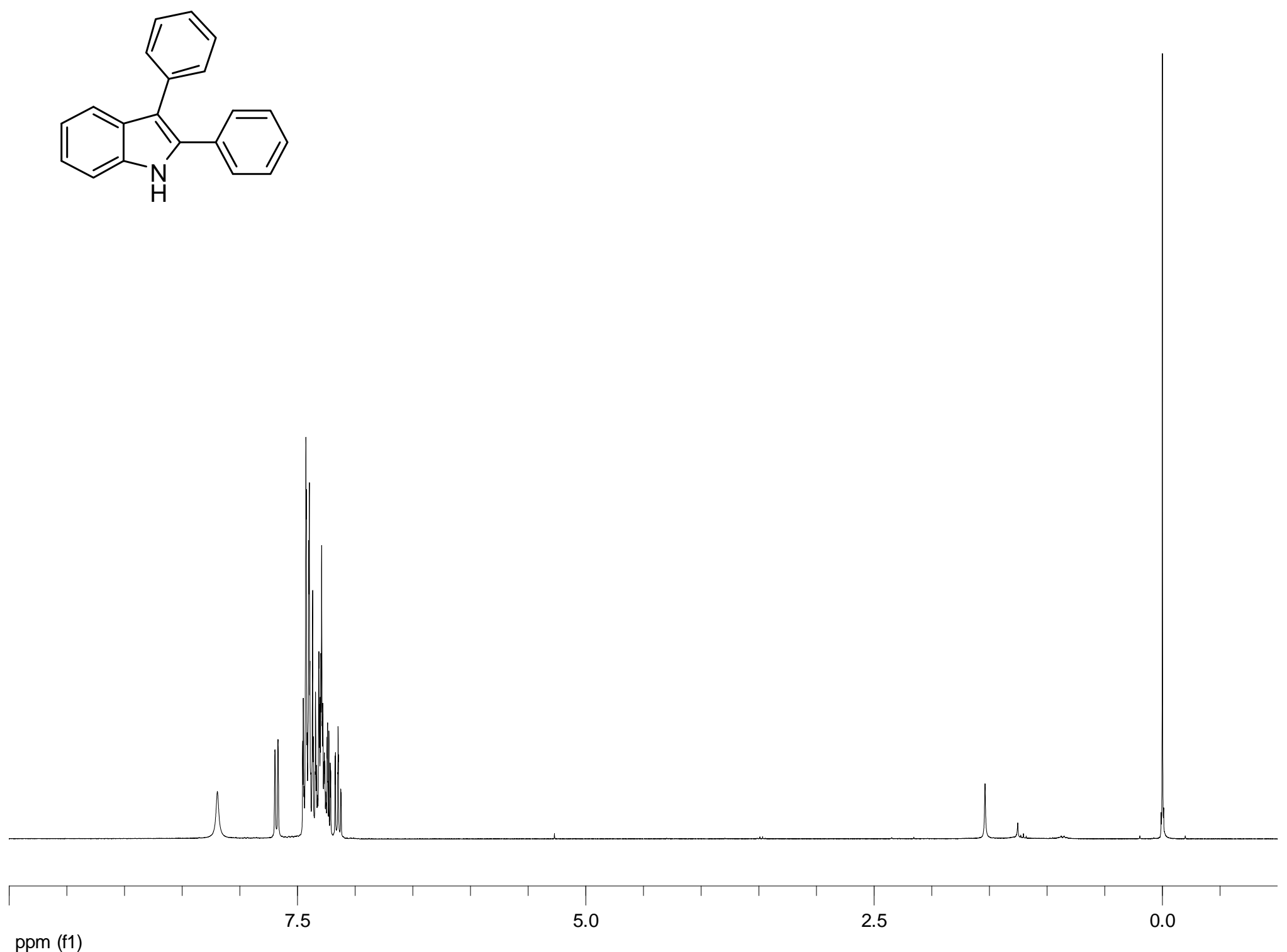

ppm (f1) 

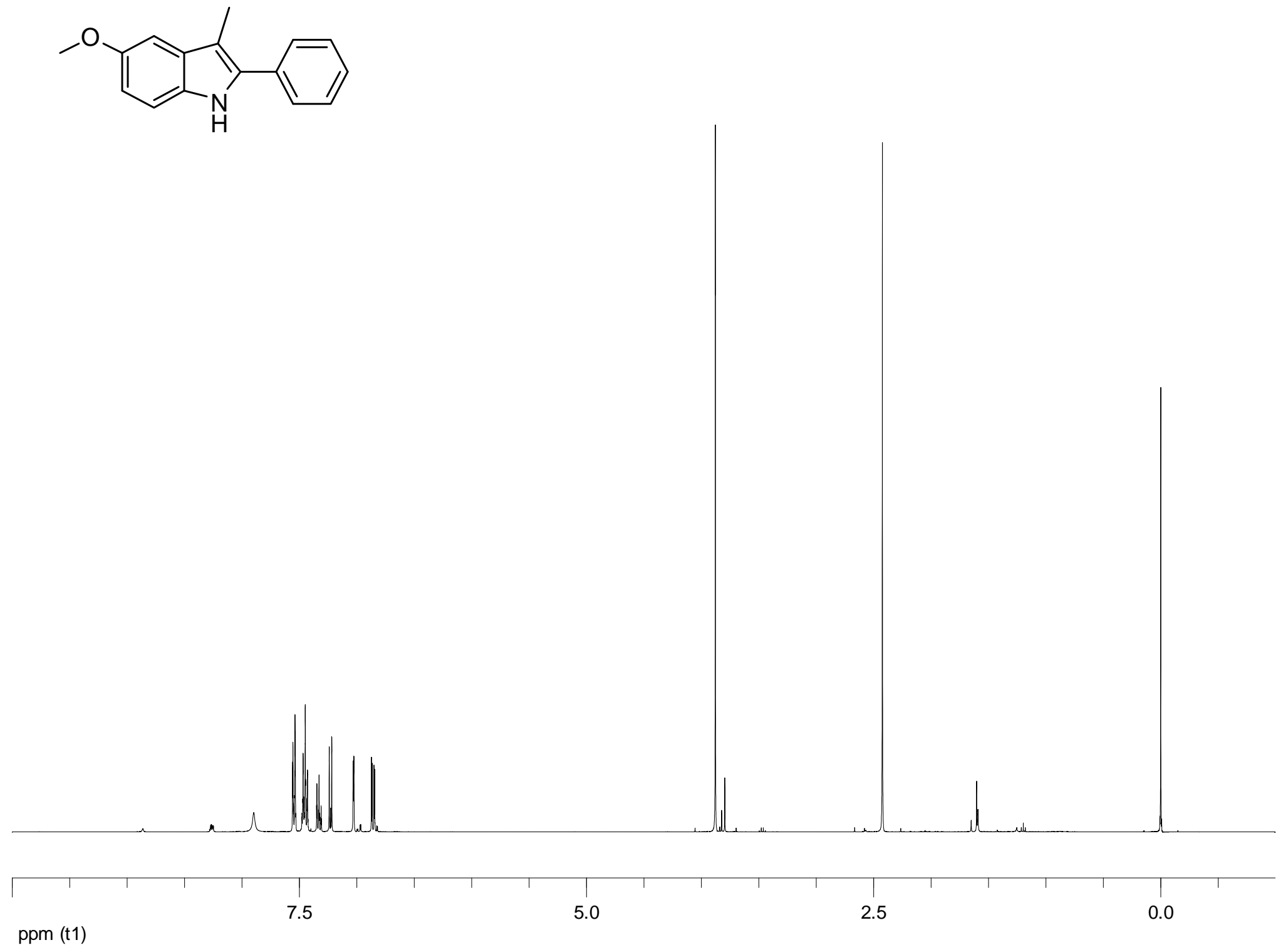

ppm (t1) 

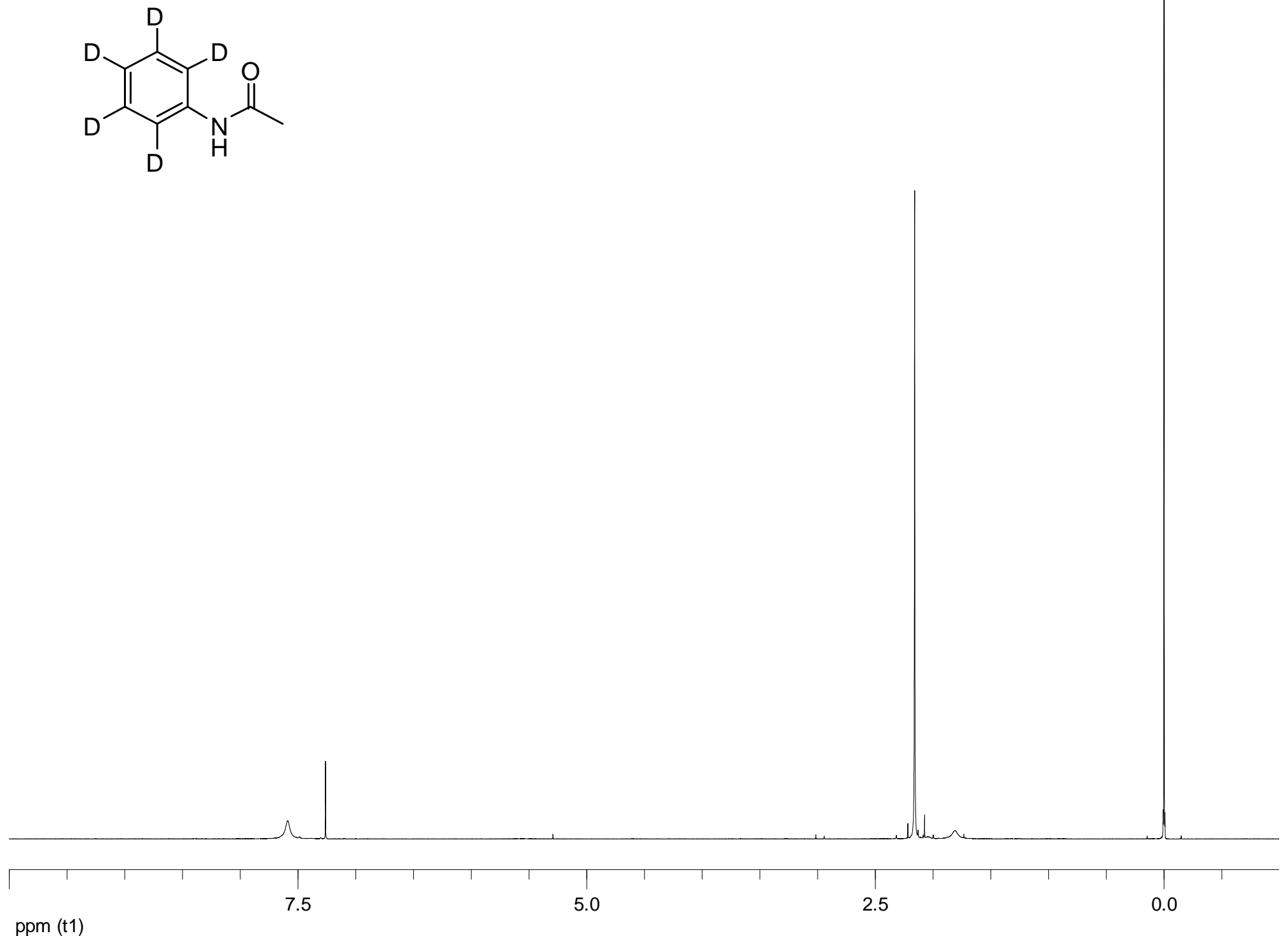

ppm (t1) 
${ }^{1}$ Ikawa, T.; Barder, T. E.; Biscoe, M. R., Buchwald, S. L. J. Am. Chem. Soc. 2007, 43, 13001

${ }^{2}$ Hadjeri, M.; Mariotte, A.-M.; Boumendjel, A. J. Chem. Res. (S), 2002, 9, 463

${ }^{3}$ Guan, J. T.; Weng, T. Q.; Yu, G.-A.; Liu, S. H. Tetrahedron Lett. 2007, 48, 7129-7133

${ }^{4}$ Fresneda, P. M.; Molina, P.; Bleda, J. A. Tetrahedron 2001, 57, 2355-2363

${ }^{5}$ Larock, R. C.; Yum, E. K.; Refvik, M. D. J. Org. Chem. 1998, 63, 7652

${ }^{6}$ Jones, C.P.; Anderson, K.W.; Buchwald, S.L. J. Org. Chem. 2007, 72, 7968

${ }^{7}$ Banerjee, S.; Barnea, E.; Odom, A.L. Organometallics. 2008, 27, 1005

${ }^{8}$ Swenton, J.S.; Shih, C.; Chen, C.-P.; Chou, C.-T. J. Org. Chem. 1990, 55, 2019 